\title{
Three-Component One-Pot Synthesis of Highly Functionalized Bis-indole Derivatives ${ }^{\dagger}$
}

Xue-Bing Chen, ${ }^{\dagger *}$ Sheng-Li Xiong, ${ }^{\dagger}$ Zhi-Xu Xie, ${ }^{\dagger}$ Yong-Chao Wang ${ }^{*}$ and Wei Liu ${ }^{\dagger *}$

$\uparrow$ Key Laboratory of Natural Pharmaceutical and Chemical Biology of Yunnan Province

School of Science Honghe University, Mengzi, Yunnan 661199 (P. R. China)

$\$$ School of Vocational and Technical Education, Yunnan Normal University, Kunming, 650092, P. R. China.

\section{Table of Contents}

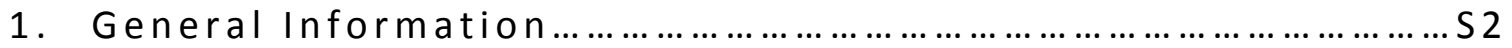

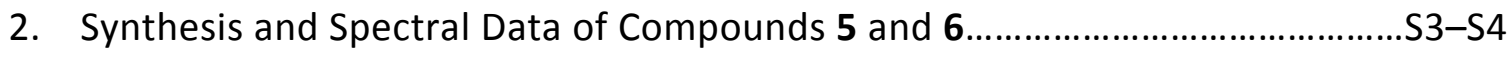

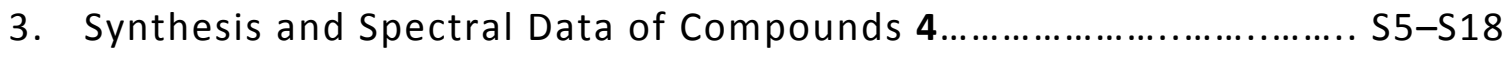

4. Copies of Original ${ }^{1} \mathrm{H}$ and ${ }^{13} \mathrm{C}$ NMR Spectra.............................S19-S70

5. Copies of Original HMRS spectra for representative compound 4..............S71-S77 


\section{General Information}

All chemicals and solvents were directly used, without further purification unless otherwise stated. Materials 2 and 3 were ordered from Adamas Reagent Ltd. The silica gel (200-300 mesh) was used to perform column chromatography. All synthesized chemical molecules were characterized by full spectroscopic data. A Bruker Avance III $400 \mathrm{MHz}$ (1H NMR: $400 \mathrm{MHz}, 13 \mathrm{C}$ NMR: $100 \mathrm{MHz}$ ) was used to characterize. The ${ }^{1} \mathrm{H}$ and ${ }^{13} \mathrm{C}$ nuclear magnetic resonance (NMR) spectra with DMSO-d6 as a solvent and TMS as the internal standard. $\mathrm{J}$ values are given in $\mathrm{Hz}$ and chemical shifts $(\delta)$ are expressed in ppm. A FT-IR Thermo Nicolet Avatar 360 was used to characterize IR spectra by using a $\mathrm{KBr}$ pellet. The whole process of each reaction was monitored in real time by the thin-layer chromatography (TLC) with silica gel GF254. A XT-4A melting point apparatus was used to detect the melting points without further correction. HRMS was characterized on an Agilent LC/Msd TOF and monoisotopic mMass instrument..

All chemicals and solvents were used as received without further purification unless otherwise stated. Column chromatography was performed on silica gel (200-300 mesh). 


\section{Synthesis and Spectral Data of Intermediate 5 and 6}

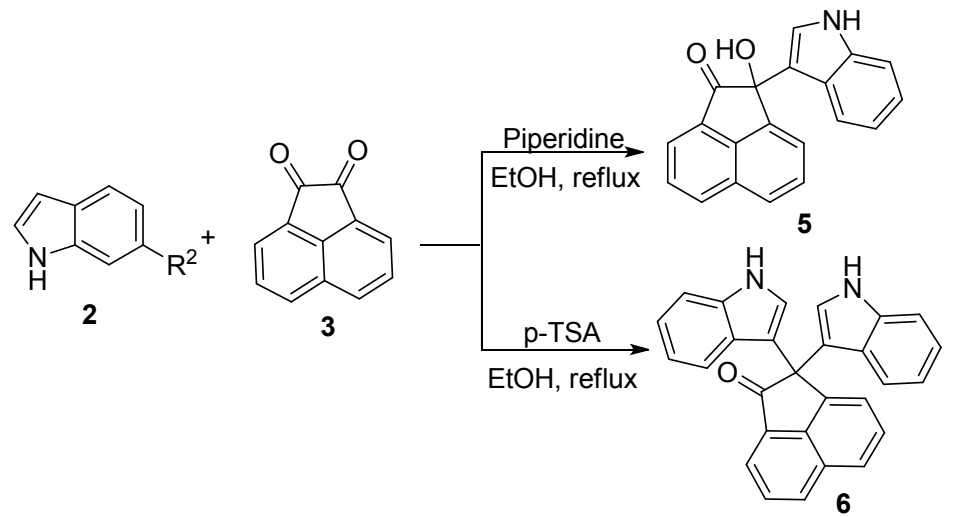

A mixture of indoles $2(1.0 \mathrm{mmol})$, acenaphthylene-1,2-dione $3(1.0 \mathrm{mmol})$, and piperidine $(0.2 \mathrm{mmol})$ was dissolved in ethanol $(15 \mathrm{~mL})$. The reaction mixture was refluxed for a certain period of time as indicated by TLC. Upon completion, as monitored by TLC, the reaction mixture was cooled to room temperature and extracted with $\mathrm{CH}_{2} \mathrm{Cl}_{2}(50 \mathrm{~mL} \times 2)$. The organic phase was dried over anhydrous $\mathrm{Na}_{2} \mathrm{SO}_{4}$ and concentrated under a vacuum. Lastly, the residue was purified by flash chromatography with petroleum ether-ethyl acetate $(1: 1, v / v)$, giving a yellow pure solid 5 .

A mixture of indoles $2(1.0 \mathrm{mmol})$, acenaphthylene-1,2-dione $3(1.0 \mathrm{mmol})$, and p-TSA $(0.2 \mathrm{mmol})$ was dissolved in ethanol $(15 \mathrm{~mL})$. The reaction mixture was refluxed for a certain period of time as indicated by TLC. Upon completion, as monitored by TLC, the reaction mixture was cooled to room temperature and extracted with EtOAc $(50 \mathrm{~mL} \times 2)$. The organic phase was dried over anhydrous $\mathrm{Na}_{2} \mathrm{SO}_{4}$ and concentrated under a vacuum. Lastly, the residue was purified by 
flash chromatography with petroleum ether-ethyl acetate $(1: 1, v / v)$, giving a yellow pure solid 6 .

\section{Spectroscopic data of intermadiate 5 and 6}

\section{2-hydroxy-2-(1H-indol-3-yl)acenaphthylen-1(2H)-one (5)}

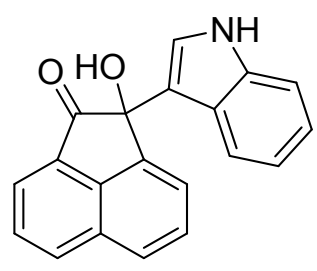

White solid; ${ }^{1} \mathrm{H}$ NMR (400 MHz, DMSO- $d_{6}$ ): $\delta=11.01$ (br, $1 \mathrm{H}, \mathrm{NH}$ ), $8.34(\mathrm{~d}, J=7.6 \mathrm{~Hz}, 1 \mathrm{H}, \mathrm{ArH}), 8.05(\mathrm{~d}, J=7.6 \mathrm{~Hz}, 1 \mathrm{H}, \mathrm{ArH}), 7.95(\mathrm{~d}$, $J=6.8 \mathrm{~Hz}, 1 \mathrm{H}, \mathrm{ArH}), 7.86(\mathrm{t}, J=6.8 \mathrm{~Hz}, 1 \mathrm{H}, \mathrm{ArH}), 7.76(\mathrm{dd}, J=$ 12.0, $6.8 \mathrm{~Hz}, 1 \mathrm{H}, \mathrm{ArH}), 7.66(\mathrm{~d}, J=6.4 \mathrm{~Hz}, 1 \mathrm{H}, \operatorname{ArH}), 7.33$ (d, $J=$ $8.0 \mathrm{~Hz}, 1 \mathrm{H}, \mathrm{ArH}), 7.13(\mathrm{~d}, J=8.0 \mathrm{~Hz}, 1 \mathrm{H}, \mathrm{ArH}), 7.01-7.05$ (m, 2H, ArH), 6.80 (d, $J=$ $8.0 \mathrm{~Hz}, 1 \mathrm{H}, \mathrm{ArH}), 6.79$ (br, $1 \mathrm{H}, \mathrm{OH}) ;{ }^{13} \mathrm{C}$ NMR (100 MHz, DMSO- $\left.d_{6}\right): \delta=203.9,143.2$, $140.6,137.3,132.3,131.0,130.7,129.5,129.2,125.6,125.4,124.3,122.1,121.6,121.5$, 120.9, 119.0, 116.1, 112.0, 79.3; HRMS (ESI-TOF): $\mathrm{m} / z$ calcd for $\mathrm{C}_{20} \mathrm{H}_{13} \mathrm{NnaO}_{2}{ }^{+}$ $\left[(\mathrm{M}+\mathrm{H})^{+}\right], 322.0838$; found, 322.0835 .

\section{2,2-di(1H-indol-3-yl)acenaphthylen-1(2H)-one (6)}

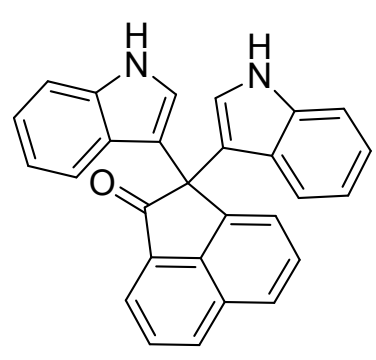

Yellow solid; Mp 290-291 ${ }^{\circ} \mathrm{C}$; ${ }^{1} \mathrm{H}$ NMR (400 MHz, DMSO- $d_{6}$ ): $\delta=11.01(\mathrm{br}, 2 \mathrm{H}, \mathrm{NH}), 8.34(\mathrm{~d}, J=8.0 \mathrm{~Hz}, 1 \mathrm{H}, \mathrm{ArH}), 8.00$ (t, $J=$ $8.0 \mathrm{~Hz}, 2 \mathrm{H}, \mathrm{ArH}), 7.88(\mathrm{t}, J=8.0 \mathrm{~Hz}, 1 \mathrm{H}, \mathrm{ArH}), 7.68(\mathrm{t}, J=8.0$ $\mathrm{Hz}, 1 \mathrm{H}, \mathrm{ArH}), 7.56$ (d, $J=8.0 \mathrm{~Hz}, 1 \mathrm{H}, \mathrm{ArH}), 7.37$ (d, $J=8.0 \mathrm{~Hz}$, 2H, ArH), 6.99-7.05 (m, 4H, ArH), 6.88 (s, 2H, ArH), 6.75 (t, $J=$ $8.0 \mathrm{~Hz}, 2 \mathrm{H}, \mathrm{ArH}) ;{ }^{13} \mathrm{C}$ NMR (100 MHz, DMSO- $\left.d_{6}\right): \delta=203.2,144.3,140.1,137.5,132.5$, 
$132.0,130.9,129.5,129.3,126.3,125.2,124.6,122.7,122.2,121.5,121.1,118.9,115.3$, 112.2, 58.2.

\section{Synthesis and Spectral Data of Compounds 4}

\section{General procedure for the synthesis of bis-indoles 4}

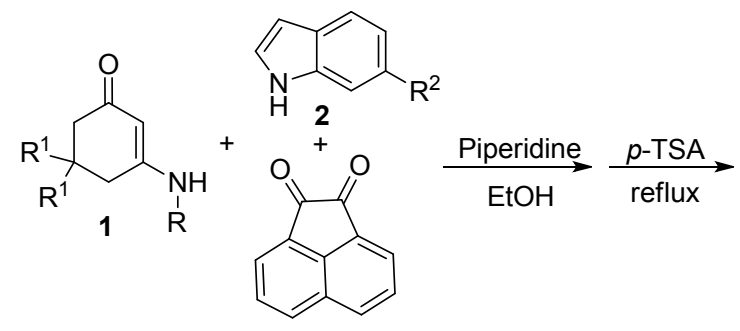

3

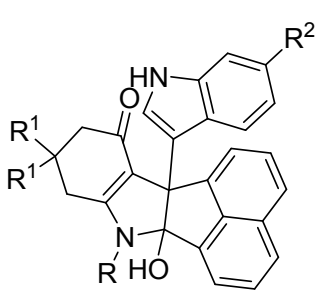

4

The chemical of indoles $2(1.0 \mathrm{mmol})$, acenaphthylene-1,2-dione 3 (1.0 $\mathrm{mmol})$, and piperidine $(0.2 \mathrm{mmol})$ were firstly dissolved in ethanol $(15 \mathrm{~mL})$ together. The reaction mixture was then refluxed for a certain period of time by monitoring with TLC. Enaminone $1(1.0 \mathrm{mmol})$ and p-TSA $(0.5 \mathrm{mmol})$ were further added into the mixture and stirred at reflux temperature. At the end of the reaction, as monitored by TLC, the reaction mixture was terminated by diluted with $50 \mathrm{~mL}$ of water and cooled to room temperature. Afterwards, the mixture was extracted with $50 \mathrm{~mL}$ of EtOAc for two times. Then the organic phase was 
dried over anhydrous $\mathrm{Na}_{2} \mathrm{SO}_{4}$ and concentrated under a vacuum. Finally, flash chromatography with petroleum ether-ethyl acetate $(1: 2, v / v)$ was used to the purification of the residue and giving a white compound solid 4 with high purification.

\section{Spectroscopic data of bis-indoles 4}

7-(4-fluorophenyl)-6b-hydroxy-11b-(1H-indol-3-yl)-8,9,10,11b-tetrahydro-6b $H$-acen aphtho[1,2-b]indol-11(7H)-one (4a)

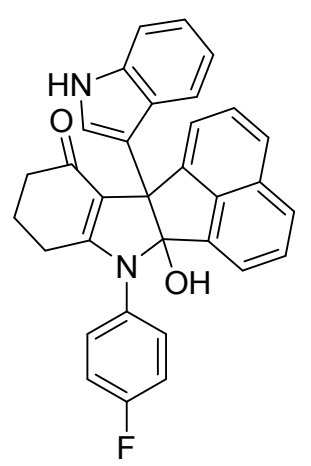

White solid; Mp 221-223 ${ }^{\circ} \mathrm{C}$; IR (KBr): 3477, 3061, 1591, 1553, 1222, 1157, 1024, $788 \mathrm{~cm}^{-1}$; ${ }^{1} \mathrm{H}$ NMR (400 MHz, DMSO- $\left.d_{6}\right): \delta=$ 10.89 (br, 1H, NH), 7.80 (d, $J=8.0 \mathrm{~Hz}, 1 \mathrm{H}, \operatorname{ArH}), 7.73$ (d, $J=8.0$ $\mathrm{Hz}, 1 \mathrm{H}, \mathrm{ArH}), 7.56-7.58$ (m, 1H, ArH), 7.50 (s, 1H, ArH), 7.38 (t, $J$ $=8.0 \mathrm{~Hz}, 1 \mathrm{H}, \mathrm{ArH}), 7.30-7.50(\mathrm{~m}, 5 \mathrm{H}, \mathrm{ArH}), 6.85-6.93(\mathrm{~m}, 2 \mathrm{H}$, ArH), 6.44 (d, $J=8.0 \mathrm{~Hz}, 1 \mathrm{H}, \mathrm{ArH}), 6.26(\mathrm{~s}, 1 \mathrm{H}, \mathrm{ArH}), 5.64$ (br, $1 \mathrm{H}$, $\mathrm{OH}), 2.09-2.20\left(\mathrm{~m}, 1 \mathrm{H}, \mathrm{CH}_{2}\right), 1.85-2.00\left(\mathrm{~m}, 3 \mathrm{H}, \mathrm{CH}_{2}\right) ;{ }^{13} \mathrm{C} \mathrm{NMR}$ $\left(100 \mathrm{MHz}, \mathrm{DMSO}-d_{6}\right): \delta=189.9,162.9,161.7\left(\mathrm{~d},{ }^{1} J_{\mathrm{C}-\mathrm{F}}=244.0 \mathrm{~Hz}\right), 141.7,137.3$, 136.3, 133.6, $132.1\left(\mathrm{~d},{ }^{3} J_{\mathrm{C}-\mathrm{F}}=9.0 \mathrm{~Hz}\right), 131.9\left(\mathrm{~d},{ }^{3} J_{\mathrm{C}-\mathrm{F}}=9.0 \mathrm{~Hz}\right), 131.2,128.9,127.7$, $125.3,123.0,122.4,120.8,120.4,119.9,118.2,116.2\left(\mathrm{~d},{ }^{2} J_{\mathrm{C}-\mathrm{F}}=22.0 \mathrm{~Hz}\right), 116.0\left(\mathrm{~d},{ }^{2} J\right.$ $\left.{ }_{\mathrm{C}-\mathrm{F}}=22.0 \mathrm{~Hz}\right), 111.6,106.2,64.3,37.5,24.3,22.6$; HRMS (ESI-TOF): $\mathrm{m} / \mathrm{z}$ calcd for $\mathrm{C}_{34} \mathrm{H}_{24} \mathrm{FN}_{2} \mathrm{O}_{2}{ }^{+}\left[(\mathrm{M}+\mathrm{H})^{+}\right], 487.1816$; found, 487.1814 .

7-(4-chlorophenyl)-11b-(6-fluoro-1 $H$-indol-3-yl)-6b-hydroxy-8,9,10,11b-tetrahydro6bH-acenaphtho[1,2-b]indol-11(7H)-one (4b) 


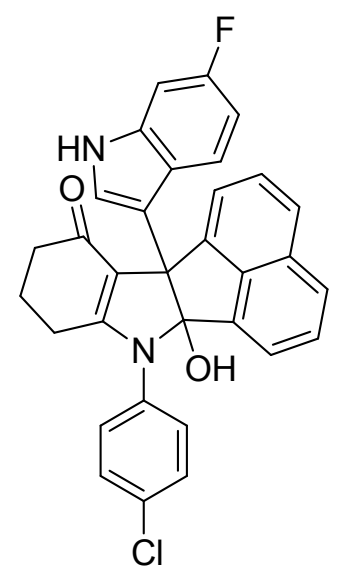

White solid; $\mathrm{Mp}>300{ }^{\circ} \mathrm{C}$; IR (KBr): 3472, 2959, 1559, 1400, 1322, $1140,1092,789 \mathrm{~cm}^{-1} ;{ }^{1} \mathrm{H}$ NMR (400 MHz, DMSO- $\left.d_{6}\right): \delta=10.98(\mathrm{br}$, 1H, NH), 7.80 (d, $J=8.0 \mathrm{~Hz}, 1 \mathrm{H}, \operatorname{ArH}), 7.73(\mathrm{~d}, J=8.0 \mathrm{~Hz}, 1 \mathrm{H}$, ArH), 7.62-7.72 (m, 1H, ArH), $7.50(\mathrm{~d}, J=8.0 \mathrm{~Hz}, 3 \mathrm{H}, \operatorname{ArH})$, 7.40-7.49 (m, 2H, ArH), 7.39 (t, $J=8.0 \mathrm{~Hz}, 1 \mathrm{H}, \mathrm{ArH}), 7.28$ (d, $J=$ $8.0 \mathrm{~Hz}, 1 \mathrm{H}, \mathrm{ArH}), 7.07$ (d, $J=8.0 \mathrm{~Hz}, 3 \mathrm{H}, \mathrm{ArH}), 6.49$ (d, $J=8.0 \mathrm{~Hz}$, 1H, ArH), 6.44 (s, 1H, ArH), 6.22-6.30 (m, 1H, ArH), 5.49 (br, 1H, $\mathrm{OH}), 2.34-2.46\left(\mathrm{~m}, 1 \mathrm{H}, \mathrm{CH}_{2}\right), 2.08-2.20\left(\mathrm{~m}, 4 \mathrm{H}, \mathrm{CH}_{2}\right), 1.94-1.97$ $\left(\mathrm{m}, 1 \mathrm{H}, \mathrm{CH}_{2}\right) ;{ }^{13} \mathrm{C}$ NMR $\left(100 \mathrm{MHz}, \mathrm{DMSO}-d_{6}\right): \delta=190.2,163.1,158.6\left(\mathrm{~d},{ }^{1} J_{\mathrm{C}-\mathrm{F}}=\right.$ $233.0 \mathrm{~Hz}), 146.4,141.7,137.1\left(\mathrm{~d},{ }^{3} J_{\mathrm{C}-\mathrm{F}}=12.0 \mathrm{~Hz}\right), 136.4,136.2,132.5,131.4,131.2$, 129.4, 128.9, 127.8, 125.4, 123.2, 122.4, 119.9, 106.3, $97.5\left(\mathrm{~d},{ }^{2} J_{\mathrm{C}-\mathrm{F}}=25.0 \mathrm{~Hz}\right), 97.3(\mathrm{~d}$, ${ }^{2} J_{\mathrm{C}-\mathrm{F}}=25.0 \mathrm{~Hz}$ ), 64.3, 37.5, 24.3, 22.7; HRMS (ESI-TOF): $\mathrm{m} / \mathrm{z}$ calcd for $\mathrm{C}_{32} \mathrm{H}_{23} \mathrm{ClFN}_{2} \mathrm{O}_{2}^{+}\left[(\mathrm{M}+\mathrm{H})^{+}\right], 521.1427$; found, 521.1427.

11b-(6-chloro-1 $H$-indol-3-yl)-7-(3-chlorophenyl)-6b-hydroxy-8,9,10,11b-tetrahydro6bH-acenaphtho[1,2-b]indol-11(7H)-one $(4 \mathrm{c})$

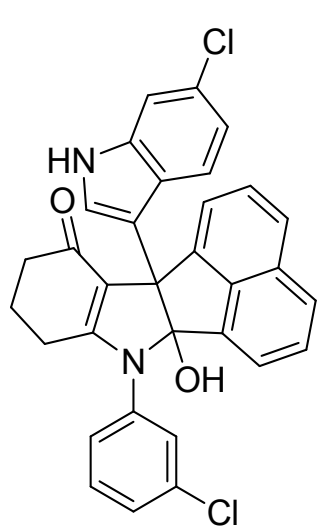

White solid; $\mathrm{Mp}>300^{\circ} \mathrm{C}$; IR (KBr): 3436, 2952, 1589, 1550, 1479, 1194, 1024, $798 \mathrm{~cm}^{-1} ;{ }^{1} \mathrm{H}$ NMR (400 MHz, DMSO- $\left.d_{6}\right): \delta=11.06$ (br, 1H, NH), 7.81 (d, $J=8.4 \mathrm{~Hz}, 1 \mathrm{H}, \mathrm{ArH}), 7.45(\mathrm{~d}, J=8.0 \mathrm{~Hz}, 1 \mathrm{H}$, ArH), 7.62-7.68 (m, 1H, ArH), 7.35-7.55 (m, 7H, ArH), 7.16 (d, $J=$ 7.6 Hz, 2H, ArH), 6.45-6.52 (m, 3H, ArH), 5.55 (br, 1H, OH), 2.46-2.54 (m, 1H, $\left.\mathrm{CH}_{2}\right), 2.09-2.17\left(\mathrm{~m}, 3 \mathrm{H}, \mathrm{CH}_{2}\right), 1.94-2.00(\mathrm{~m}, 1 \mathrm{H}$, $\left.\mathrm{CH}_{2}\right), 1.86-1.89\left(\mathrm{~m}, 1 \mathrm{H}, \mathrm{CH}_{2}\right) ;{ }^{13} \mathrm{C}$ NMR (100 MHz, DMSO- $\left.d_{6}\right): \delta=$ $190.4,163.1,146.5,138.9,137.7,136.2,133.5,131.2,130.8,129.5,128.9,128.4,128.0$, $127.8,125.6,125.3,123.2,122.4,119.8,118.6,111.2,106.4,64.3,37.5,24.3,22.7$; HRMS (ESI-TOF): $m / z$ calcd for $\mathrm{C}_{32} \mathrm{H}_{23} \mathrm{Cl}_{2} \mathrm{~N}_{2} \mathrm{O}_{2}{ }^{+}\left[(\mathrm{M}+\mathrm{H})^{+}\right], 537.1131$; found, 537.1133.

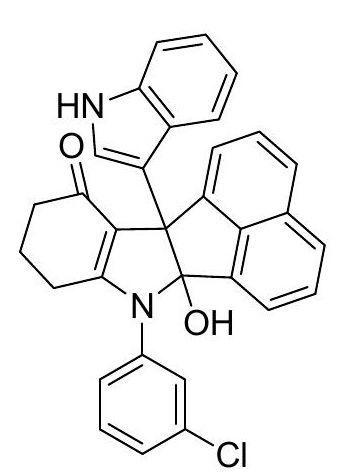

7-(3-chlorophenyl)-6b-hydroxy-11b-(1H-indol-3-yl)-8,9,10,11b-te trahydro-6bH-acenaphtho[1,2-b]indol-11(7H)-one (4d)

White solid; Mp 244-246 ${ }^{\circ} \mathrm{C}$; IR (KBr): 3210, 2950, 1589, 1553, 
1423, 1185, 1018, $789 \mathrm{~cm}^{-1} ;{ }^{1} \mathrm{H}$ NMR (400 MHz, DMSO- $\left.d_{6}\right): \delta=10.92(\mathrm{br}, 1 \mathrm{H}, \mathrm{NH})$, $7.80(\mathrm{~d}, J=8.0 \mathrm{~Hz}, 1 \mathrm{H}, \mathrm{ArH}), 7.73(\mathrm{~d}, J=8.0 \mathrm{~Hz}, 1 \mathrm{H}, \mathrm{ArH}), 7.63-7.66(\mathrm{~m}, 1 \mathrm{H}, \mathrm{ArH})$, 7.50-7.62 (m, 4H, ArH), 7.42 (t, $J=8.0 \mathrm{~Hz}, 1 \mathrm{H}, \mathrm{ArH}), 7.36$ (d, $J=8.0 \mathrm{~Hz}, 1 \mathrm{H}, \mathrm{ArH})$, $7.30(\mathrm{~d}, J=8.0 \mathrm{~Hz}, 1 \mathrm{H}, \mathrm{ArH}), 7.16(\mathrm{~d}, J=6.4 \mathrm{~Hz}, 1 \mathrm{H}, \mathrm{ArH}), 6.49$ (d, $J=7.2 \mathrm{~Hz}, 1 \mathrm{H}$, ArH), 6.39 (s, 1H, ArH), 6.37-6.49 (m, 1H, ArH), 5.59 (br, 1H, OH), 2.44-2.47 (m, 1H, $\left.\mathrm{CH}_{2}\right), 2.10-2.20\left(\mathrm{~m}, 3 \mathrm{H}, \mathrm{CH}_{2}\right), 1.95-2.00\left(\mathrm{~m}, 1 \mathrm{H}, \mathrm{CH}_{2}\right), 1.85-1.89\left(\mathrm{~m}, 1 \mathrm{H}, \mathrm{CH}_{2}\right)$; ${ }^{13} \mathrm{CNMR}\left(100 \mathrm{MHz}\right.$, DMSO- $\left.d_{6}\right): \delta=189.9,162.8,146.8,141.6,139.0,137.4,136.3$, $133.5,131.2,130.8,129.5,128.9,128.4,127.9,125.5,123.1,122.5,120.4,119.7,118.2$, 111.6, 106.4, 64.5, 37.6, 24.4, 22.7; HRMS(ESI-TOF): $m / z$ calcd for $\mathrm{C}_{32} \mathrm{H}_{24} \mathrm{ClN}_{2} \mathrm{O}_{2}{ }^{+}$ $\left[(\mathrm{M}+\mathrm{H})^{+}\right]$, 503.1521; found, 503.1520 .

\section{7-(2-chlorophenyl)-6b-hydroxy-11b-(1H-indol-3-yl)-8,9,10,11b-tetrahydro-6bH-acen aphtho[1,2-b]indol-11(7H)-one $(4 \mathrm{e})$}

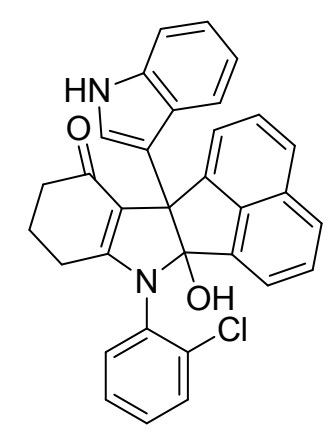

White solid; Mp 242-243 ${ }^{\circ} \mathrm{C}$; IR (KBr): 3438, 2963, 1610, 1562, $1479,1251,1103,780 \mathrm{~cm}^{-1} ;{ }^{1} \mathrm{H}$ NMR (400 MHz, DMSO- $\left.d_{6}\right): \delta=$ 10.89 (br, 1H, NH), 7.88 (d, $J=8.4 \mathrm{~Hz}, 1 \mathrm{H}, \mathrm{ArH}), 7.80$ (d, $J=8.0$ $\mathrm{Hz}, 1 \mathrm{H}, \mathrm{ArH}), 7.73$ (d, $J=8.0 \mathrm{~Hz}, 1 \mathrm{H}, \mathrm{ArH}), 7.43-7.56$ (m, 5H, ArH), 7.27-7.35 (m, 2H, ArH), 6.84-6.90 (m, 2H, ArH), 6.34 (d, $J$ $=7.2 \mathrm{~Hz}, 1 \mathrm{H}, \mathrm{ArH}), 6.31(\mathrm{~s}, 1 \mathrm{H}, \mathrm{ArH}), 5.50(\mathrm{br}, 1 \mathrm{H}, \mathrm{OH})$, 2.08-2.30 (m, 4H, $\left.\mathrm{CH}_{2}\right), 1.94-1.98\left(\mathrm{~m}, 1 \mathrm{H}, \mathrm{CH}_{2}\right), 1.79-1.82(\mathrm{~m}$, $\left.1 \mathrm{H}, \mathrm{CH}_{2}\right) ;{ }^{13} \mathrm{C}$ NMR $\left(100 \mathrm{MHz}, \mathrm{DMSO}-d_{6}\right): \delta=190.3,163.8,147.1,141.2,137.3,136.8$, $134.8,134.3,133.9,131.0,130.6,130.3,128.9,128.4,127.8,125.3,123.0,121.8,120.3$, 119.2, 118.2, 111.5, 106.2, 64.6, 37.7, 24.0, 22.5; HRMS (ESI-TOF): $\mathrm{m} / \mathrm{z}$ calcd for $\mathrm{C}_{32} \mathrm{H}_{24} \mathrm{ClN}_{2} \mathrm{O}_{2}^{+}\left[(\mathrm{M}+\mathrm{H})^{+}\right], 503.1521$; found, 503.1515.

11b-(6-chloro-1H-indol-3-yl)-6b-hydroxy-7-phenyl-8,9,10,11b-tetrahydro-6b $H$-acen aphtho[1,2-b]indol-11(7H)-one (4f)

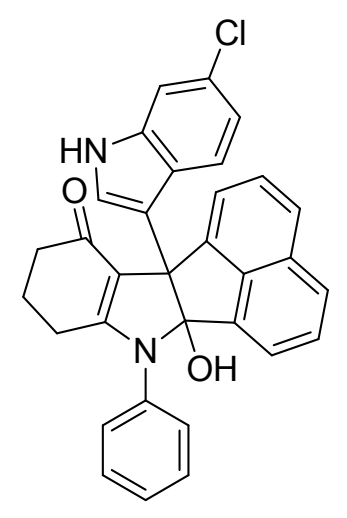

White solid; $\mathrm{Mp}>300{ }^{\circ} \mathrm{C}$; IR (KBr): 3413, 2964, 1620, 1598, 1402, 1187, 1096, $784 \mathrm{~cm}^{-1} ;{ }^{1} \mathrm{H}$ NMR (400 MHz, DMSO- $\left.d_{6}\right): \delta=10.95$ (br, 1H, NH), $7.78(\mathrm{~d}, J=8.4 \mathrm{~Hz}, 1 \mathrm{H}, \operatorname{ArH}), 7.72(\mathrm{~d}, J=8.4 \mathrm{~Hz}, 1 \mathrm{H}$, 
ArH), 7.64-7.74 (m, 1H, ArH), 7.48-7.52 (m, 1H, ArH), 7.39-7.45 (m, 3H, ArH), 7.34 (t, $J=8.0 \mathrm{~Hz}, 2 \mathrm{H}, \mathrm{ArH}), 7.20-7.26$ (m, 2H, ArH), 6.36-6.44 (m, 3H, ArH), 5.59 (br, 1H, $\mathrm{OH}), 2.38-2.49\left(\mathrm{~m}, 1 \mathrm{H}, \mathrm{CH}_{2}\right), 2.08-2.19\left(\mathrm{~m}, 3 \mathrm{H}, \mathrm{CH}_{2}\right), 1.93-1.99\left(\mathrm{~m}, 1 \mathrm{H}, \mathrm{CH}_{2}\right)$, 1.83-1.88 (m, $\left.1 \mathrm{H}, \mathrm{CH}_{2}\right) ;{ }^{13} \mathrm{C}$ NMR (100 MHz, DMSO- $\left.d_{6}\right): \delta=189.4,161.9,147.1,141.7$, 137.2, 136.8, 136.2, 132.4, 132.0, 131.2, 128.9, 127.9, 127.8, 125.4, 123.0, 122.1, 121.1, 120.4, 119.8, 118.1, 111.5, 106.7, 64.2, 51.5, 37.6, 33.9, 30.0, 27.6; HRMS (ESI-TOF): $m / z$ calcd for $\mathrm{C}_{32} \mathrm{H}_{24} \mathrm{ClN}_{2} \mathrm{O}_{2}^{+}\left[(\mathrm{M}+\mathrm{H})^{+}\right], 503.1521$; found, 503.1522.

\section{6b-hydroxy-11b-(1H-indol-3-yl)-7-phenyl-8,9,10,11b-tetrahydro-6bH-acenaphtho[1, 2-b]indol-11(7H)-one $(4 \mathrm{~g})$}

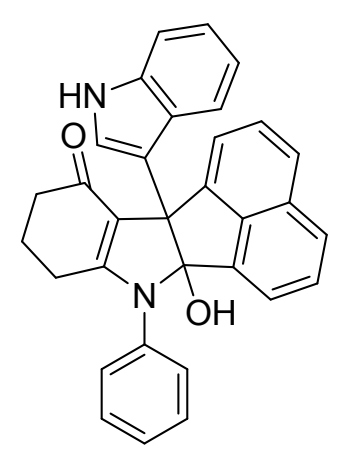

White solid; Mp 276-278 ${ }^{\circ} \mathrm{C}$; IR (KBr): 3489, 2959, 1587, 1548, 1436, 1244, 1132, $739 \mathrm{~cm}^{-1} ;{ }^{1} \mathrm{H}$ NMR (400 MHz, DMSO- $\left.d_{6}\right): \delta=$ $10.88(\mathrm{br}, 1 \mathrm{H}, \mathrm{NH}), 7.79$ (d, $J=8.0 \mathrm{~Hz}, 1 \mathrm{H}, \mathrm{ArH}), 7.73$ (d, $J=8.0$ $\mathrm{Hz}, 1 \mathrm{H}, \mathrm{ArH}), 7.50-7.54(\mathrm{~m}, 1 \mathrm{H}, \mathrm{ArH}), 7.50(\mathrm{~s}, 1 \mathrm{H}, \mathrm{ArH})$, 7.36-7.45 (m, 3H, ArH), 7.23-7.34 (m, 3H, ArH), 6.88 (s, 1H, ArH), $6.41(\mathrm{~d}, J=8.0 \mathrm{~Hz}, 1 \mathrm{H}, \mathrm{ArH}), 6.26-6.40(\mathrm{~m}, 1 \mathrm{H}, \mathrm{ArH}), 6.26$ (s, 1H, ArH), $5.67(\mathrm{br}, 1 \mathrm{H}, \mathrm{OH}), 2.39-2.49\left(\mathrm{~m}, 1 \mathrm{H}, \mathrm{CH}_{2}\right)$, $2.08-2.20\left(\mathrm{~m}, 3 \mathrm{H}, \mathrm{CH}_{2}\right), 1.84-1.98\left(\mathrm{~m}, 2 \mathrm{H}, \mathrm{CH}_{2}\right) ;{ }^{13} \mathrm{C}$ NMR $\left(100 \mathrm{MHz}, \mathrm{DMSO}-d_{6}\right): \delta=$ $189.9,162.4,141.8,137.5,136.3,131.2,129.8,129.3,128.8,128.0,127.6,125.3,123.0$, 122.4, 120.4, 119.9, 118.2, 111.6, 106.3, 64.4, 37.6, 24.5, 22.7; HRMS (ESI-TOF): $\mathrm{m} / \mathrm{z}$ calcd for $\mathrm{C}_{32} \mathrm{H}_{25} \mathrm{~N}_{2} \mathrm{O}_{2}{ }^{+}\left[(\mathrm{M}+\mathrm{H})^{+}\right], 469.1911$; found, 469.1910 .

6b-hydroxy-11b-(1H-indol-3-yl)-7-( $m$-tolyl)-8,9,10,11b-tetrahydro-6bH-acenaphtho[ 1,2-b]indol-11(7H)-one (4h)

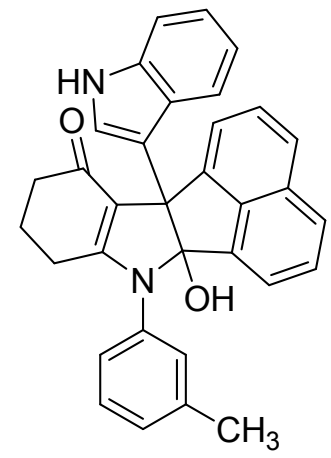

White solid; Mp 270-272 ${ }^{\circ} \mathrm{C}$; IR (KBr): 3427, 1715, 1632, 1586, 1404, 1403, 1191, $785 \mathrm{~cm}^{-1} ;{ }^{1} \mathrm{H}$ NMR (400 MHz, DMSO- $\left.d_{6}\right): \delta=$ $10.88(\mathrm{br}, 1 \mathrm{H}, \mathrm{NH}), 7.78(\mathrm{~d}, J=8.2 \mathrm{~Hz}, 1 \mathrm{H}, \operatorname{ArH}), 7.72(\mathrm{~d}, J=8.0$ Hz, 1H, ArH), 7.60-7.67 (m, 1H, ArH), 7.50 (s, 1H, ArH), 7.35 (t, J 
$=7.8 \mathrm{~Hz}, 1 \mathrm{H}, \operatorname{ArH}), 7.29(\mathrm{~d}, J=8.0 \mathrm{~Hz}, 2 \mathrm{H}, \operatorname{ArH}), 7.20(\mathrm{~d}, J=7.8 \mathrm{~Hz}, 1 \mathrm{H}, \operatorname{ArH})$, 6.87-7.09 (m, 4H, ArH), 6.46 (d, $J=6.8 \mathrm{~Hz}, 1 \mathrm{H}, \mathrm{ArH}), 6.23$ (s, 1H, ArH), 6.39-6.44 (m, 1H, ArH), 5.63 (br, 1H, OH), 2.38-2.45 (m, 1H, $\left.\mathrm{CH}_{2}\right), 2.29$ (s, 3H, $\left.\mathrm{CH}_{3}\right), 2.05-2.20(\mathrm{~m}$, $\left.3 \mathrm{H}, \mathrm{CH}_{2}\right), 1.94-1.98\left(\mathrm{~m}, 1 \mathrm{H}, \mathrm{CH}_{2}\right), 1.83-1.87\left(\mathrm{~m}, 1 \mathrm{H}, \mathrm{CH}_{2}\right) ;{ }^{13} \mathrm{C} \mathrm{NMR}(100 \mathrm{MHz}$, DMSO- $\left.d_{6}\right): \delta=189.9,163.3,147.0,141.9,138.6,137.3,136.3,131.2,130.0,128.8$, 128.6, 127.6, 126.8, 125.3, 123.0, 122.3, 120.4, 120.0, 118.2, 111.6, 106.3, 64.4, 37.6, 24.5, 22.7, 21.4; HRMS (ESI-TOF): $m / z$ calcd for $\mathrm{C}_{33} \mathrm{H}_{27} \mathrm{~N}_{2} \mathrm{O}_{2}{ }^{+}\left[(\mathrm{M}+\mathrm{H})^{+}\right], 483.2067$; found, 483.2069 .

\section{1b-(6-fluoro-1H-indol-3-yl)-6b-hydroxy-7-(4-methoxyphenyl)-8,9,10,11b-tetrahydr o-6bH-acenaphtho[1,2-b]indol-11(7H)-one (4i)}

White solid; $\mathrm{Mp}>300^{\circ} \mathrm{C}$; IR (KBr): 3337, 2953, 1614, 1515, 1402, 1249, 1142, 784

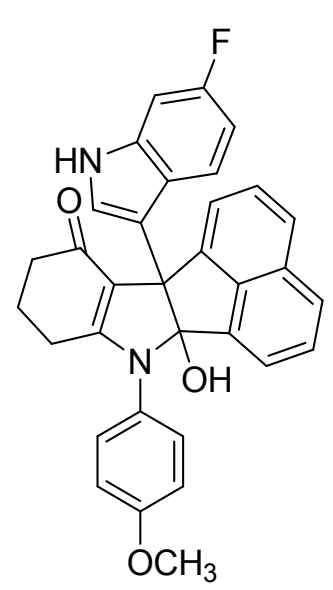

$\mathrm{cm}^{-1} ;{ }^{1} \mathrm{H}$ NMR (400 MHz, DMSO- $\left.d_{6}\right): \delta=10.95$ (br, $\left.1 \mathrm{H}, \mathrm{NH}\right), 7.79$ (d, $J=8.0 \mathrm{~Hz}, 1 \mathrm{H}, \mathrm{ArH}), 7.73$ (d, $J=8.4 \mathrm{~Hz}, 1 \mathrm{H}, \mathrm{ArH}), 7.65-7.70$ (m, 1H, ArH), $7.50(\mathrm{~s}, 1 \mathrm{H}, \mathrm{ArH}), 7.38(\mathrm{t}, J=8.0 \mathrm{~Hz}, 1 \mathrm{H}, \mathrm{ArH})$, 7.10-7.14 (m, 3H, ArH), 7.06 (d, $J=8.0 \mathrm{~Hz}, 1 \mathrm{H}, \mathrm{ArH}), 6.98$ (d, $J=$ $8.0 \mathrm{~Hz}, 2 \mathrm{H}, \mathrm{ArH}), 6.47$ (d, $J=7.8 \mathrm{~Hz}, 1 \mathrm{H}, \mathrm{ArH}), 6.25$ (s, 1H, ArH), 6.24-6.35 (m, 1H, ArH), 5.49 (br, 1H, OH), $3.79\left(\mathrm{~s}, 3 \mathrm{H}, \mathrm{OCH}_{3}\right)$, 2.30-2.37 (m, 1H, $\left.\mathrm{CH}_{2}\right), 2.07-2.18\left(\mathrm{~m}, 3 \mathrm{H}, \mathrm{CH}_{2}\right), 1.91-1.97$ (m, $1 \mathrm{H}$, $\left.\mathrm{CH}_{2}\right), 1.80-1.86\left(\mathrm{~m}, 1 \mathrm{H}, \mathrm{CH}_{2}\right) ;{ }^{13} \mathrm{C}$ NMR (100 MHz, DMSO- $\left.d_{6}\right): \delta$ $=189.6,163.9,159.0,158.6\left(\mathrm{~d},{ }^{1} J_{\mathrm{C}-\mathrm{F}}=232.0 \mathrm{~Hz}\right), 147.0,141.8,137.1\left(\mathrm{~d},{ }^{3} J_{\mathrm{C}-\mathrm{F}}=13.0\right.$ Hz), 136.3, 131.3, 131.2, 129.7, 128.9, 127.7, 125.2, 123.0, 122.3, 120.1, 114.4, 106.2, $97.5\left(\mathrm{~d},{ }^{2} J_{\mathrm{C}-\mathrm{F}}=24.0 \mathrm{~Hz}\right), 97.2\left(\mathrm{~d},{ }^{2} J_{\mathrm{C}-\mathrm{F}}=24.0 \mathrm{~Hz}\right), 64.1,55.8,37.5,24.3,22.6$; HRMS (ESI-TOF): $m / z$ calcd for $\mathrm{C}_{33} \mathrm{H}_{26} \mathrm{FN}_{2} \mathrm{O}_{3}{ }^{+}\left[(\mathrm{M}+\mathrm{H})^{+}\right], 517.1922$; found, 517.1923.

$11 b$-(6-chloro-1H-indol-3-yl)-6b-hydroxy-7-(4-methoxyphenyl)-8,9,10,11b-tetrahydr

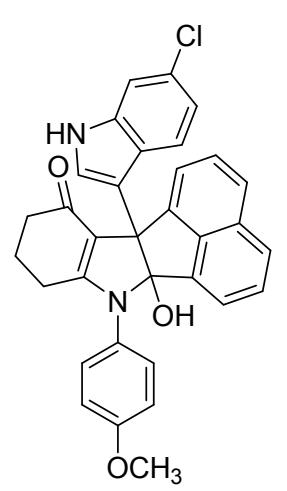
o-6bH-acenaphtho[1,2-b]indol-11(7H)-one $(4 \mathrm{j})$

White solid; $\mathrm{Mp}>300{ }^{\circ} \mathrm{C}$; IR (KBr): 3448, 2938, 1612, 1510, 1399, 1251, 1098, $787 \mathrm{~cm}^{-1} ;{ }^{1} \mathrm{H}$ NMR (400 MHz, DMSO- $\left.d_{6}\right): \delta=11.03(\mathrm{br}$, 
1H, NH), 7.79 (d, $J=8.4 \mathrm{~Hz}, 1 \mathrm{H}, \mathrm{ArH}), 7.73$ (d, $J=8.0 \mathrm{~Hz}, 1 \mathrm{H}, \mathrm{ArH}), 7.60-7.69$ (m, 1H, ArH), 7.49-7.59 (m, 2H, ArH), 7.38 (t, $J=6.8 \mathrm{~Hz}, 2 \mathrm{H}, \operatorname{ArH}), 7.34$ (s, 1H, ArH), 7.10-7.16 (m, 3H, ArH), 6.97 (d, $J=7.6 \mathrm{~Hz}, 1 \mathrm{H}, \mathrm{ArH}), 6.47$ (d, $J=7.2 \mathrm{~Hz}, 1 \mathrm{H}, \operatorname{ArH})$, $6.30(\mathrm{~s}, 1 \mathrm{H}, \mathrm{ArH}), 5.53(\mathrm{br}, 1 \mathrm{H}, \mathrm{OH}), 3.78\left(\mathrm{~s}, 3 \mathrm{H}, \mathrm{OCH}_{3}\right), 2.30-2.38\left(\mathrm{~m}, 1 \mathrm{H}, \mathrm{CH}_{2}\right)$, 2.06-2.18 (m, 3H, CH $), 1.92-1.96\left(\mathrm{~m}, 1 \mathrm{H}, \mathrm{CH}_{2}\right), 1.81-1.86\left(\mathrm{~m}, 1 \mathrm{H}, \mathrm{CH}_{2}\right) ;{ }^{13} \mathrm{C} \mathrm{NMR}$ (100 MHz, DMSO- $\left.d_{6}\right): \delta=189.7,163.9,159.0,146.8,141.8,137.7,136.3,131.3,131.2$, $129.7,128.9,127.8,125.3,123.1,122.2,120.1,118.5,114.4,111.1,106.2,64.0,55.8$, 37.5, 24.3, 22.6; HRMS (ESI-TOF): $m / z$ calcd for $\mathrm{C}_{33} \mathrm{H}_{26} \mathrm{ClN}_{2} \mathrm{O}_{3}{ }^{+}\left[(\mathrm{M}+\mathrm{H})^{+}\right], 533.1626$; found, 533.1641 .

6b-hydroxy-11b-(1H-indol-3-yl)-7-(4-methoxyphenyl)-8,9,10,11b-tetrahydro-6bH-ac enaphtho[1,2-b]indol-11(7H)-one $(4 k)$

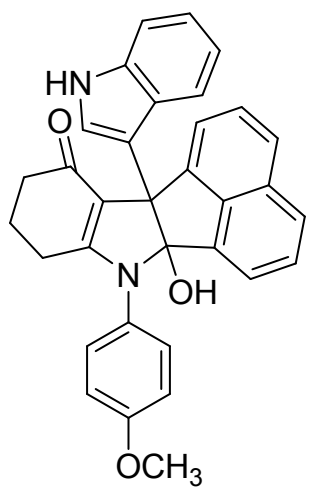

White solid; Mp 280-282 ${ }^{\circ} \mathrm{C}$; IR (KBr): 3329, 2957, 1610, 1507, 1398, 1249, 1101, $785 \mathrm{~cm}^{-1} ;{ }^{1} \mathrm{H}$ NMR (400 MHz, DMSO- $\left.d_{6}\right): \delta=$ 10.89 (br, 1H, NH), 7.79 (d, $J=8.0 \mathrm{~Hz}, 1 \mathrm{H}, \mathrm{ArH}), 7.72$ (d, $J=8.0$ $\mathrm{Hz}, 1 \mathrm{H}, \mathrm{ArH}), 7.49-7.56(\mathrm{~m}, 2 \mathrm{H}, \mathrm{ArH}), 7.38(\mathrm{t}, J=8.0 \mathrm{~Hz}, 1 \mathrm{H}$, ArH), 7.29 (d, $J=8.0 \mathrm{~Hz}, 1 \mathrm{H}, \mathrm{ArH}), 7.04-7.13$ (m, 3H, ArH), 6.98 $(\mathrm{d}, J=8.0 \mathrm{~Hz}, 3 \mathrm{H}, \mathrm{ArH}), 6.59-6.68(\mathrm{~m}, 1 \mathrm{H}, \operatorname{ArH}), 6.47(\mathrm{~d}, J=8.0$ $\mathrm{Hz}, 1 \mathrm{H}, \mathrm{ArH}), 6.17$ (s, 1H, ArH), 5.59 (br, 1H, OH), 3.79 (s, 3H, $\left.\mathrm{OCH}_{3}\right), 2.27-2.37\left(\mathrm{~m}, 1 \mathrm{H}, \mathrm{CH}_{2}\right), 1.81-2.18\left(\mathrm{~m}, 5 \mathrm{H}, \mathrm{CH}_{2}\right) ;{ }^{13} \mathrm{CNMR}(100 \mathrm{MHz}$, DMSO- $\left.d_{6}\right): \delta=189.9,162.6,159.0,142.0,137.3,136.3,131.5,131.2,129.6,128.9$, $128.0,127.7,125.2,122.9,122.0,120.4,120.0,118.0,114.5,111.5,106.6,63.9,55.7$, 51.6, 33.7, 30.0, 27.8; HRMS(ESI-TOF): $m / z$ calcd for $\mathrm{C}_{33} \mathrm{H}_{27} \mathrm{~N}_{2} \mathrm{O}_{3}{ }^{+}\left[(\mathrm{M}+\mathrm{H})^{+}\right], 499.2016$; found, 499.2017.

11b-(6-fluoro-1H-indol-3-yl)-7-(4-fluorophenyl)-6b-hydroxy-9,9-dimethyl-8,9,10,11b -tetrahydro-6bH-acenaphtho[1,2-b]indol-11(7H)-one (4I)

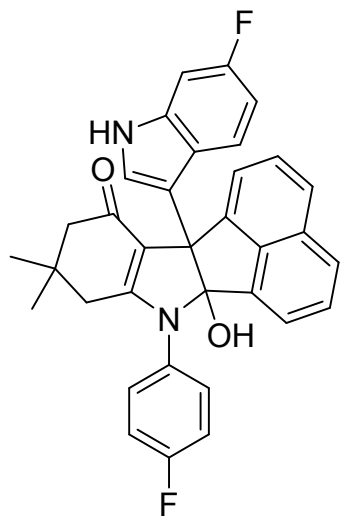

White solid; $\mathrm{Mp} 256-258{ }^{\circ} \mathrm{C}$; IR (KBr): 3339, 2955, 1557, 1491, 1321, 1192, 1092, $787 \mathrm{~cm}^{-1} ;{ }^{1} \mathrm{H}$ NMR (400 MHz, DMSO- $\left.d_{6}\right): \delta=$ 10.98 (br, 1H, NH), 7.80 (d, $J=8.0 \mathrm{~Hz}, 1 \mathrm{H}, \mathrm{ArH}), 7.73$ (d, $J=8.0$ 
Hz, 1H, ArH), 7.64 (s, 1H, ArH), 7.49 (s, 1H, ArH), 7.38 (t, $J=8.0 \mathrm{~Hz}, 1 \mathrm{H}, \operatorname{ArH})$, 7.23-7.31(m, 4H, ArH), $7.08(\mathrm{~d}, J=8.0 \mathrm{~Hz}, 1 \mathrm{H}, \operatorname{ArH}), 6.99-7.03(\mathrm{~m}, 1 \mathrm{H}, \operatorname{ArH})$, 6.36-6.45 (m, 3H, ArH), 5.75 (s, 1H, ArH), 5.49 (br, 1H, OH), 2.35 (AB, $J=17.2 \mathrm{~Hz}$, $\left.1 \mathrm{H}, \mathrm{CH}_{2}\right), 2.12\left(\mathrm{AB}, J=16.4 \mathrm{~Hz}, 1 \mathrm{H}, \mathrm{CH}_{2}\right), 2.04\left(\mathrm{AB}, J=16.0 \mathrm{~Hz}, 1 \mathrm{H}, \mathrm{CH}_{2}\right), 2.35(\mathrm{AB}$, $\left.J=17.2 \mathrm{~Hz}, 1 \mathrm{H}, \mathrm{CH}_{2}\right), 1.88\left(\mathrm{AB}, J=17.2 \mathrm{~Hz}, 1 \mathrm{H}, \mathrm{CH}_{2}\right), 1.15\left(\mathrm{~s}, 3 \mathrm{H}, \mathrm{CH}_{3}\right), 0.89$ (s, 3H, $\left.\mathrm{CH}_{3}\right) ;{ }^{13} \mathrm{C}$ NMR (100 MHz, DMSO- $\left.d_{6}\right): \delta=189.3,162.2,161.7\left(\mathrm{~d},{ }^{1} J_{\mathrm{C}-\mathrm{F}}=243.0 \mathrm{~Hz}\right)$, $158.5\left(\mathrm{~d},{ }^{1} J_{\mathrm{C}-\mathrm{F}}=231.0 \mathrm{~Hz}\right), 147.1,141.7,137.0\left(\mathrm{~d},{ }^{3} J_{\mathrm{C}-\mathrm{F}}=13.0 \mathrm{~Hz}\right), 136.2,133.5$, $133.4,132.3,132.1,131.2,128.9,127.8,125.4,124.8,123.1,122.0,119.9,116.3\left(\mathrm{~d},{ }^{2} J\right.$ $\left.{ }_{\mathrm{C}-\mathrm{F}}=22.0 \mathrm{~Hz}\right), 116.1\left(\mathrm{~d},{ }^{2} J_{\mathrm{C}-\mathrm{F}}=22.0 \mathrm{~Hz}\right), 106.7,106.4,97.5\left(\mathrm{~d},{ }^{2} J_{\mathrm{C}-\mathrm{F}}=25.0 \mathrm{~Hz}\right), 97.3$ $\left(\mathrm{d},{ }^{2} J_{\mathrm{C}-\mathrm{F}}=25.0 \mathrm{~Hz}\right), 63.9,51.5,37.5,33.8,29.9,27.6$; HRMS (ESI-TOF): $\mathrm{m} / z$ calcd for $\mathrm{C}_{34} \mathrm{H}_{27} \mathrm{~F}_{2} \mathrm{~N}_{2} \mathrm{O}_{2}^{+}\left[(\mathrm{M}+\mathrm{H})^{+}\right]$, 533.2035; found, 533.2034.

7-(4-fluorophenyl)-6b-hydroxy-11b-(1H-indol-3-yl)-9,9-dimethyl-8,9,10,11b-tetrahy dro-6bH-acenaphtho[1,2-b]indol-11(7H)-one $(4 \mathrm{~m})$

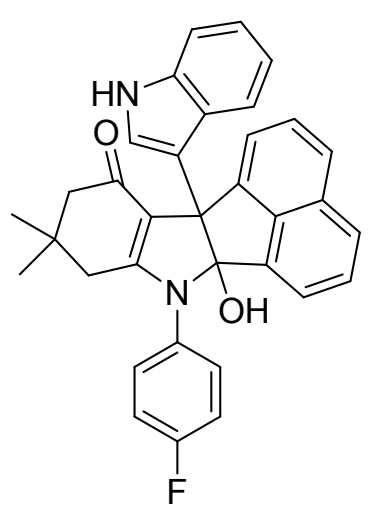

White solid; Mp 231-233 ${ }^{\circ} \mathrm{C}$; IR (KBr): 3428, 2956, 1612, 1567, 1507, 1396, 1220, $780 \mathrm{~cm}^{-1} ;{ }^{1} \mathrm{H}$ NMR (400 MHz, DMSO- $\left.d_{6}\right): \delta=$ $10.92(\mathrm{br}, 1 \mathrm{H}, \mathrm{NH}), 7.80(\mathrm{~d}, J=8.0 \mathrm{~Hz}, 1 \mathrm{H}, \mathrm{ArH}), 7.72(\mathrm{~d}, J=8.0$ $\mathrm{Hz}, 1 \mathrm{H}, \mathrm{ArH}), 7.70-7.73(\mathrm{~m}, 1 \mathrm{H}, \mathrm{ArH}), 7.48-7.66$ (m, 1H, ArH), $7.38(\mathrm{t}, J=8.0 \mathrm{~Hz}, 1 \mathrm{H}, \mathrm{ArH}), 7.16-7.31(\mathrm{~m}, 6 \mathrm{H}, \mathrm{ArH}), 6.86(\mathrm{~s}, 1 \mathrm{H}$, ArH), 6.45 (d, $J=8.0 \mathrm{~Hz}, 1 \mathrm{H}, \mathrm{ArH}), 6.37$ (s, 1H, ArH), 6.35-6.42 (m, 1H, ArH), 5.58 (br, 1H, OH), $2.34\left(\mathrm{AB}, J=16.0 \mathrm{~Hz}, 1 \mathrm{H}, \mathrm{CH}_{2}\right)$, $2.13\left(\mathrm{AB}, J=16.0 \mathrm{~Hz}, 1 \mathrm{H}, \mathrm{CH}_{2}\right), 2.03-2.07\left(\mathrm{~m}, 1 \mathrm{H}, \mathrm{CH}_{2}\right), 1.85-1.90\left(\mathrm{~m}, 1 \mathrm{H}, \mathrm{CH}_{2}\right), 1.16$ (s, $\left.3 \mathrm{H}, \mathrm{CH}_{3}\right), 0.89$ (s, $\left.3 \mathrm{H}, \mathrm{CH}_{3}\right) ;{ }^{13} \mathrm{C}$ NMR $\left(100 \mathrm{MHz}, \mathrm{DMSO}-d_{6}\right): \delta=189.3,161.7\left(\mathrm{~d},{ }^{1} J\right.$ $\left.{ }_{\mathrm{C}-\mathrm{F}}=243.0 \mathrm{~Hz}\right), 161.9,141.8,137.3,136.3,133.5,132.2\left(\mathrm{~d},{ }^{3} J_{\mathrm{C}-\mathrm{F}}=9.0 \mathrm{~Hz}\right), 131.2$, $128.9,128.0,127.7,125.4,123.0,122.1,120.4,119.9,118.1,116.3\left(\mathrm{~d},{ }^{2} J_{\mathrm{C}-\mathrm{F}}=22.0 \mathrm{~Hz}\right)$, $116.1\left(\mathrm{~d},{ }^{2} J_{\mathrm{C}-\mathrm{F}}=22.0 \mathrm{~Hz}\right), 111.5,106.6,64.1,37.5,33.8,30.0,28.4,27.7$; HRMS (ESI-TOF): $m / z$ calcd for $\mathrm{C}_{34} \mathrm{H}_{28} \mathrm{FN}_{2} \mathrm{O}_{2}{ }^{+}\left[(\mathrm{M}+\mathrm{H})^{+}\right], 515.2129$; found, 515.2129.

7-(4-chlorophenyl)-11b-(6-fluoro-1 $H$-indol-3-yl)-6b-hydroxy-9,9-dimethyl-8,9,10,11b -tetrahydro-6bH-acenaphtho[1,2-b]indol-11(7H)-one (4n) 


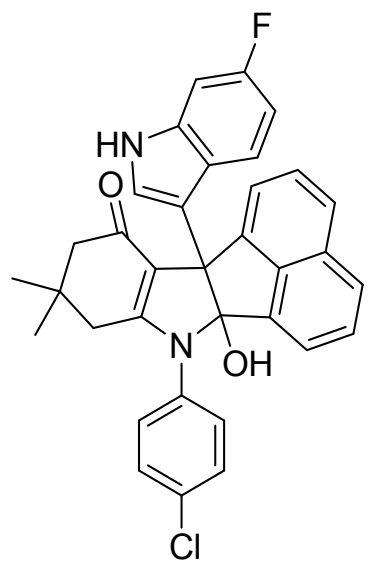

White solid; Mp 227-229 ${ }^{\circ} \mathrm{C}$; IR (KBr): 3440, 2952, 1557, 1419, $1328,1109,1062,785 \mathrm{~cm}^{-1} ;{ }^{1} \mathrm{H}$ NMR (400 MHz, DMSO- $\left.d_{6}\right): \delta$ $=10.98(\mathrm{br}, 1 \mathrm{H}, \mathrm{NH}), 7.80(\mathrm{~d}, J=8.0 \mathrm{~Hz}, 1 \mathrm{H}, \mathrm{ArH}), 7.72(\mathrm{~d}, J=$ $8.0 \mathrm{~Hz}, 1 \mathrm{H}, \mathrm{ArH}), 7.57-7.60(\mathrm{~m}, 1 \mathrm{H}, \mathrm{ArH}), 7.51(\mathrm{~d}, J=8.4 \mathrm{~Hz}$, $3 \mathrm{H}, \mathrm{ArH}), 7.38(\mathrm{t}, J=8.0 \mathrm{~Hz}, 1 \mathrm{H}, \mathrm{ArH}), 7.24(\mathrm{~m}, 2 \mathrm{H}, \mathrm{ArH})$, 7.05-7.09 (m, 2H, ArH), 6.46-6.51 (m, 2H, ArH), 6.30 (s, 1H, ArH), 5.49 (br, 1H, OH), 2.39 (AB, $\left.J=16.8 \mathrm{~Hz}, 1 \mathrm{H}, \mathrm{CH}_{2}\right), 2.12$ $\left(\mathrm{AB}, J=16.8 \mathrm{~Hz}, 1 \mathrm{H}, \mathrm{CH}_{2}\right), 1.98-2.06\left(\mathrm{~m}, 1 \mathrm{H}, \mathrm{CH}_{2}\right), 1.87-1.91$ $\left(\mathrm{m}, 1 \mathrm{H}, \mathrm{CH}_{2}\right), 1.14\left(\mathrm{~s}, 3 \mathrm{H}, \mathrm{CH}_{3}\right), 0.90\left(\mathrm{~s}, 3 \mathrm{H}, \mathrm{CH}_{3}\right) ;{ }^{13} \mathrm{C} \mathrm{NMR}\left(100 \mathrm{MHz}, \mathrm{DMSO}-d_{6}\right): \delta=$ $189.5,161.9,158.6\left(\mathrm{~d},{ }^{1} J_{\mathrm{C}-\mathrm{F}}=230.9 \mathrm{~Hz}\right), 146.9,141.6,137.1\left(\mathrm{~d},{ }^{3} J_{\mathrm{C}-\mathrm{F}}=12.0 \mathrm{~Hz}\right)$, 136.3, 136.2, 132.6, 131.7, 131.2, 129.4, 128.9, 127.8, 125.5, 123.1, 122.1, 119.9, 106.7, $106.5\left(\mathrm{~d},{ }^{2} J_{\mathrm{C}-\mathrm{F}}=23.0 \mathrm{~Hz}\right), 97.4\left(\mathrm{~d},{ }^{2} J_{\mathrm{C}-\mathrm{F}}=25.0 \mathrm{~Hz}\right), 64.0,51.5,37.5,33.9,30.0,27.6$; HRMS (ESI-TOF): $m / z$ calcd for $\mathrm{C}_{34} \mathrm{H}_{27} \mathrm{ClFN}_{2} \mathrm{O}_{2}{ }^{+}\left[(\mathrm{M}+\mathrm{H})^{+}\right]$, 549.1740; found, 547.1793.

7-(4-chlorophenyl)-6b-hydroxy-11b-(1H-indol-3-yl)-9,9-dimethyl-8,9,10,11b-tetrahy dro-6bH-acenaphtho[1,2-b]indol-11(7H)-one (4o)

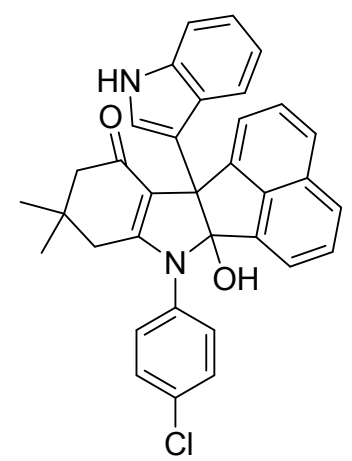

White solid; Mp 216-218 ${ }^{\circ} \mathrm{C}$; IR (KBr): 3454, 2957, 1600, 1557, 1495, 1093, 1018, $793 \mathrm{~cm}^{-1} ;{ }^{1} \mathrm{H}$ NMR (400 MHz, DMSO- $\left.d_{6}\right): \delta=$ $10.90(\mathrm{br}, 1 \mathrm{H}, \mathrm{NH}), 7.80(\mathrm{~d}, J=8.0 \mathrm{~Hz}, 1 \mathrm{H}, \mathrm{ArH}), 7.73(\mathrm{~d}, J=8.0$ $\mathrm{Hz}, 1 \mathrm{H}, \mathrm{ArH}), 7.47-7.59(\mathrm{~m}, 4 \mathrm{H}, \mathrm{ArH}), 7.38(\mathrm{t}, J=7.2 \mathrm{~Hz}, 1 \mathrm{H}$, ArH), 7.20-7.30 (m, 3H, ArH), 6.85-7.04 (m, 2H, ArH), 6.47 (d, $J=6.8 \mathrm{~Hz}, 1 \mathrm{H}, \mathrm{ArH}), 6.41$ (s, 1H, ArH), 5.56 (br, 1H, OH), 2.39 $\left(\mathrm{AB}, J=17.2 \mathrm{~Hz}, 1 \mathrm{H}, \mathrm{CH}_{2}\right), 2.13\left(\mathrm{AB}, J=16.0 \mathrm{~Hz}, 1 \mathrm{H}, \mathrm{CH}_{2}\right)$, $2.04\left(\mathrm{AB}, J=17.2 \mathrm{~Hz}, 1 \mathrm{H}, \mathrm{CH}_{2}\right), 1.89\left(\mathrm{AB}, J=16.8 \mathrm{~Hz}, 1 \mathrm{H}, \mathrm{CH}_{2}\right), 1.16\left(\mathrm{~s}, 3 \mathrm{H}, \mathrm{CH}_{3}\right)$, 0.90 (s, $\left.3 \mathrm{H}, \mathrm{CH}_{3}\right) ;{ }^{13} \mathrm{C}$ NMR (100 MHz, DMSO- $\left.d_{6}\right): \delta=189.4,161.4,147.0,141.7,137.2$, 136.4, 136.2, 132.6, 131.7, 131.2, 129.4, 128.9, 127.7, 125.4, 123.0, 122.1, 120.4, 119.8, 118.1, 106.7, 64.1, 51.6, 37.6, 33.9, 30.0, 27.6; HRMS (ESI-TOF): $\mathrm{m} / \mathrm{z}$ calcd for $\mathrm{C}_{34} \mathrm{H}_{28} \mathrm{ClN}_{2} \mathrm{O}_{2}^{+}\left[(\mathrm{M}+\mathrm{H})^{+}\right]$, 531.1834; found, 531.1835.

7-(3-chlorophenyl)-6b-hydroxy-11b-(1H-indol-3-yl)-9,9-dimethyl-8,9,10,11b-tetrahy 
dro-6bH-acenaphtho[1,2-b]indol-11(7H)-one $(4 p)$

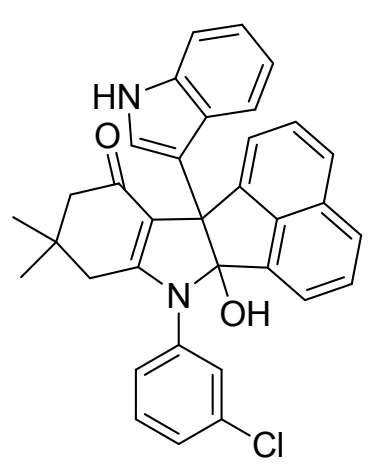

White solid; $\mathrm{Mp} 230-232{ }^{\circ} \mathrm{C}$; IR (KBr): 3438, 2961, 1624, 1479, $1397,1326,1253,778 \mathrm{~cm}^{-1} ;{ }^{1} \mathrm{H}$ NMR (400 MHz, DMSO- $\left.d_{6}\right): \delta=$ 10.92 (br, 1H, NH), 7.81 (d, $J=8.0 \mathrm{~Hz}, 1 \mathrm{H}, \mathrm{ArH}), 7.73$ (d, $J=8.0$ $\mathrm{Hz}, 1 \mathrm{H}, \mathrm{ArH}), 7.35-7.59$ (m, 5H, ArH), 7.30 (d, $J=8.0 \mathrm{~Hz}, 1 \mathrm{H}$, ArH), 7.04-7.09 (m, 2H, ArH), 6.86 (s, 1H, ArH), 6.42-6.46 (m, 2H, ArH), $5.72(\mathrm{br}, 1 \mathrm{H}, \mathrm{OH}), 2.44\left(\mathrm{~s}, 1 \mathrm{H}, \mathrm{CH}_{2}\right), 1.87-2.16(\mathrm{~m}, 3 \mathrm{H}$, $\left.\mathrm{CH}_{2}\right), 1.17\left(\mathrm{~s}, 3 \mathrm{H}, \mathrm{CH}_{3}\right), 0.92\left(\mathrm{~s}, 3 \mathrm{H}, \mathrm{CH}_{3}\right) ;{ }^{13} \mathrm{C} \mathrm{NMR}(100 \mathrm{MHz}$, DMSO- $\left.d_{6}\right): \delta=189.6,162.4,141.6,138.9,137.2,136.2,131.2,130.8,129.8,129.0$, $128.6,128.0,127.7,125.5,123.1,122.1,120.4,119.7,118.1,111.6,106.8,64.2,51.5$, 39.3, 37.5, 33.9, 30.1; HRMS (ESI-TOF): $m / z$ calcd for $\mathrm{C}_{34} \mathrm{H}_{28} \mathrm{ClN}_{2} \mathrm{O}_{2}{ }^{+}\left[(\mathrm{M}+\mathrm{H})^{+}\right]$, 531.1834; found, 531.1834.

7-(4-bromophenyl)-6b-hydroxy-11b-(1H-indol-3-yl)-9,9-dimethyl-8,9,10,11b-tetrahy dro-6bH-acenaphtho[1,2-b]indol-11(7H)-one (4q)

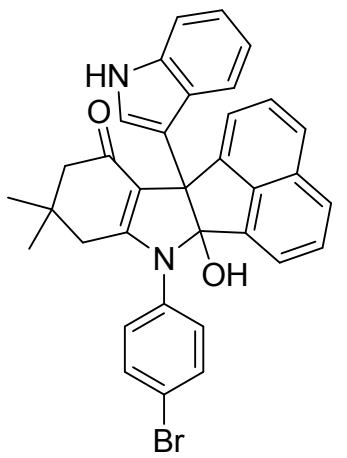

White solid; $\mathrm{Mp} 232-233{ }^{\circ} \mathrm{C}$; IR (KBr): 3429, 2955, 1616, 1559, $1400,1249,1183,793 \mathrm{~cm}^{-1} ;{ }^{1} \mathrm{H}$ NMR (400 MHz, DMSO-d $): \delta=$ 10.92 (br, 1H, NH), 7.80 (d, $J=8.4 \mathrm{~Hz}, 1 \mathrm{H}, \operatorname{ArH}), 7.72$ (d, $J=8.0$ $\mathrm{Hz}, 1 \mathrm{H}, \mathrm{ArH}), 7.64(\mathrm{~d}, J=8.4 \mathrm{~Hz}, 2 \mathrm{H}, \mathrm{ArH}), 7.62-7.70(\mathrm{~m}, 1 \mathrm{H}$, ArH), 7.47-7.51 (m, 1H, ArH), 7.38 (t, $J=7.6 \mathrm{~Hz}, 1 \mathrm{H}, \mathrm{ArH}), 7.29$ $(\mathrm{d}, J=8.4 \mathrm{~Hz}, 1 \mathrm{H}, \mathrm{ArH}), 7.15-7.21(\mathrm{~m}, 2 \mathrm{H}, \mathrm{ArH}), 6.83-7.14(\mathrm{~m}$, 2H, ArH), 6.49 (d, J=7.2 Hz, 1H, ArH), 6.27-6.44 (m, 2H, ArH), 5.55 (br, 1H, OH), $2.39\left(\mathrm{AB}, J=16.8 \mathrm{~Hz}, 1 \mathrm{H}, \mathrm{CH}_{2}\right), 2.14\left(\mathrm{AB}, J=16.0 \mathrm{~Hz}, 1 \mathrm{H}, \mathrm{CH}_{2}\right), 2.02-2.07(\mathrm{~m}, 1 \mathrm{H}$, $\left.\mathrm{CH}_{2}\right), 1.88-1.92\left(\mathrm{~m}, 1 \mathrm{H}, \mathrm{CH}_{2}\right), 1.16\left(\mathrm{~s}, 3 \mathrm{H}, \mathrm{CH}_{3}\right), 0.90\left(\mathrm{~s}, 3 \mathrm{H}, \mathrm{CH}_{3}\right) ;{ }^{13} \mathrm{C} \mathrm{NMR}(100 \mathrm{MHz}$, DMSO- $\left.d_{6}\right): \delta=189.4,161.9,141.7,137.2,136.8,136.2,132.4,132.0,131.2,128.9$, $127.9,127.8,125.4,123.0,122.1,121.1,120.4,119.8,118.1,111.5,106.7,64.2,51.5$, 37.6, 33.9, 30.0, 27.6; HRMS (ESI-TOF): $m / z$ calcd for $\mathrm{C}_{34} \mathrm{H}_{28} \mathrm{BrN}_{2} \mathrm{O}_{2}{ }^{+}\left[(\mathrm{M}+\mathrm{H})^{+}\right]$, 575.1329; found, 575.1327.

11b-(6-fluoro-1H-indol-3-yl)-6b-hydroxy-9,9-dimethyl-7-phenyl-8,9,10,11b-tetrahyd 
ro-6bH-acenaphtho[1,2-b]indol-11(7H)-one $(4 r)$

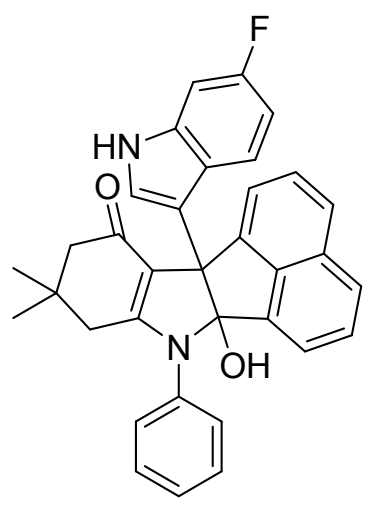

White solid; Mp 239-240 우 ; IR (KBr): 3443, 2962, 1564, 1497, $1402,1245,1144,793 \mathrm{~cm}^{-1}$; ${ }^{1} \mathrm{H}$ NMR (400 MHz, DMSO- $\left.d_{6}\right): \delta=$ 10.97 (br, 1H, NH), 7.79 (d, $J=8.0 \mathrm{~Hz}, 1 \mathrm{H}, \mathrm{ArH}), 7.72$ (d, $J=8.0$ $\mathrm{Hz}, 1 \mathrm{H}, \mathrm{ArH}), 7.62-7.70$ (m, 2H, ArH), 7.42-7.67 (m, 4H, ArH), $7.36(\mathrm{t}, J=8.0 \mathrm{~Hz}, 1 \mathrm{H}, \mathrm{ArH}), 7.16-7.22(\mathrm{~m}, 2 \mathrm{H}, \mathrm{ArH}), 7.07$ (d, $J$ $=8.0 \mathrm{~Hz}, 1 \mathrm{H}, \mathrm{ArH}), 7.06-7.09(\mathrm{~m}, 1 \mathrm{H}, \mathrm{ArH}), 6.47(\mathrm{~s}, 1 \mathrm{H}, \mathrm{ArH})$, $6.40(\mathrm{~d}, J=8.0 \mathrm{~Hz}, 1 \mathrm{H}, \mathrm{ArH}), 6.30-6.34$ (m, 1H, ArH), 5.51 (br, $1 \mathrm{H}, \mathrm{OH}), 2.37\left(\mathrm{AB}, J=16.0 \mathrm{~Hz}, 1 \mathrm{H}, \mathrm{CH}_{2}\right), 2.12\left(\mathrm{AB}, J=16.0 \mathrm{~Hz}, 1 \mathrm{H}, \mathrm{CH}_{2}\right), 2.04(\mathrm{AB}$, $\left.J=16.0 \mathrm{~Hz}, 1 \mathrm{H}, \mathrm{CH}_{2}\right), 2.00\left(\mathrm{AB}, J=16.0 \mathrm{~Hz}, 1 \mathrm{H}, \mathrm{CH}_{2}\right), 1.14\left(\mathrm{~s}, 3 \mathrm{H}, \mathrm{CH}_{3}\right), 0.89$ (s, 3H, $\left.\mathrm{CH}_{3}\right) ;{ }^{13} \mathrm{C}$ NMR (100 MHz, DMSO- $\left.d_{6}\right): \delta=189.2,162.3,158.4\left(\mathrm{~d},{ }^{1} J_{\mathrm{C}-\mathrm{F}}=184.0 \mathrm{~Hz}\right)$, $141.8,137.3,137.0\left(\mathrm{~d},{ }^{3} J_{\mathrm{C}-\mathrm{F}}=9.0 \mathrm{~Hz}\right), 136.2,131.2,130.0,129.3,128.9,128.1,127.7$, 125.3, 123.0, 122.0, 120.4, 119.9, 106.7, $106.5\left(\mathrm{~d},{ }^{2} J_{\mathrm{C}-\mathrm{F}}=24.0 \mathrm{~Hz}\right), 97.3\left(\mathrm{~d},{ }^{2} J_{\mathrm{C}-\mathrm{F}}=\right.$ $25.0 \mathrm{~Hz}$ ), 64.0, 51.5, 37.7, 33.8, 29.9, 27.7; HRMS (ESI-TOF): $\mathrm{m} / \mathrm{z}$ calcd for $\mathrm{C}_{34} \mathrm{H}_{28} \mathrm{FN}_{2} \mathrm{O}_{3}{ }^{+}\left[(\mathrm{M}+\mathrm{H})^{+}\right], 515.2129$; found, 515.2129.

6b-hydroxy-11b-(1H-indol-3-yl)-9,9-dimethyl-7-phenyl-8,9,10,11b-tetrahydro-6bH-a cenaphtho[1,2-b]indol-11(7H)-one $(4 \mathrm{~s})$

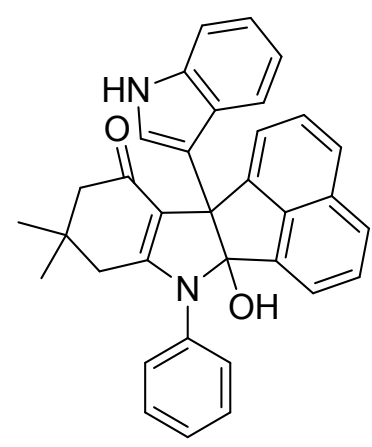

White solid; Mp 219-221 ${ }^{\circ} \mathrm{C}$; IR (KBr): 3457, 2959, 1618, 1561, 1400, 1251, 1185, $793 \mathrm{~cm}^{-1} ;{ }^{1} \mathrm{H}$ NMR (400 MHz, DMSO- $\left.d_{6}\right): \delta=$ 10.89 (br, 1H, NH), 7.79 (d, $J=8.0 \mathrm{~Hz}, 1 \mathrm{H}, \operatorname{ArH}), 7.72$ (d, $J=$ $8.0 \mathrm{~Hz}, 1 \mathrm{H}, \mathrm{ArH}), 7.40-7.59$ (m, 5H, ArH), 7.34 (t, $J=7.2 \mathrm{~Hz}$, 1H, ArH), 7.29 (d, $J=8.0 \mathrm{~Hz}, 1 \mathrm{H}, \operatorname{ArH}), 7.19$ (s, 2H, ArH), 6.82-6.90 (m, 2H, ArH), 6.38-6.44 (m, 1H, ArH), 6.39 (d, $J=$ $7.2 \mathrm{~Hz}, 1 \mathrm{H}, \mathrm{ArH}), 6.33$ (s, 1H, ArH), 5.56 (br, $1 \mathrm{H}, \mathrm{OH}), 2.38$ (AB, $J=16.8 \mathrm{~Hz}, 1 \mathrm{H}, \mathrm{CH}_{2}$ ), $2.13\left(\mathrm{AB}, J=16.0 \mathrm{~Hz}, 1 \mathrm{H}, \mathrm{CH}_{2}\right), 2.03\left(\mathrm{AB}, J=16.0 \mathrm{~Hz}, 1 \mathrm{H}, \mathrm{CH}_{2}\right), 1.88(\mathrm{AB}, J=17.2$ $\left.\mathrm{Hz}, 1 \mathrm{H}, \mathrm{CH}_{2}\right), 1.15\left(\mathrm{~s}, 3 \mathrm{H}, \mathrm{CH}_{3}\right), 0.89$ (s, $\left.3 \mathrm{H}, \mathrm{CH}_{3}\right) ;{ }^{13} \mathrm{C}$ NMR (100 MHz, DMSO- $\left.d_{6}\right): \delta$ $=189.2,162.0,147.3,141.9,137.4,137.2,136.3,131.2,130.0,129.3,128.9,128.1,127.6$, $125.3,122.9,122.0,120.4,119.9,118.0,111.5,106.7,64.1,51.6,37.7,33.9,30.0,27.7$; 
HRMS (ESI-TOF): $m / z$ calcd for $\mathrm{C}_{32} \mathrm{H}_{29} \mathrm{~N}_{2} \mathrm{O}_{2}{ }^{+}\left[(\mathrm{M}+\mathrm{H})^{+}\right]$, 497.2224; found, 497.2224.

11b-(6-fluoro-1H-indol-3-yl)-6b-hydroxy-9,9-dimethyl-7-(p-tolyl)-8,9,10,11b-tetrahy dro-6bH-acenaphtho[1,2-b]indol-11(7H)-one $(4 t)$

White solid; Mp 228-231 ${ }^{\circ} \mathrm{C}$; IR (KBr): 3428, 2957, 1631, 1598, 1511, 1245, 1142, 784 $\mathrm{cm}^{-1}$; ${ }^{1} \mathrm{H}$ NMR (400 MHz, DMSO- $d_{6}$ ): $\delta=10.97$ (br, 1H, NH), 7.78 (d, $J=8.0 \mathrm{~Hz}, 1 \mathrm{H}$, ArH), $7.72(\mathrm{~d}, J=8.4 \mathrm{~Hz}, 1 \mathrm{H}, \mathrm{ArH}), 7.62(\mathrm{~s}, 1 \mathrm{H}, \mathrm{ArH}), 7.49(\mathrm{~s}, 1 \mathrm{H}, \mathrm{ArH}), 7.36(\mathrm{t}, J=7.6$ Hz, 1H, ArH), 7.23 (d, J=8.0 Hz, 2H, ArH), 7.04-7.10(m, 4H, ArH), 6.47 (d, J=6.8 Hz, 1H, ArH), 6.37 (s, 1H, ArH), 6.32-6.38 (m, 1H, ArH), 5.56 (br, 1H, OH), 2.35 (s, 3H,

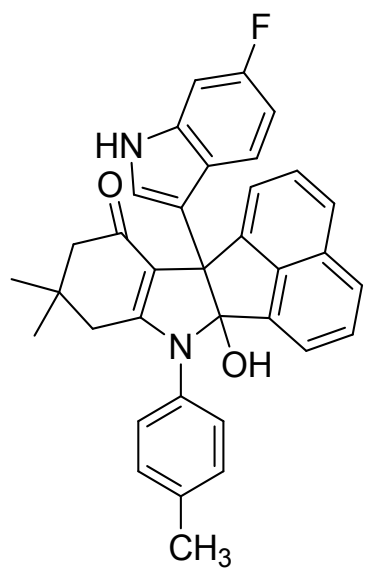

$\left.\mathrm{CH}_{3}\right), 2.31\left(\mathrm{AB}, J=17.6 \mathrm{~Hz}, 1 \mathrm{H}, \mathrm{CH}_{2}\right), 1.91-2.12\left(\mathrm{~m}, 2 \mathrm{H}, \mathrm{CH}_{2}\right)$, 1.85-1.90 (m, 1H, $\left.\mathrm{CH}_{2}\right), 1.13\left(\mathrm{~s}, 3 \mathrm{H}, \mathrm{CH}_{3}\right), 0.87\left(\mathrm{~s}, 3 \mathrm{H}, \mathrm{CH}_{3}\right) ;{ }^{13} \mathrm{C}$ NMR $\left(100 \mathrm{MHz}, \mathrm{DMSO}-d_{6}\right): \delta=189.5,162.4,158.6\left(\mathrm{~d},{ }^{1} J_{\mathrm{C}-\mathrm{F}}=\right.$ $232.0 \mathrm{~Hz}), 147.2,141.9,137.5,137.0\left(\mathrm{~d},{ }^{3} J_{\mathrm{C}-\mathrm{F}}=13.0 \mathrm{~Hz}\right), 136.2$, $134.5,131.2,129.9,129.9,128.9,127.7,125.3,124.9,123.0$, 122.0, 121.6, 111.5, $106.5\left(\mathrm{~d},{ }^{2} J_{\mathrm{C}-\mathrm{F}}=25.0 \mathrm{~Hz}\right), 97.3\left(\mathrm{~d},{ }^{2} J_{\mathrm{C}-\mathrm{F}}=\right.$ 23.2 Hz), 63.9, 51.5, 37.7, 33.8, 29.9, 27.7, 21.2; HRMS

(ESI-TOF): $m / z$ calcd for $\mathrm{C}_{35} \mathrm{H}_{30} \mathrm{FN}_{2} \mathrm{O}_{2}{ }^{+}\left[(\mathrm{M}+\mathrm{H})^{+}\right]$, 529.2286;

found, 529.2290.

1b-(6-chloro-1H-indol-3-yl)-6b-hydroxy-9,9-dimethyl-7-(p-tolyl)-8,9,10,11b-tetrahyd ro-6bH-acenaphtho[1,2-b]indol-11(7H)-one $(4 \mathrm{u})$

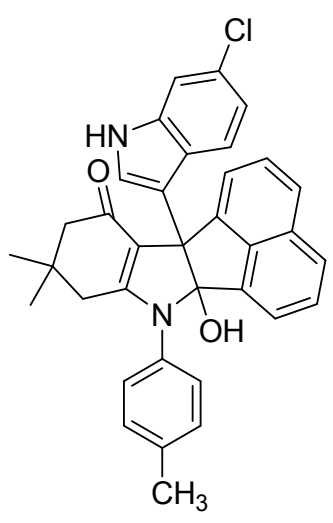

White solid; Mp 265-267 ${ }^{\circ} \mathrm{C}$; IR (KBr): 3367, 2960, 1620, 1514, 1400, 1183, 1065, $784 \mathrm{~cm}^{-1} ;{ }^{1} \mathrm{H}$ NMR (400 MHz, DMSO- $\left.d_{6}\right): \delta=$ 11.03 (br, 1H, NH), 7.79 (d, $J=8.0 \mathrm{~Hz}, 1 \mathrm{H}, \operatorname{ArH}), 7.72$ (d, $J=8.4$ $\mathrm{Hz}, 1 \mathrm{H}, \mathrm{ArH}), 7.41-7.65$ (m, 2H, ArH), 7.33-7.38 (m, 2H, ArH), $7.23(\mathrm{~d}, J=8.0 \mathrm{~Hz}, 2 \mathrm{H}, \mathrm{ArH}), 7.05-7.09$ (m, 3H, ArH), 6.46 (d, $J=$ $6.8 \mathrm{~Hz}, 1 \mathrm{H}, \mathrm{ArH}), 6.41$ (s, 1H, ArH), 6.40-6.47 (m, 1H, ArH), 5.53 (br, 1H, OH), $2.36\left(\mathrm{~s}, 3 \mathrm{H}, \mathrm{CH}_{3}\right), 2.31\left(\mathrm{AB}, J=16.8 \mathrm{~Hz}, 1 \mathrm{H}, \mathrm{CH}_{2}\right)$, $2.10\left(\mathrm{AB}, J=16.4 \mathrm{~Hz}, 1 \mathrm{H}, \mathrm{CH}_{2}\right), 1.98-2.04\left(\mathrm{~m}, 1 \mathrm{H}, \mathrm{CH}_{2}\right), 1.88(\mathrm{AB}, J=16.0 \mathrm{~Hz}, 1 \mathrm{H}$, $\mathrm{CH}_{2}$ ), 1.13 (s, 3H, $\left.\mathrm{CH}_{3}\right), 0.87\left(\mathrm{~s}, 3 \mathrm{H}, \mathrm{CH}_{3}\right) ;{ }^{13} \mathrm{C} \mathrm{NMR}\left(100 \mathrm{MHz}, \mathrm{DMSO}-d_{6}\right): \delta=189.0$, 
$162.5,147.0,141.8,137.6,137.5,136.2,134.5,131.2,129.9,129.8,128.9,127.7,126.8$, 125.3, 123.1, 121.9, 120.1, 118.4, 111.0, 106.7, 63.8, 51.5, 37.7, 29.9, 27.8, 21.2; HRMS (ESI-TOF): $\mathrm{m} / \mathrm{z}$ calcd for $\mathrm{C}_{35} \mathrm{H}_{30} \mathrm{ClN}_{2} \mathrm{O}_{2}^{+}\left[(\mathrm{M}+\mathrm{H})^{+}\right], 545.1910$; found, 545.1990 .

6b-hydroxy-11b-(1H-indol-3-yl)-9,9-dimethyl-7-(p-tolyl)-8,9,10,11b-tetrahydro-6bHacenaphtho[1,2-b]indol-11(7H)-one $(4 \mathrm{v})$

White solid; Mp 239-240 ${ }^{\circ} \mathrm{C}$; IR (KBr): 3459, 2943, 1606, 1559, 1397, 1326, 1022, 781 $\mathrm{cm}^{-1} ;{ }^{1} \mathrm{H}$ NMR (400 MHz, DMSO- $d_{6}$ ): $\delta=10.89$ (br, $\left.1 \mathrm{H}, \mathrm{NH}\right), 7.78$ (d, $J=8.0 \mathrm{~Hz}, 1 \mathrm{H}$, ArH), $7.71(\mathrm{~d}, J=8.0 \mathrm{~Hz}, 1 \mathrm{H}, \operatorname{ArH}), 7.47-7.62(\mathrm{~m}, 2 \mathrm{H}, \operatorname{ArH}), 7.36(\mathrm{t}, J=8.0 \mathrm{~Hz}, 1 \mathrm{H}$,

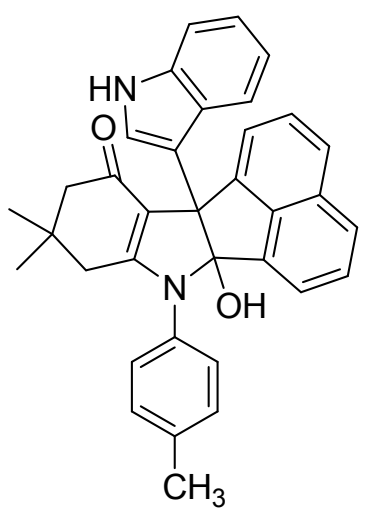
ArH), 7.29 (d, $J=8.0 \mathrm{~Hz}, 1 \mathrm{H}, \operatorname{ArH}), 7.23(\mathrm{~d}, J=8.0 \mathrm{~Hz}, 2 \mathrm{H}$, ArH), 6.85-7.02 (m, 5H, ArH), $6.46(\mathrm{~d}, J=8.0 \mathrm{~Hz}, 1 \mathrm{H}, \mathrm{ArH})$, $6.28(\mathrm{~s}, 1 \mathrm{H}, \mathrm{ArH}), 5.56(\mathrm{br}, 1 \mathrm{H}, \mathrm{OH}), 2.33\left(\mathrm{~s}, 3 \mathrm{H}, \mathrm{CH}_{3}\right)$, 2.29-2.33 (m, 1H, $\left.\mathrm{CH}_{2}\right), 1.85-2.13\left(\mathrm{~m}, 3 \mathrm{H}, \mathrm{CH}_{2}\right), 1.14(\mathrm{~s}, 3 \mathrm{H}$, $\left.\mathrm{CH}_{3}\right), 0.87\left(\mathrm{~s}, 3 \mathrm{H}, \mathrm{CH}_{3}\right) ;{ }^{13} \mathrm{C}$ NMR (100 MHz, DMSO- $\left.d_{6}\right): \delta=$ $189.0,162.3,142.0,137.5,137.3,136.3,134.6,131.2,130.0$, $129.8,128.9,128.0,127.7,125.2,122.9,122.0,120.4,119.9$,

118.0, 111.5, 106.6, 64.1, 51.6, 37.7, 33.8, 30.0, 27.7, 21.2; HRMS (ESI-TOF): $m / z$ calcd for $\mathrm{C}_{35} \mathrm{H}_{31} \mathrm{~N}_{2} \mathrm{O}_{2}^{+}\left[(\mathrm{M}+\mathrm{H})^{+}\right], 511.2380$; found, 511.2416 .

6b-hydroxy-11b-(1H-indol-3-yl)-7-(4-methoxyphenyl)-9,9-dimethyl-8,9,10,11b-tetra hydro-6bH-acenaphtho[1,2-b]indol-11(7H)-one $(4 \mathrm{w})$

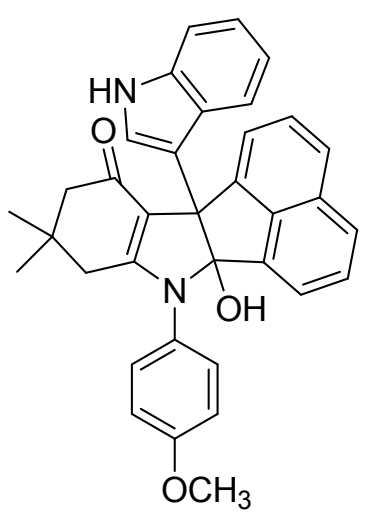

White solid; Mp 210-212 ${ }^{\circ} \mathrm{C}$; IR (KBr): 3426, 2957, 1610, 1557, 1513, 1402, 1249, $784 \mathrm{~cm}^{-1} ;{ }^{1} \mathrm{H}$ NMR (400 MHz, DMSO- $\left.d_{6}\right): \delta=$ 10.89 (br, 1H, NH), 7.79 (d, $J=8.0 \mathrm{~Hz}, 1 \mathrm{H}, \mathrm{ArH}), 7.71$ (d, $J=8.0$ $\mathrm{Hz}, 1 \mathrm{H}, \mathrm{ArH}), 7.58-7.65(\mathrm{~m}, 2 \mathrm{H}, \mathrm{ArH}), 7.38(\mathrm{t}, J=8.0 \mathrm{~Hz}, 1 \mathrm{H}$, ArH), 7.29 (d, $J=8.0 \mathrm{~Hz}, 1 \mathrm{H}, \operatorname{ArH}), 7.23(\mathrm{~d}, J=8.0 \mathrm{~Hz}, 2 \mathrm{H}$, ArH), 6.98-7.12 (m, 3H, ArH), 6.83-6.89 (m, 2H, ArH), 6.47 (d, $J=8.0 \mathrm{~Hz}, 1 \mathrm{H}, \mathrm{ArH}), 6.24$ (s, 1H, ArH), 6.24-6.28 (m, 1H, ArH), $5.54(\mathrm{br}, 1 \mathrm{H}, \mathrm{OH}), 3.79\left(\mathrm{~s}, 3 \mathrm{H}, \mathrm{OCH}_{3}\right), 2.28\left(\mathrm{AB}, J=16.0 \mathrm{~Hz}, 1 \mathrm{H}, \mathrm{CH}_{2}\right), 2.03(\mathrm{AB}, J=$ $\left.16.0 \mathrm{~Hz}, 1 \mathrm{H}, \mathrm{CH}_{2}\right), 1.85-2.03\left(\mathrm{~m}, 2 \mathrm{H}, \mathrm{CH}_{2}\right), 1.15\left(\mathrm{~s}, 3 \mathrm{H}, \mathrm{CH}_{3}\right), 0.87\left(\mathrm{~s}, 3 \mathrm{H}, \mathrm{CH}_{3}\right) ;{ }^{13} \mathrm{C}$ 
NMR (100 MHz, DMSO- $\left.d_{6}\right): \delta=188.9,162.3,142.0,137.5,137.3,136.3,134.6,131.2$, $130.0,129.8,128.9,128.0,127.7,125.2,122.9,122.0,120.4,119.9,118.0,111.5,106.6$, 64.1, 51.6, 37.7, 33.8, 30.0, 27.7, 21.2; HRMS (ESI-TOF): $\mathrm{m} / z$ calcd for $\mathrm{C}_{35} \mathrm{H}_{31} \mathrm{~N}_{2} \mathrm{O}_{3}{ }^{+}$ $\left[(\mathrm{M}+\mathrm{H})^{+}\right], 527.2329$; found, 527.2329 .

\section{6b-hydroxy-9,9-dimethyl-11b-(5-methyl-1 $H$-indol-3-yl)-7-phenyl-8,9,10,11b-tetrahy dro-6bH-acenaphtho[1,2-b]indol-11(7H)-one $(4 x)$}

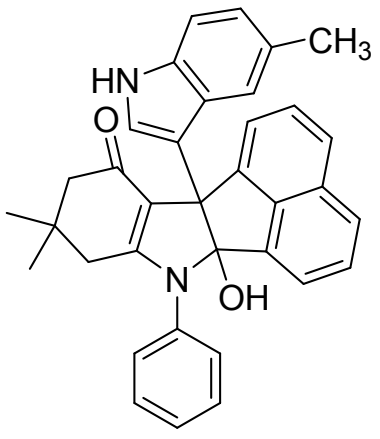

White solid; Mp 231-232 ${ }^{\circ} \mathrm{C}$; IR (KBr): 3453, 2953, 1618, 1561 , 1493, 1404, 1134, 789 $\mathrm{cm}^{-1} ;{ }^{1} \mathrm{H}$ NMR (400 MHz, DMSO- $\left.d_{6}\right): \delta=$ 10.74 (br, 1H, NH), 7.79 (d, $J=8.0 \mathrm{~Hz}, 1 \mathrm{H}, \mathrm{ArH}), 7.73$ (d, $J=$ $8.0 \mathrm{~Hz}, 1 \mathrm{H}, \mathrm{ArH}), 7.38-7.51(\mathrm{~m}, 5 \mathrm{H}, \mathrm{ArH}), 7.34$ (t, $J=8.0 \mathrm{~Hz}$, 1H, ArH), 7.17-7.23 (m, 3H, ArH), 6.60-6.68 (m, 2H, ArH), 6.41 (d, $J=8.0 \mathrm{~Hz}, 1 \mathrm{H}, \operatorname{ArH}), 6.29$ (s, 1H, ArH), 5.31 (br, 1H, $\mathrm{OH}), 2.38\left(\mathrm{AB}, J=16.0 \mathrm{~Hz}, 1 \mathrm{H}, \mathrm{CH}_{2}\right), 2.12\left(\mathrm{AB}, J=16.0 \mathrm{~Hz}, 1 \mathrm{H}, \mathrm{CH}_{2}\right), 1.77-2.09(\mathrm{~m}$, $\left.5 \mathrm{H}, \mathrm{CH}_{2}+\mathrm{CH}_{3}\right), 1.16\left(\mathrm{~s}, 3 \mathrm{H}, \mathrm{CH}_{3}\right), 0.89\left(\mathrm{~s}, 3 \mathrm{H}, \mathrm{CH}_{3}\right) ;{ }^{13} \mathrm{C}$ NMR $\left(100 \mathrm{MHz}, \mathrm{DMSO}-d_{6}\right): \delta$ $=188.6,161.4,141.4,136.9,135.8,135.2,130.7,129.6,128.8,128.3,127.5,127.1$, $125.4,124.7,122.4,121.6,119.4,110.7,106.1,63.7,51.1,38.9,37.3,33.3,29.6,21.3$; HRMS (ESI-TOF): $m / z$ calcd for $\mathrm{C}_{35} \mathrm{H}_{31} \mathrm{~N}_{2} \mathrm{O}_{2}{ }^{+}\left[(\mathrm{M}+\mathrm{H})^{+}\right], 511.2380$; found, 511.2380. 


\section{Copies of Original ${ }^{1} \mathrm{H}$ and ${ }^{13} \mathrm{C}$ NMR Spectra}

$\underline{{ }^{1} \mathrm{H} N M R \text { and }{ }^{13} \mathrm{C} \text { NMR spectra for compound } 4}$

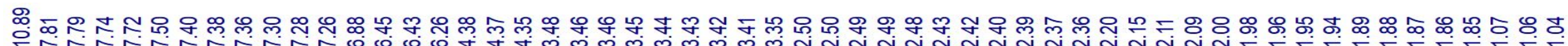

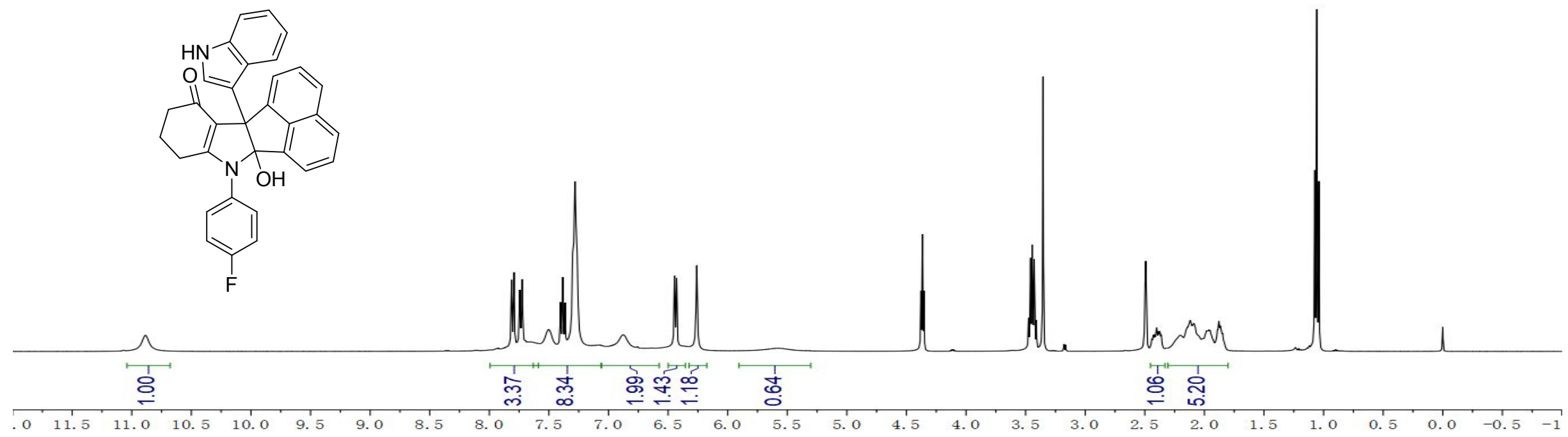

Figure S1. ${ }^{1} \mathrm{H}$ NMR $\left(400 \mathrm{MHz}, \mathrm{DMSO}-d_{6}\right)$ spectra of compound $4 \mathrm{a}$ 


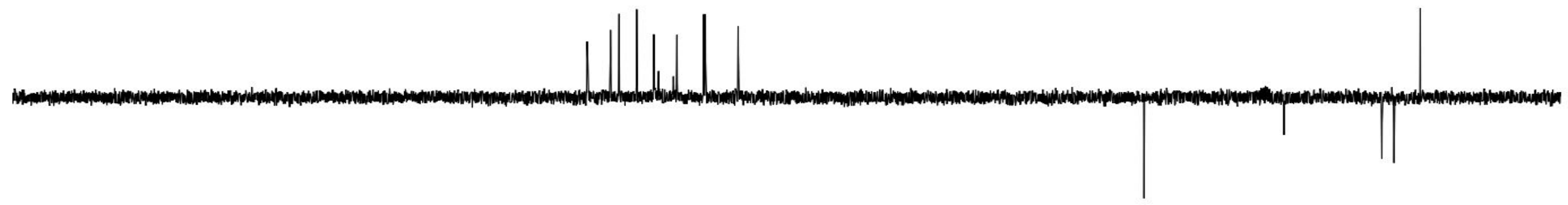

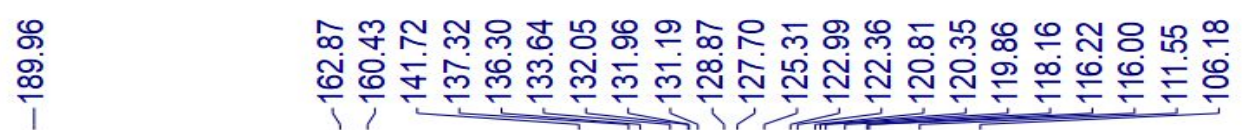

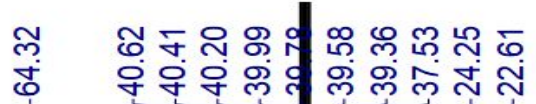

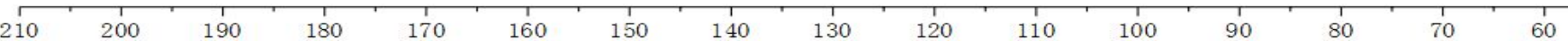

Figure S2. ${ }^{13} \mathrm{C}$ NMR $\left(400 \mathrm{MHz}, \mathrm{DMSO}-d_{6}\right)$ spectra of compound $4 a$ 


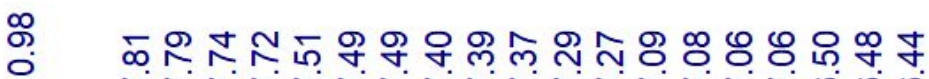

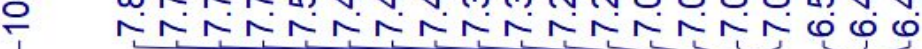

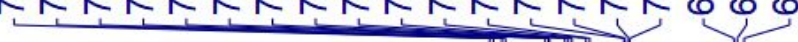
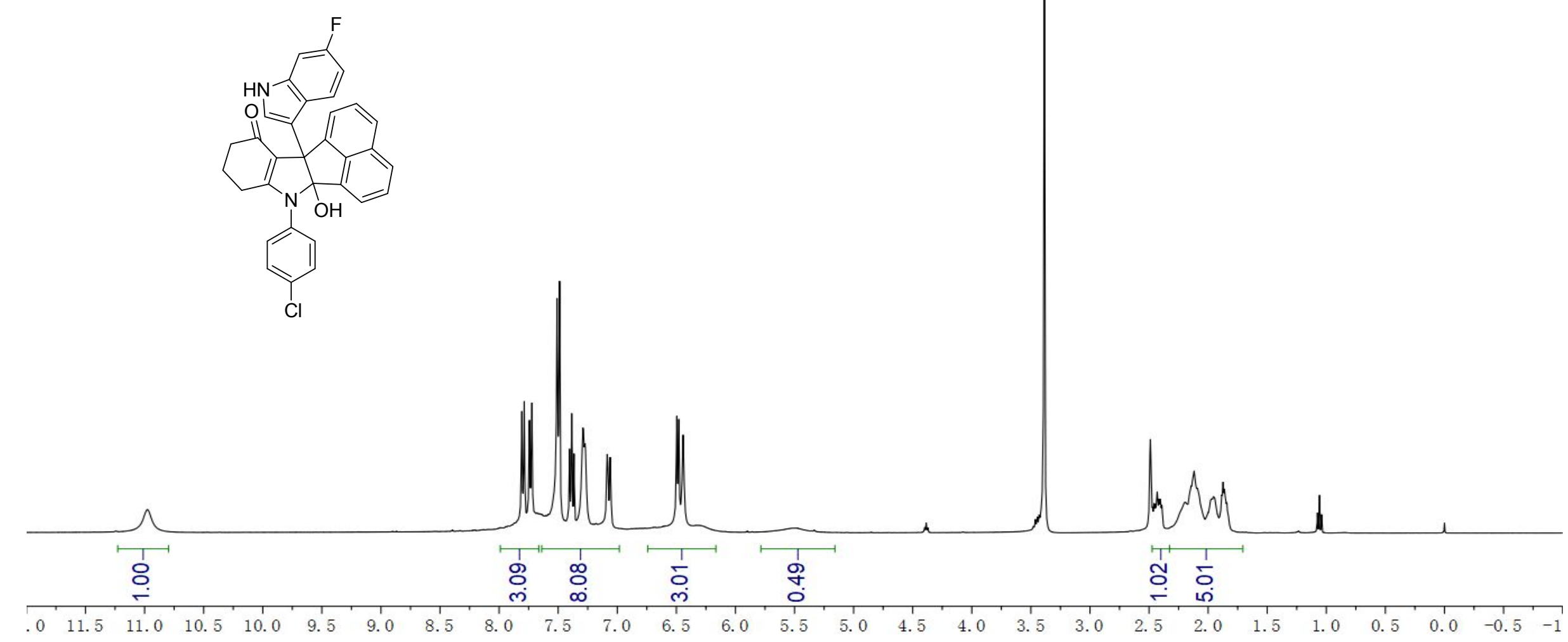

Figure S3. ${ }^{1} \mathrm{H}$ NMR $\left(400 \mathrm{MHz}, \mathrm{DMSO}-d_{6}\right)$ spectra of compound $\mathbf{4 b}$ 


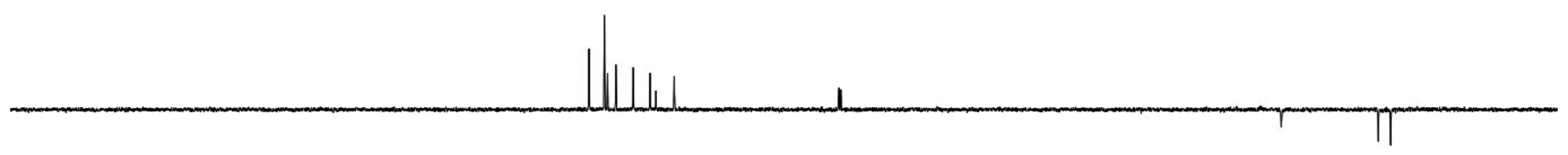

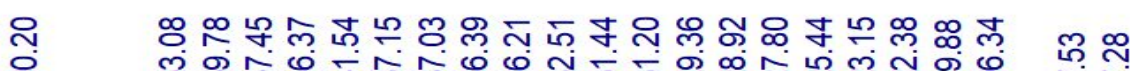

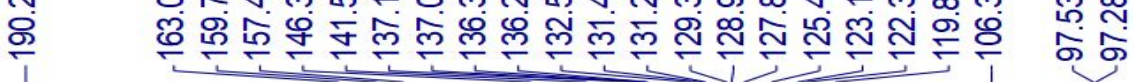

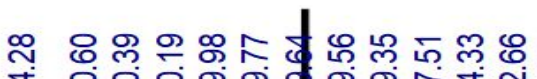

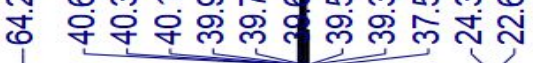
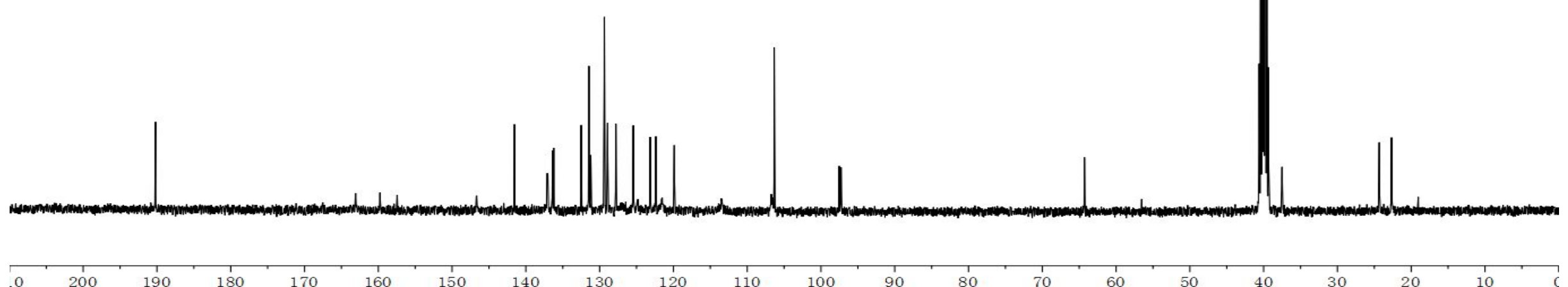

Figure S4. ${ }^{13} \mathrm{C}$ NMR $\left(100 \mathrm{MHz}, \mathrm{DMSO}-d_{6}\right)$ spectra of compound $\mathbf{4 b}$ 


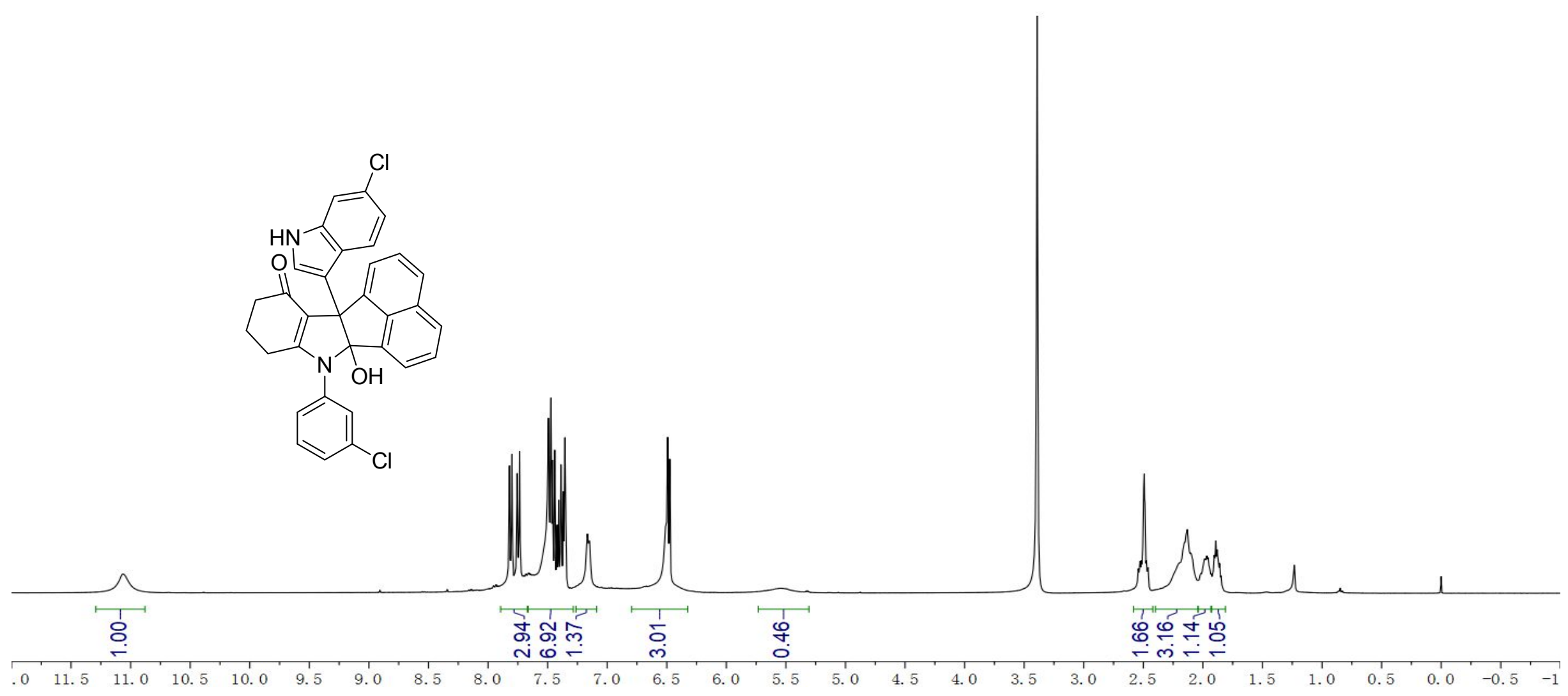

Figure S5. ${ }^{1} \mathrm{H}$ NMR $\left(400 \mathrm{MHz}, \mathrm{DMSO}-d_{6}\right)$ spectra of compound 4c 


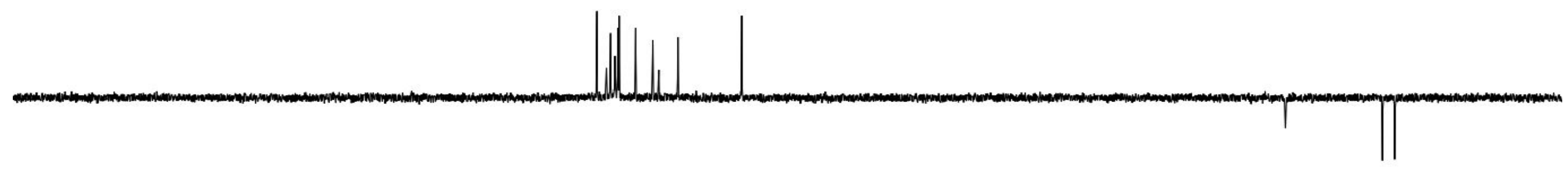

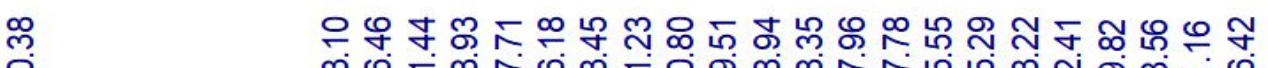

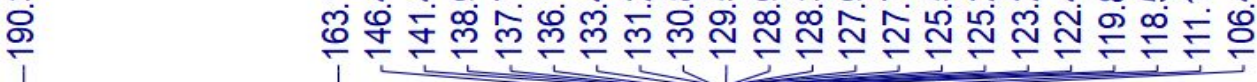

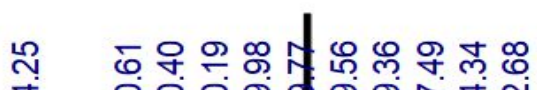

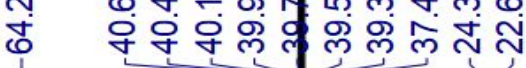

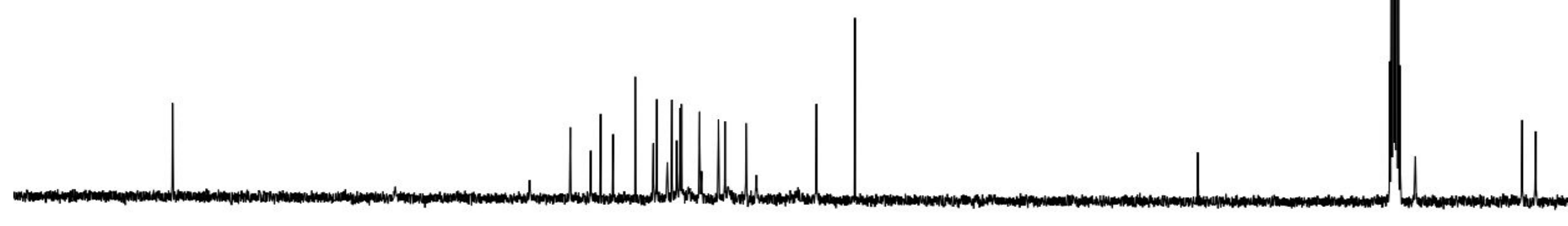

200

$190 \quad 180$

$170 \quad 160$

$150 \quad 140$

$130 \quad 120$

110

so

Figure S6. ${ }^{13} \mathrm{C}$ NMR (100 MHz, DMSO- $\left.d_{6}\right)$ spectra of compound 4c 

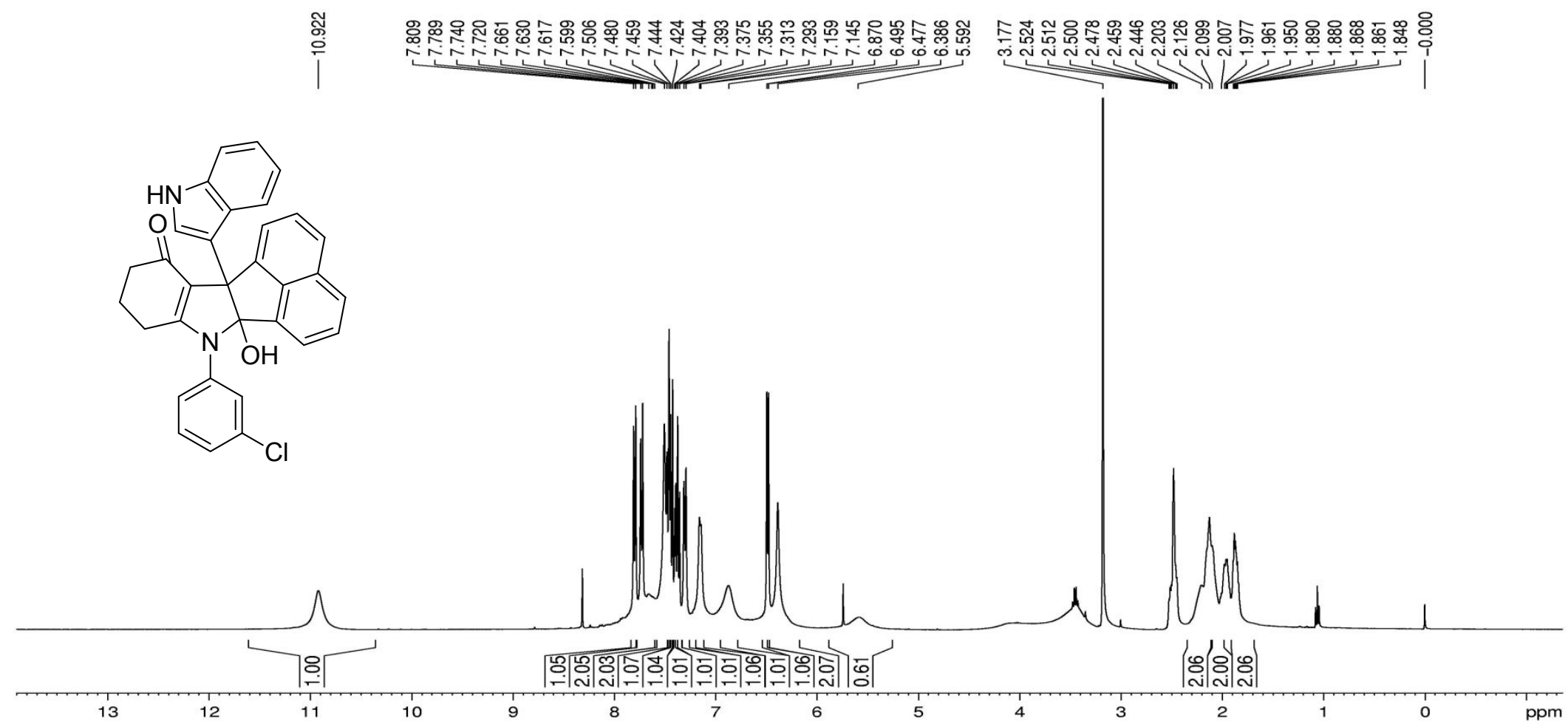

Figure S7. ${ }^{1} \mathrm{H}$ NMR $\left(400 \mathrm{MHz}, \mathrm{DMSO}-d_{6}\right)$ spectra of compound $\mathbf{4 d}$ 


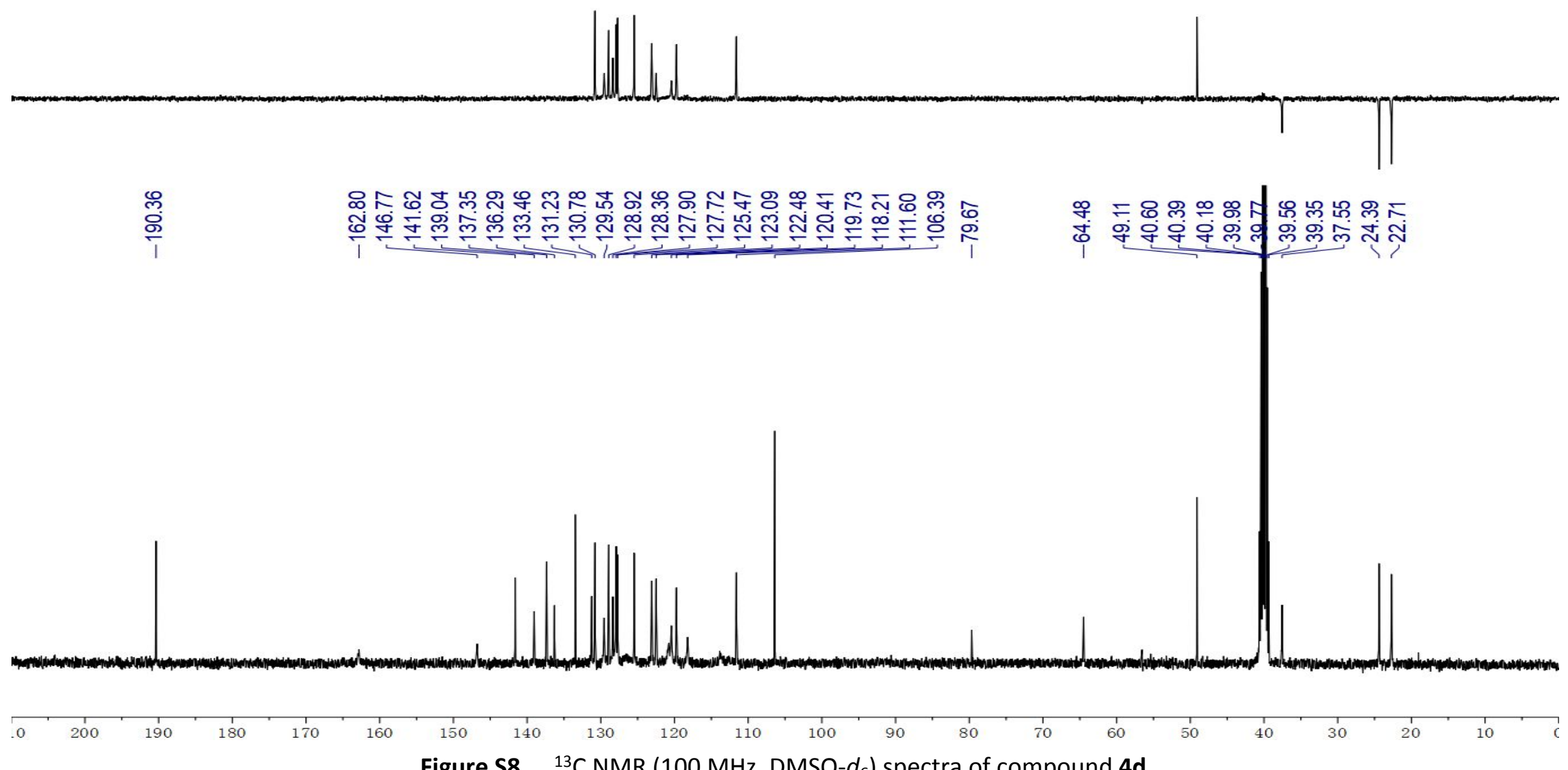

Figure S8. ${ }^{13} \mathrm{C}$ NMR $\left(100 \mathrm{MHz}, \mathrm{DMSO}-d_{6}\right)$ spectra of compound $\mathbf{4 d}$ 


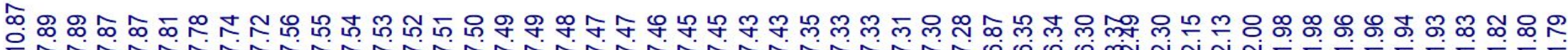

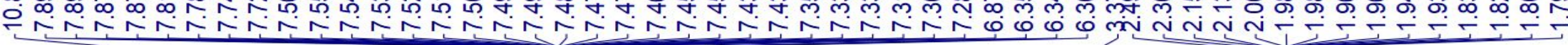

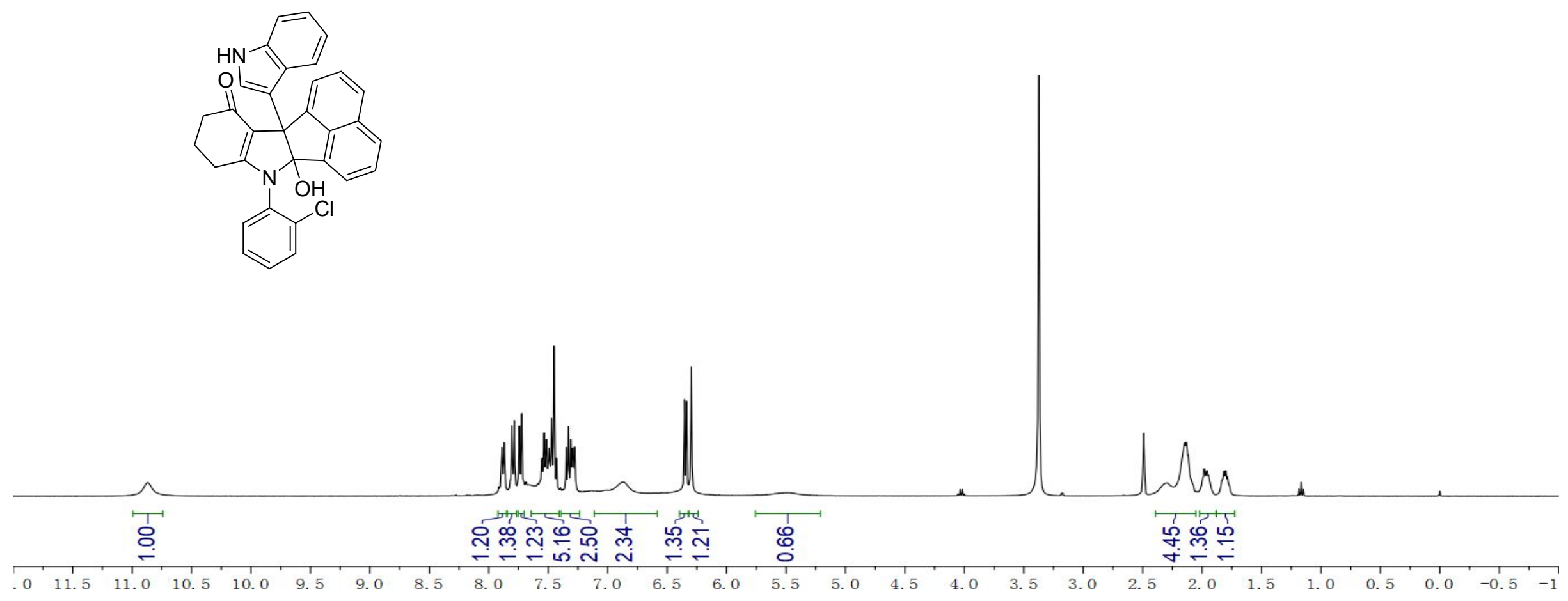

Figure S9. ${ }^{1} \mathrm{H}$ NMR $\left(400 \mathrm{MHz}, \mathrm{DMSO}-d_{6}\right)$ spectra of compound $4 \mathrm{e}$ 

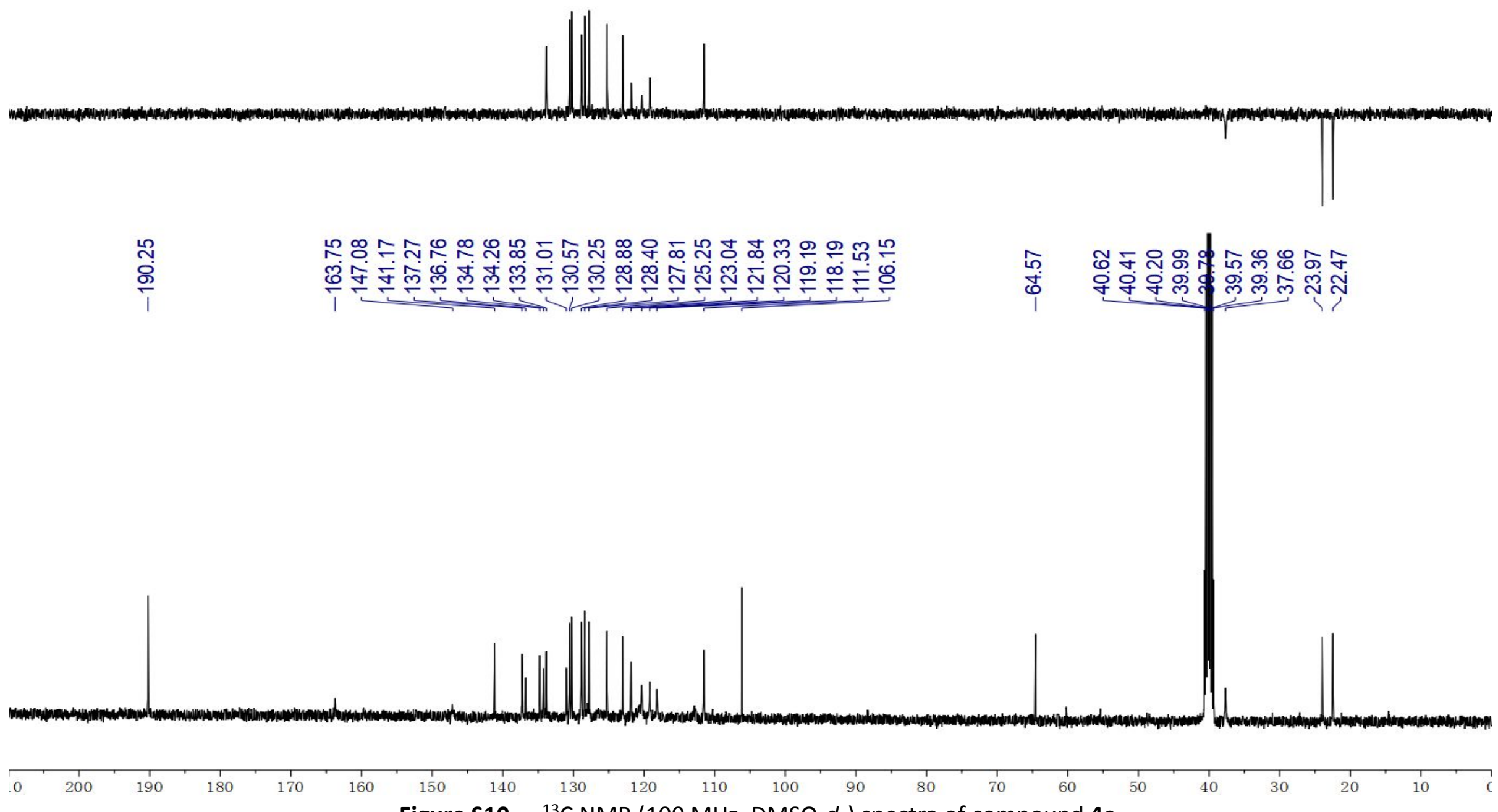

Figure S10. ${ }^{13} \mathrm{C}$ NMR $\left(100 \mathrm{MHz}, \mathrm{DMSO}-d_{6}\right)$ spectra of compound $4 \mathrm{e}$ 


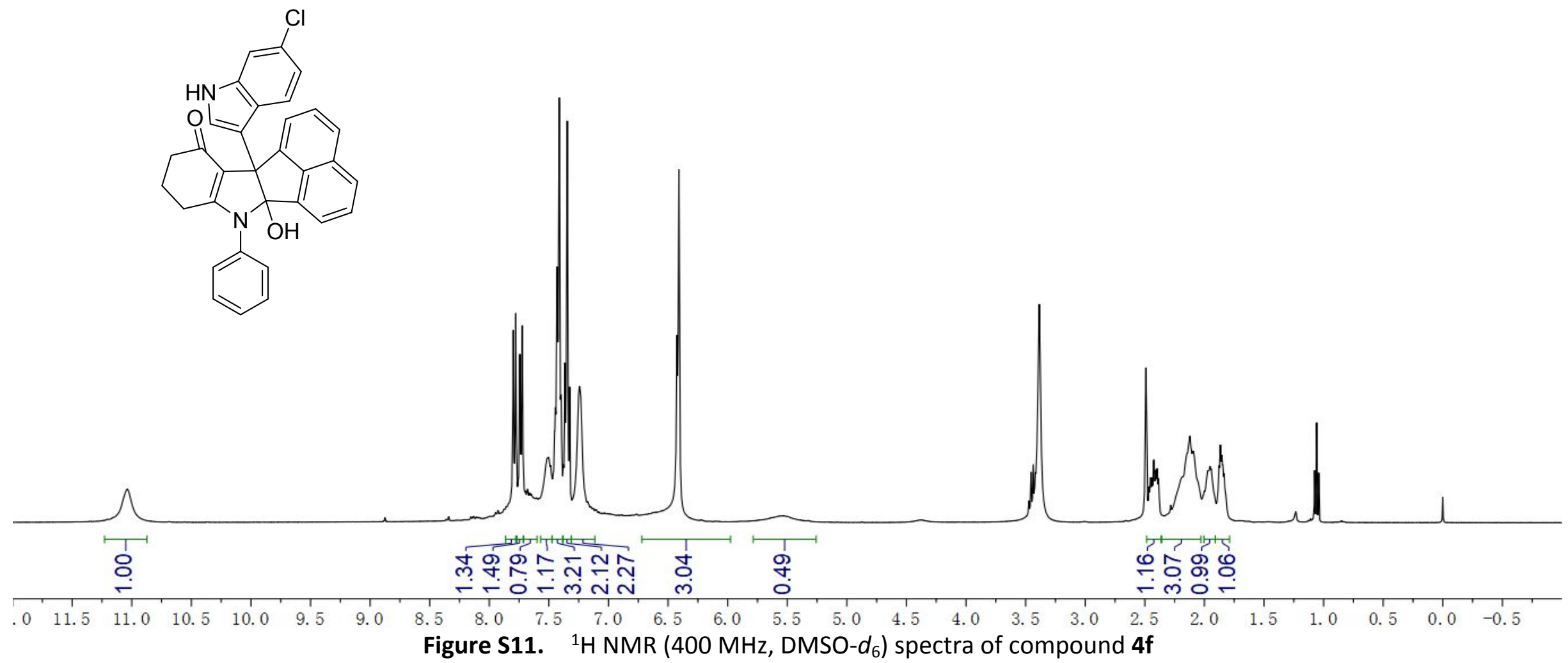




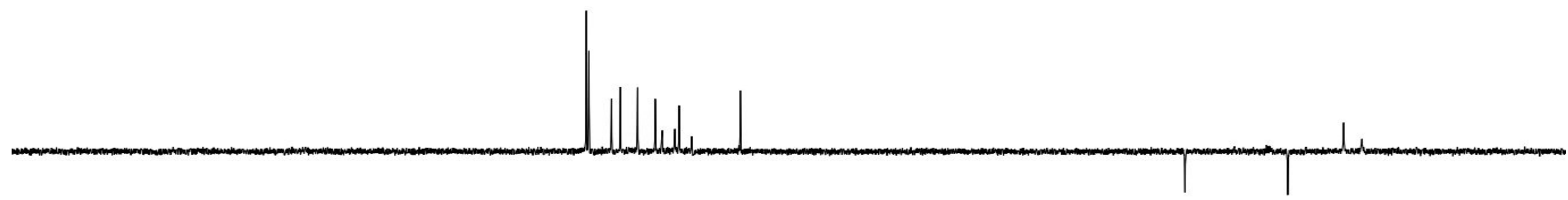

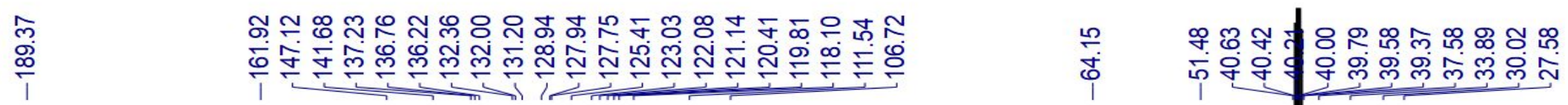
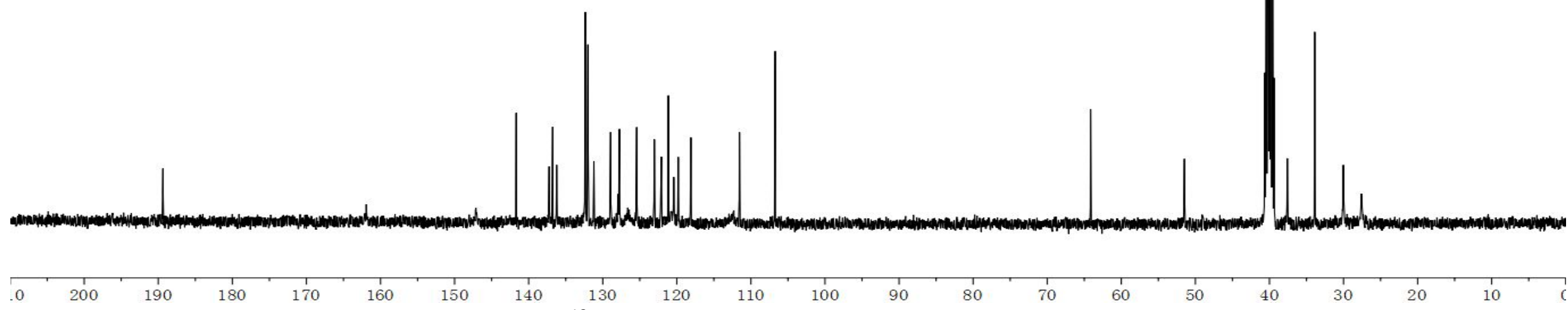

Figure S12. ${ }^{13} \mathrm{C}$ NMR $\left(100 \mathrm{MHz}, \mathrm{DMSO}-d_{6}\right)$ spectra of compound $\mathbf{4 f}$ 
乐
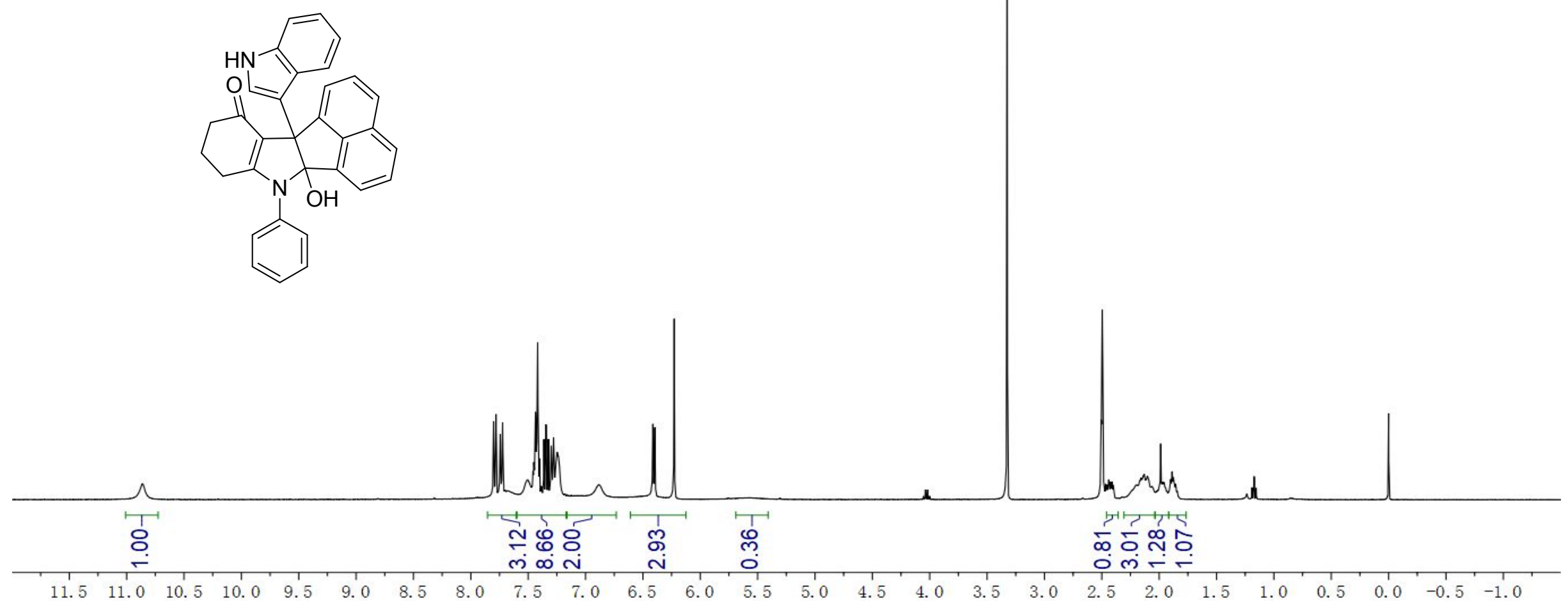

Figure S13. ${ }^{1} \mathrm{H}$ NMR (400 MHz, DMSO- $\left.d_{6}\right)$ spectra of compound $\mathbf{4 g}$ 


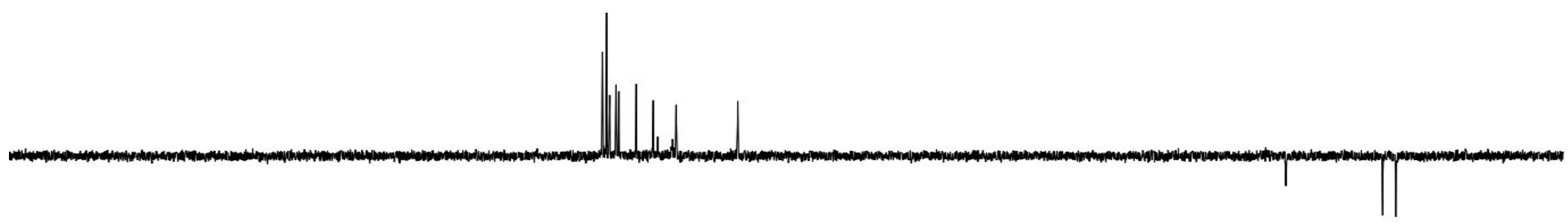

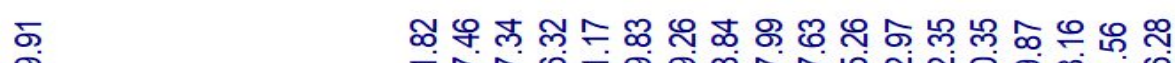

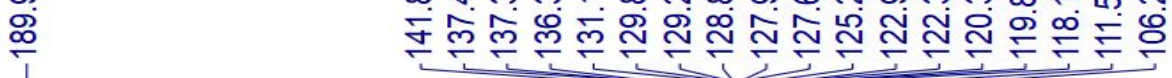

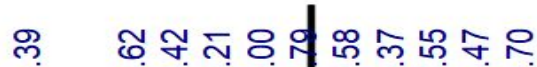

保
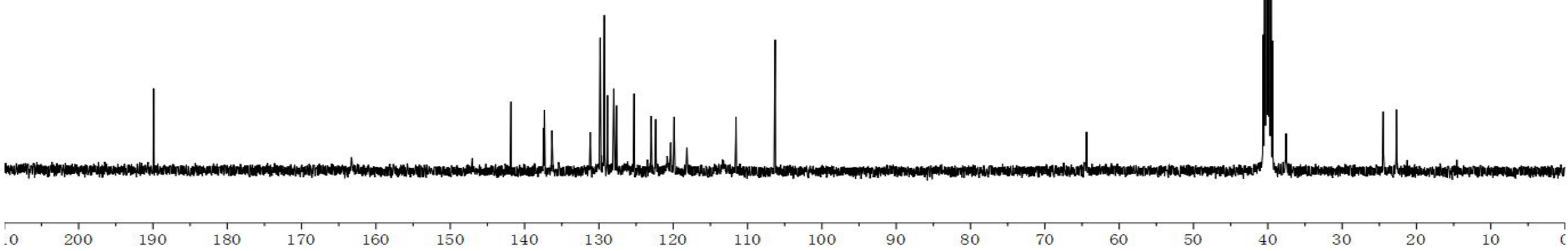

Figure S14. ${ }^{13} \mathrm{C}$ NMR $\left(100 \mathrm{MHz}, \mathrm{DMSO}-d_{6}\right)$ spectra of compound $4 \mathrm{~g}$ 


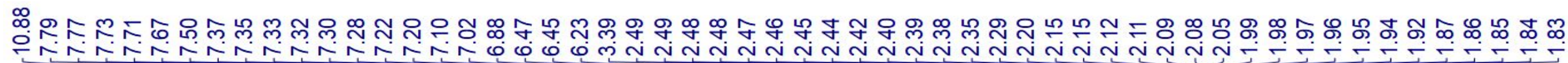

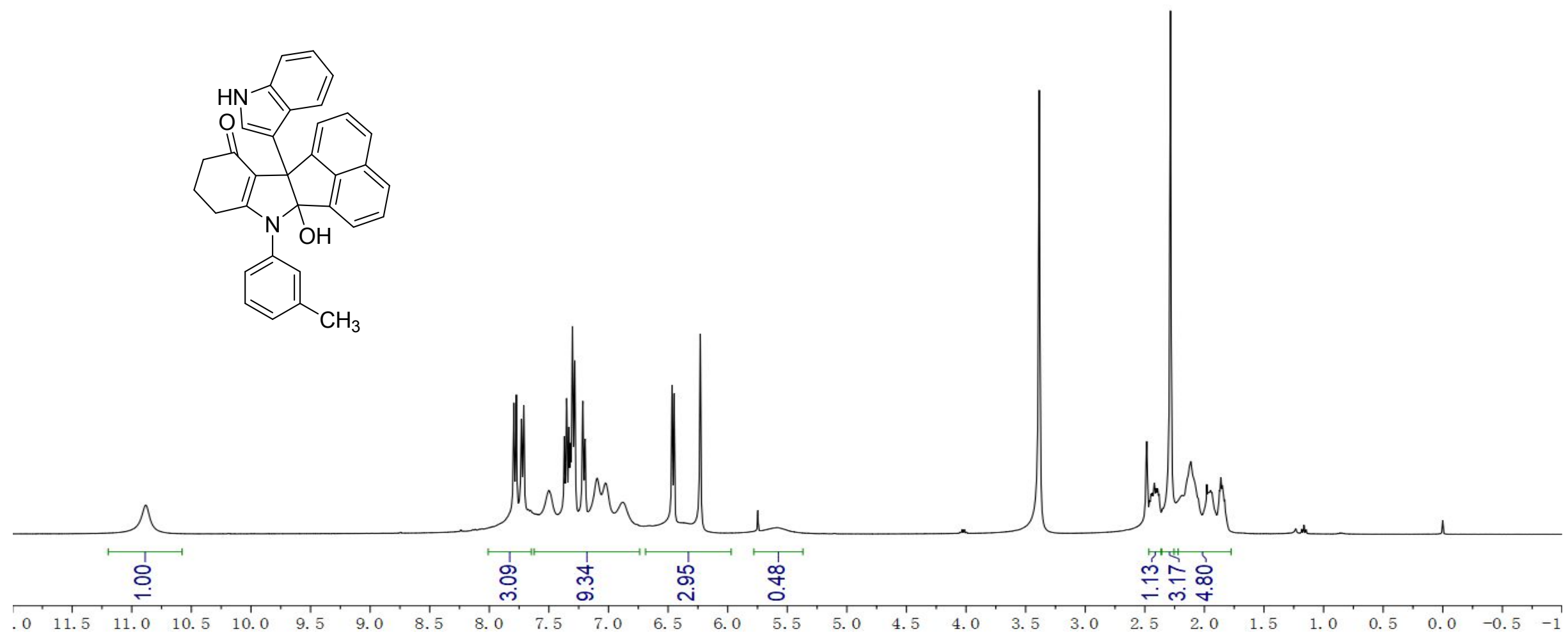

Figure S15. ${ }^{1} \mathrm{H}$ NMR (400 MHz, DMSO- $d_{6}$ ) spectra of compound $4 \mathrm{~h}$ 


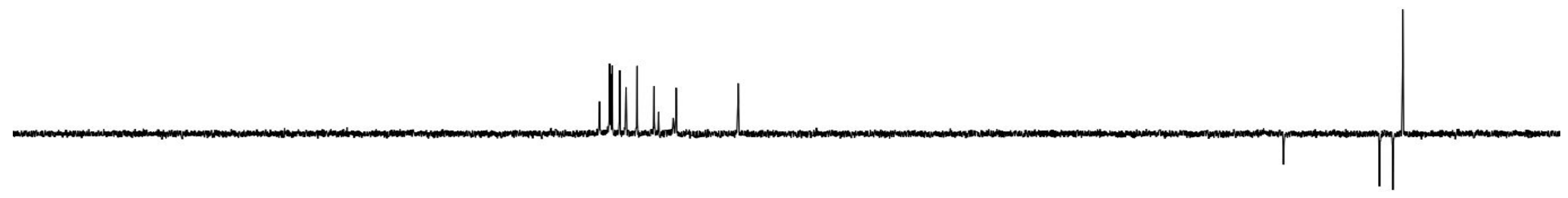

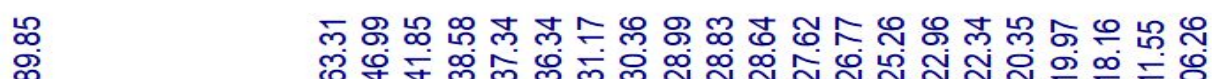

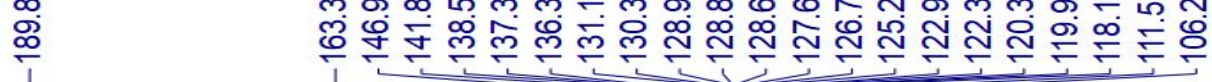

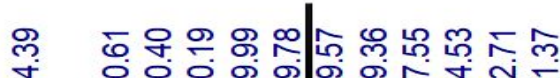

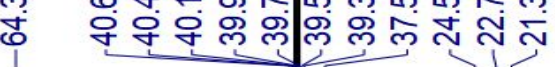

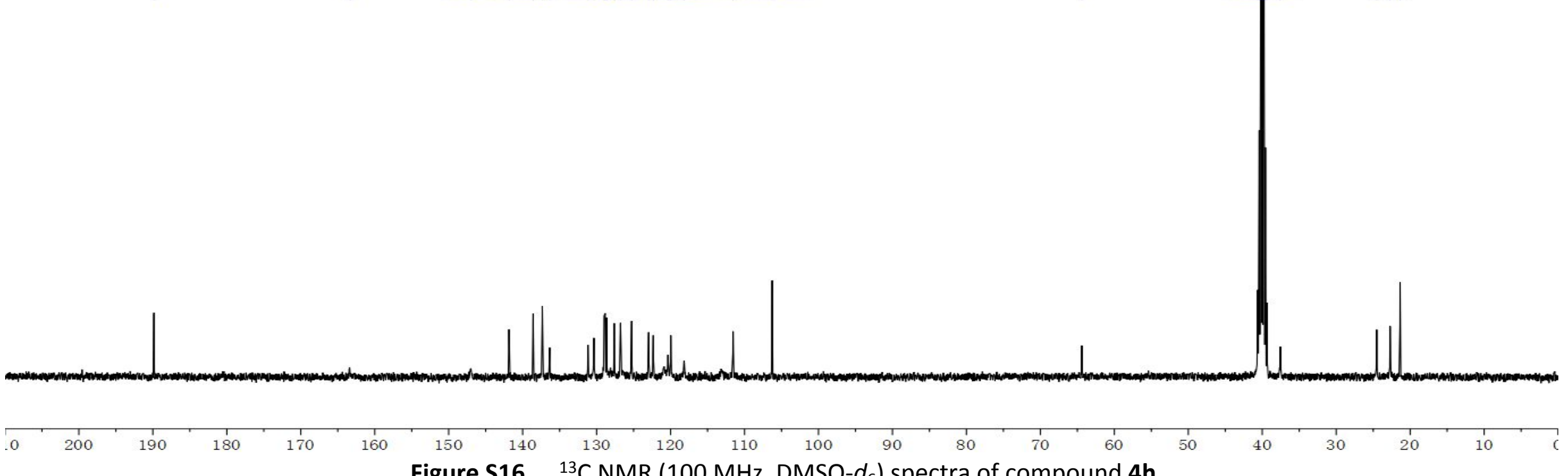

Figure S16. ${ }^{13} \mathrm{C}$ NMR (100 MHz, DMSO- $\left.d_{6}\right)$ spectra of compound $\mathbf{4 h}$ 

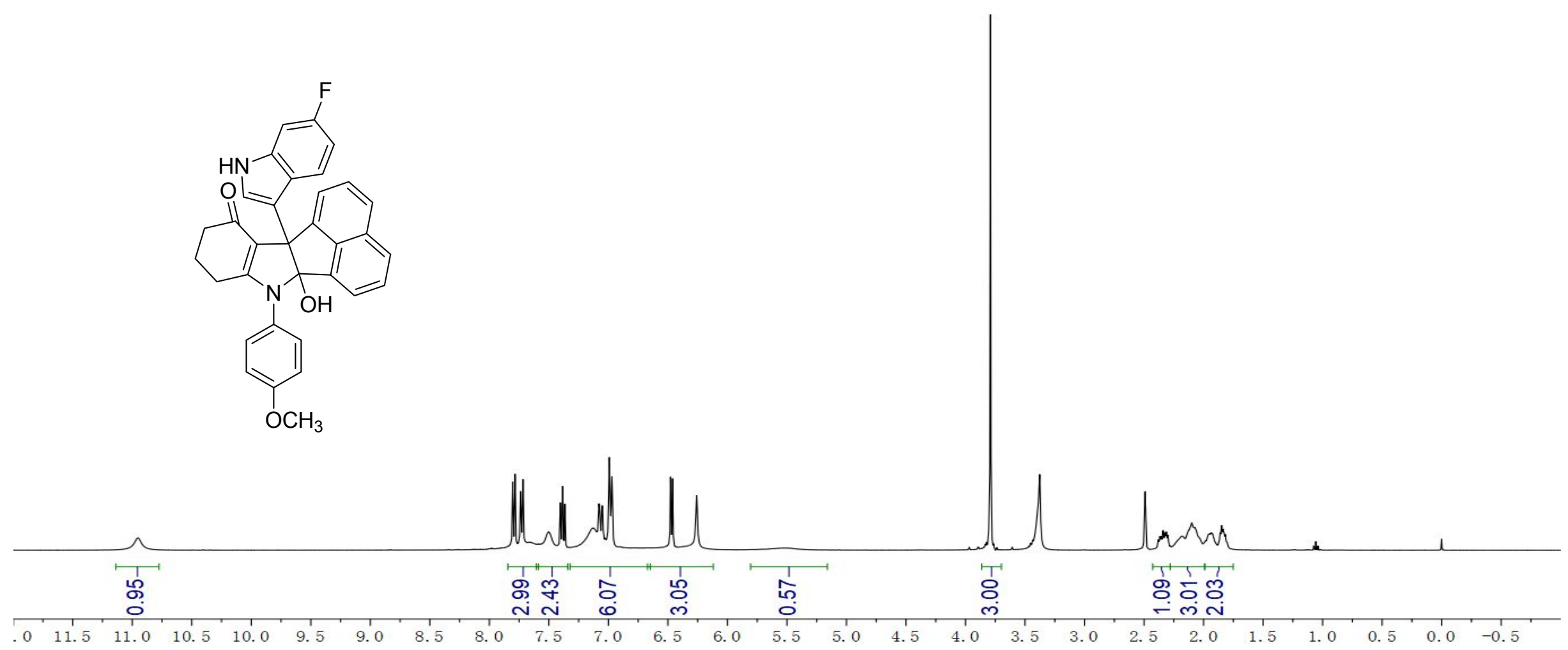

Figure S17. ${ }^{1} \mathrm{H}$ NMR $\left(400 \mathrm{MHz}, \mathrm{DMSO}-d_{6}\right)$ spectra of compound $4 \mathbf{i}$ 


$$
=\mathrm{T}
$$




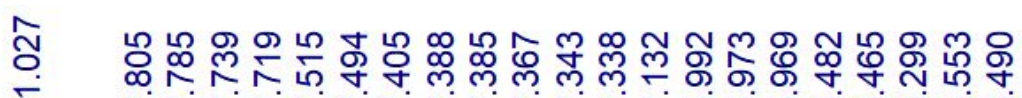

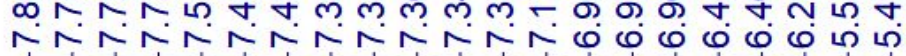

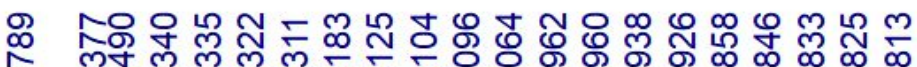

min mind

I MNNNNNNNNNTTTTT

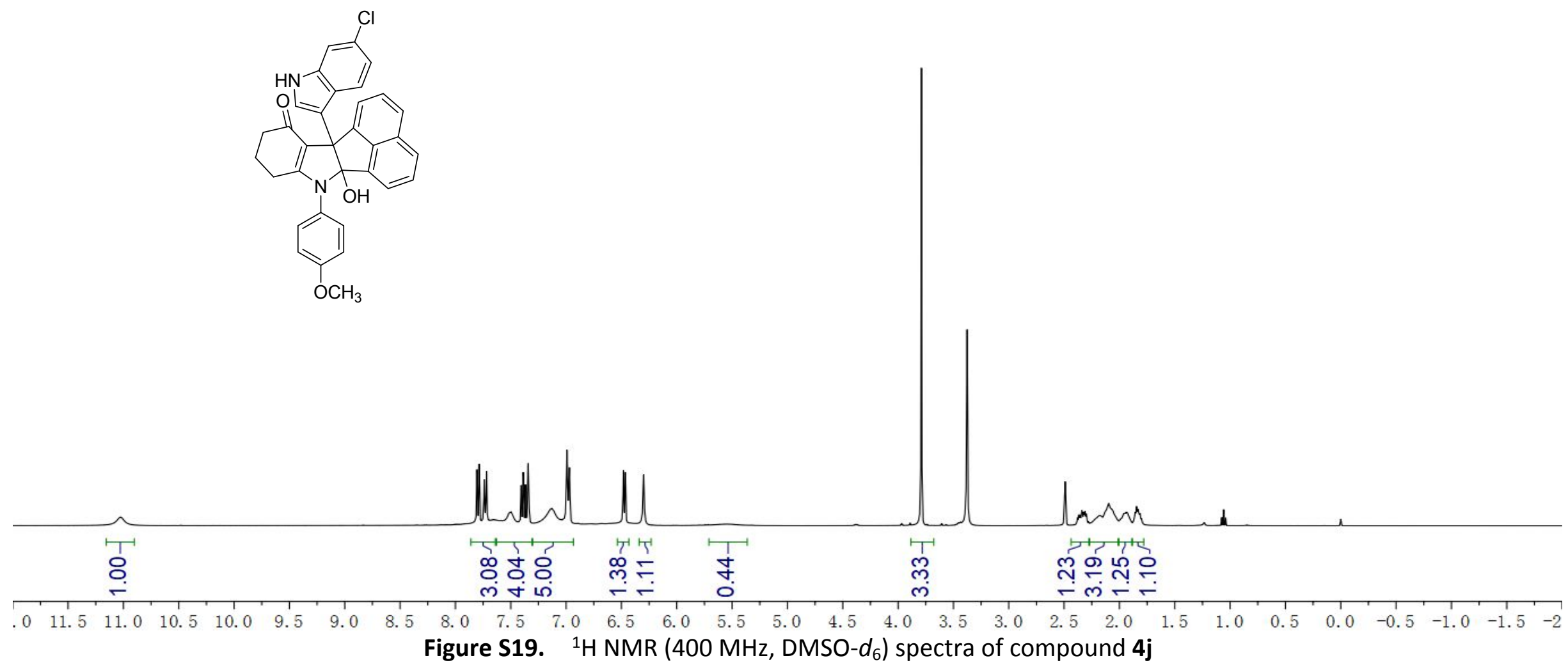



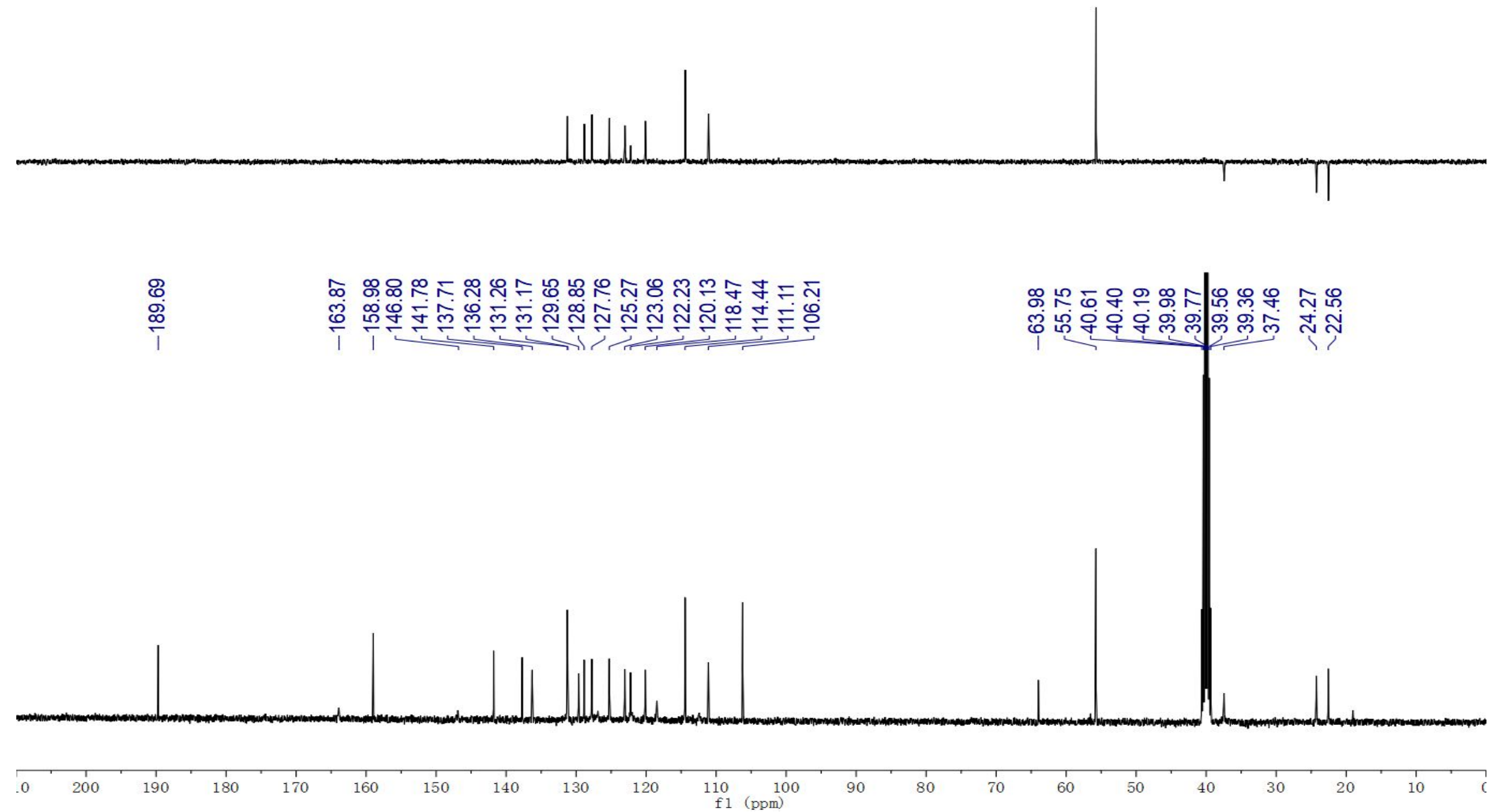

Figure S20. ${ }^{13} \mathrm{C}$ NMR (100 MHz, DMSO- $\left.d_{6}\right)$ spectra of compound $4 \mathbf{j}$ 


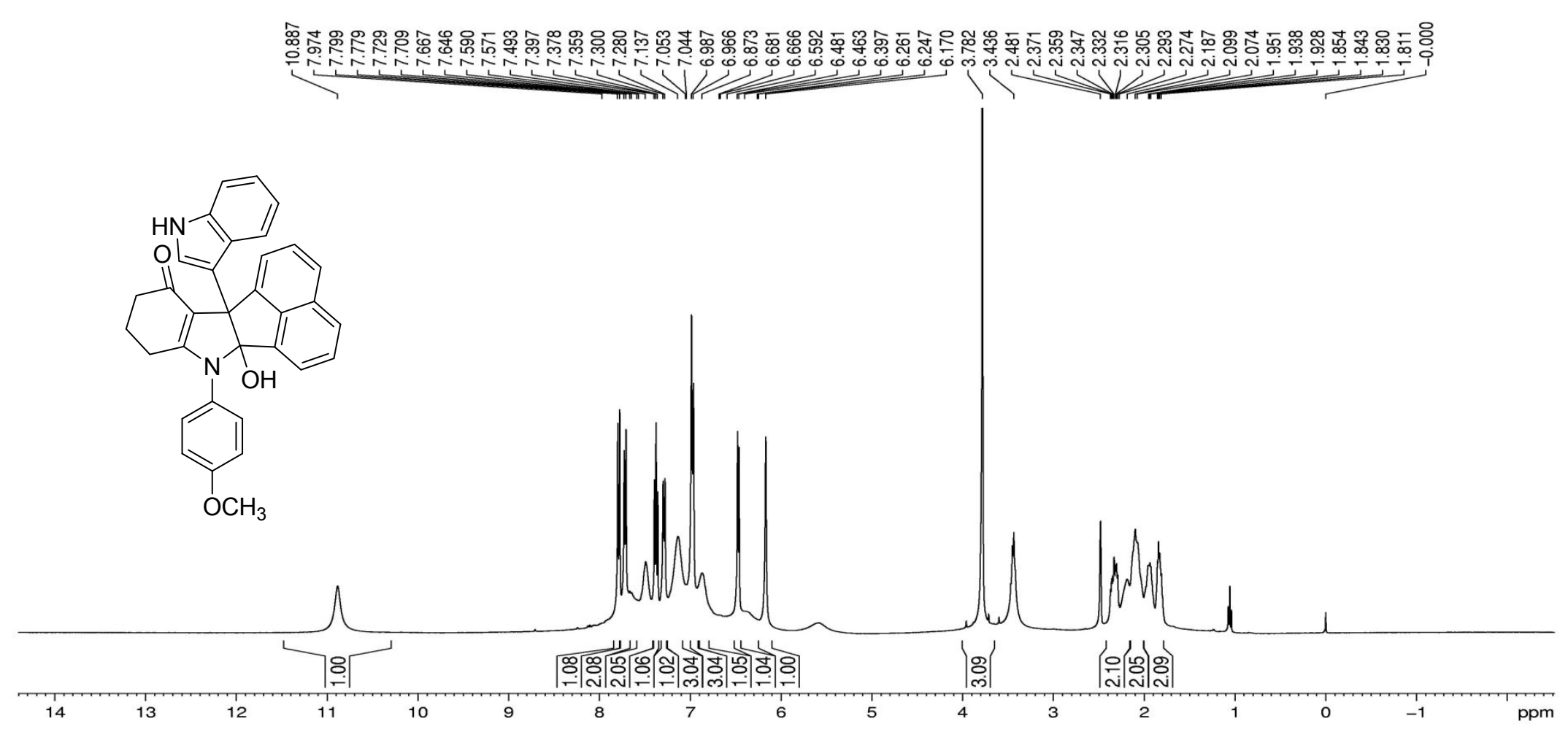

Figure S21. ${ }^{1} \mathrm{H}$ NMR $\left(400 \mathrm{MHz}, \mathrm{DMSO}-d_{6}\right)$ spectra of compound $\mathbf{4 k}$ 


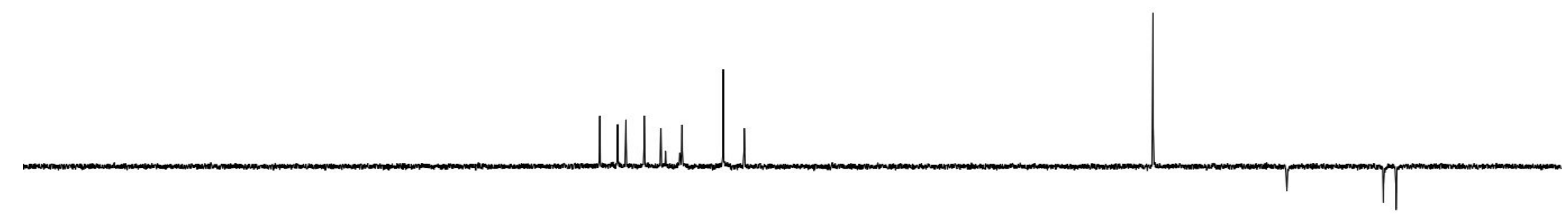

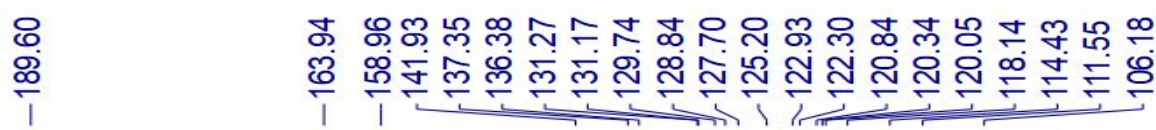

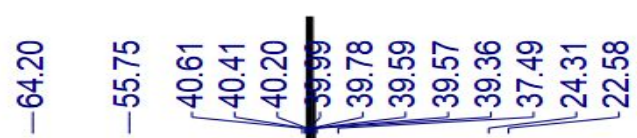

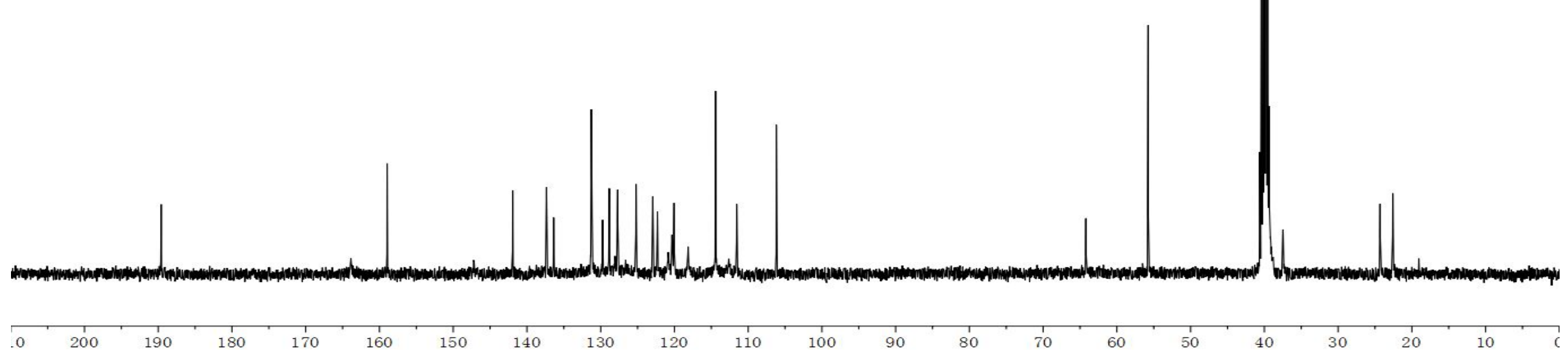

Figure S22. ${ }^{13} \mathrm{C}$ NMR (100 MHz, DMSO- $\left.d_{6}\right)$ spectra of compound $\mathbf{4 k}$ 


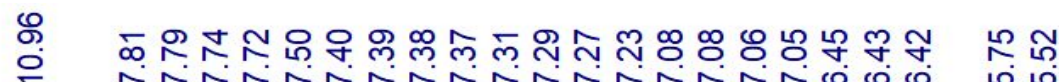

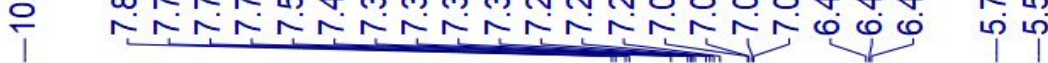

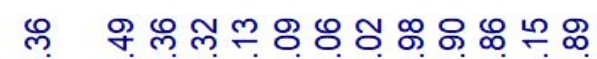
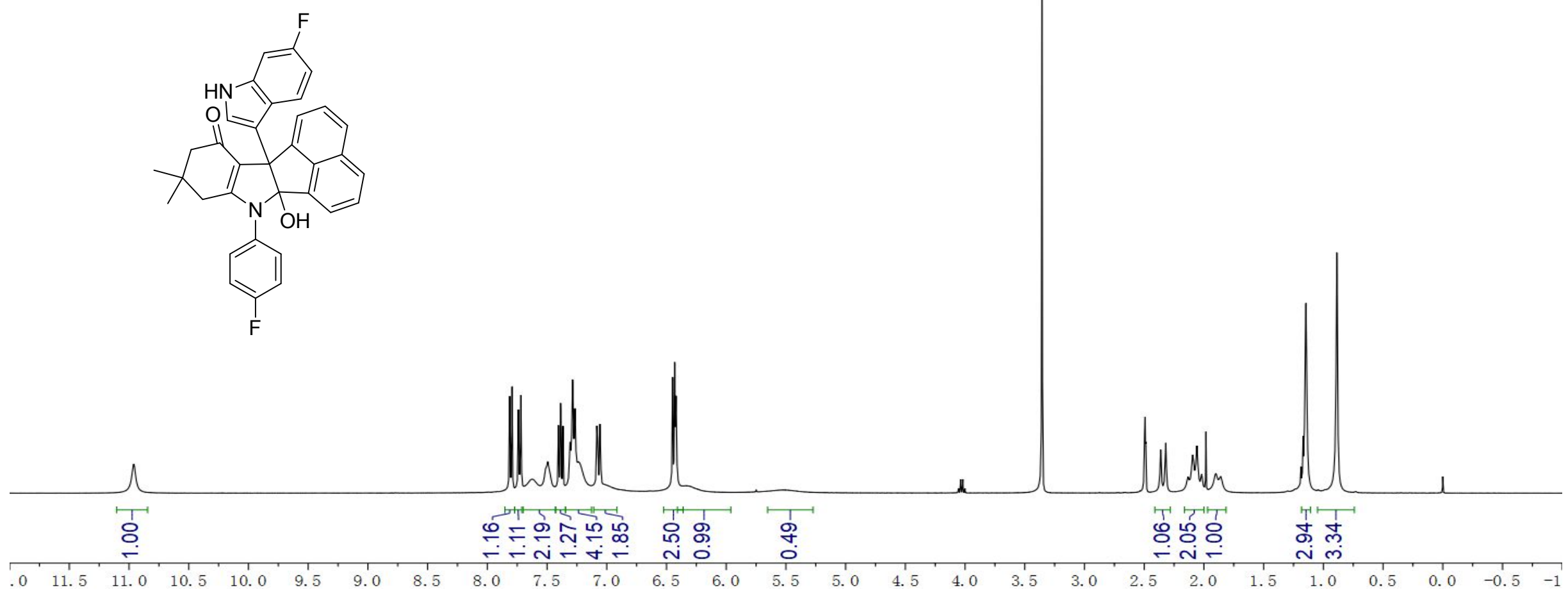

Figure S23. ${ }^{1} \mathrm{H}$ NMR $\left(400 \mathrm{MHz}, \mathrm{DMSO}-d_{6}\right)$ spectra of compound $4 \mathrm{I}$ 


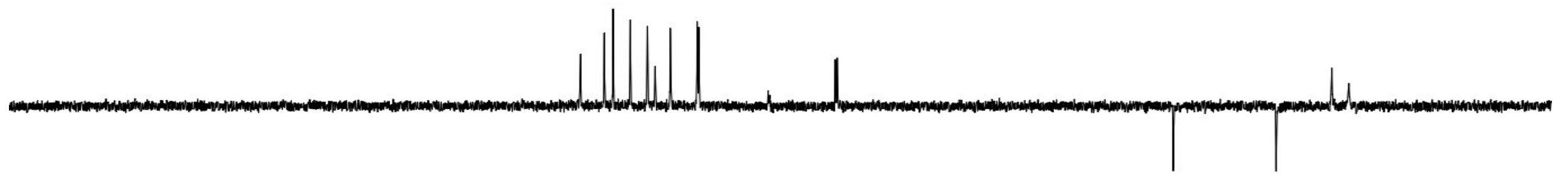

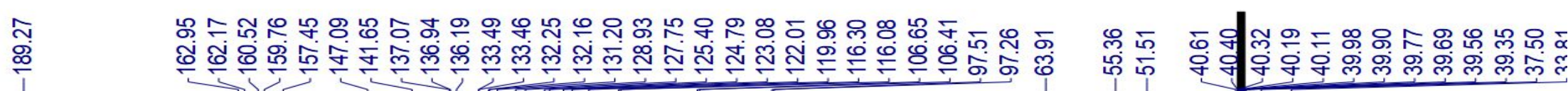
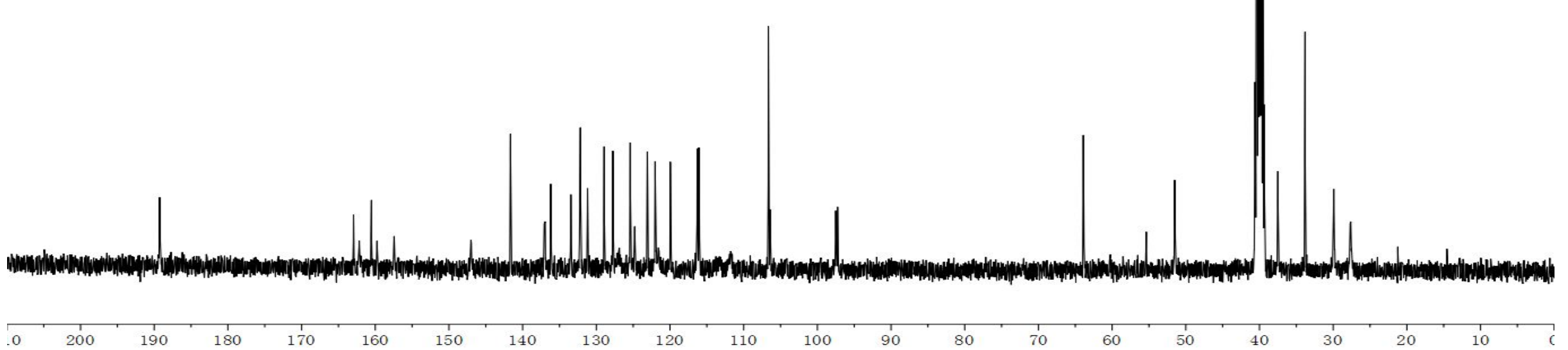

Figure S24. ${ }^{13} \mathrm{C}$ NMR (100 MHz, DMSO- $\left.d_{6}\right)$ spectra of compound 4I 


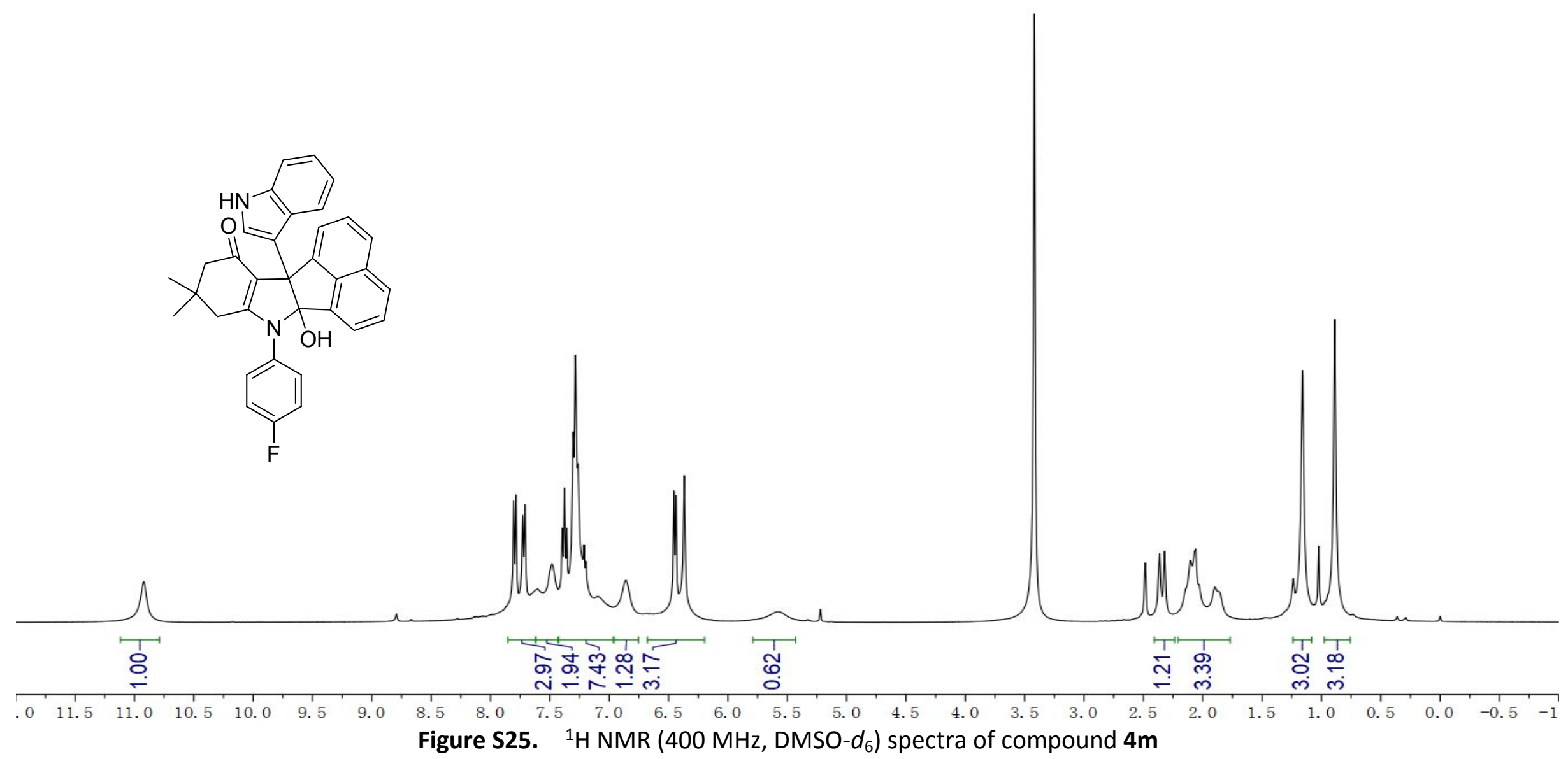




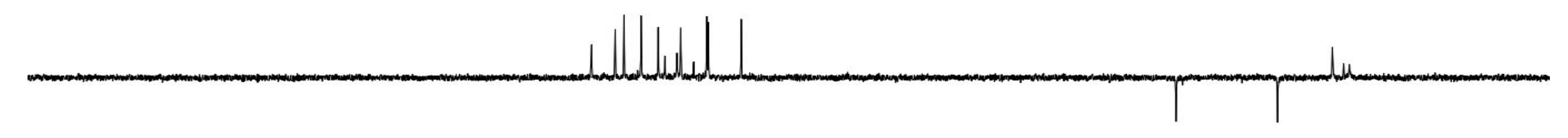

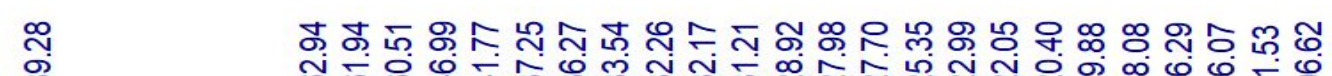

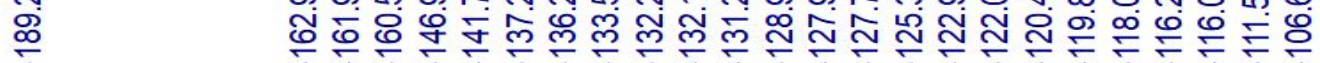

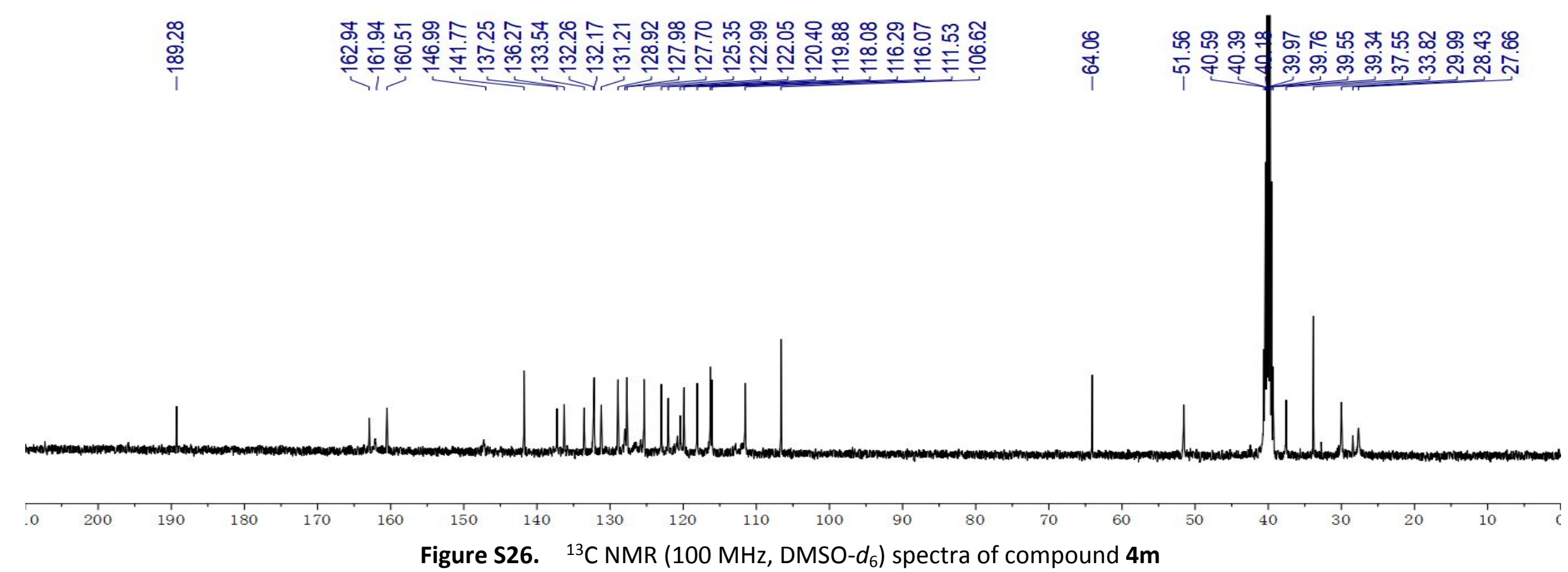




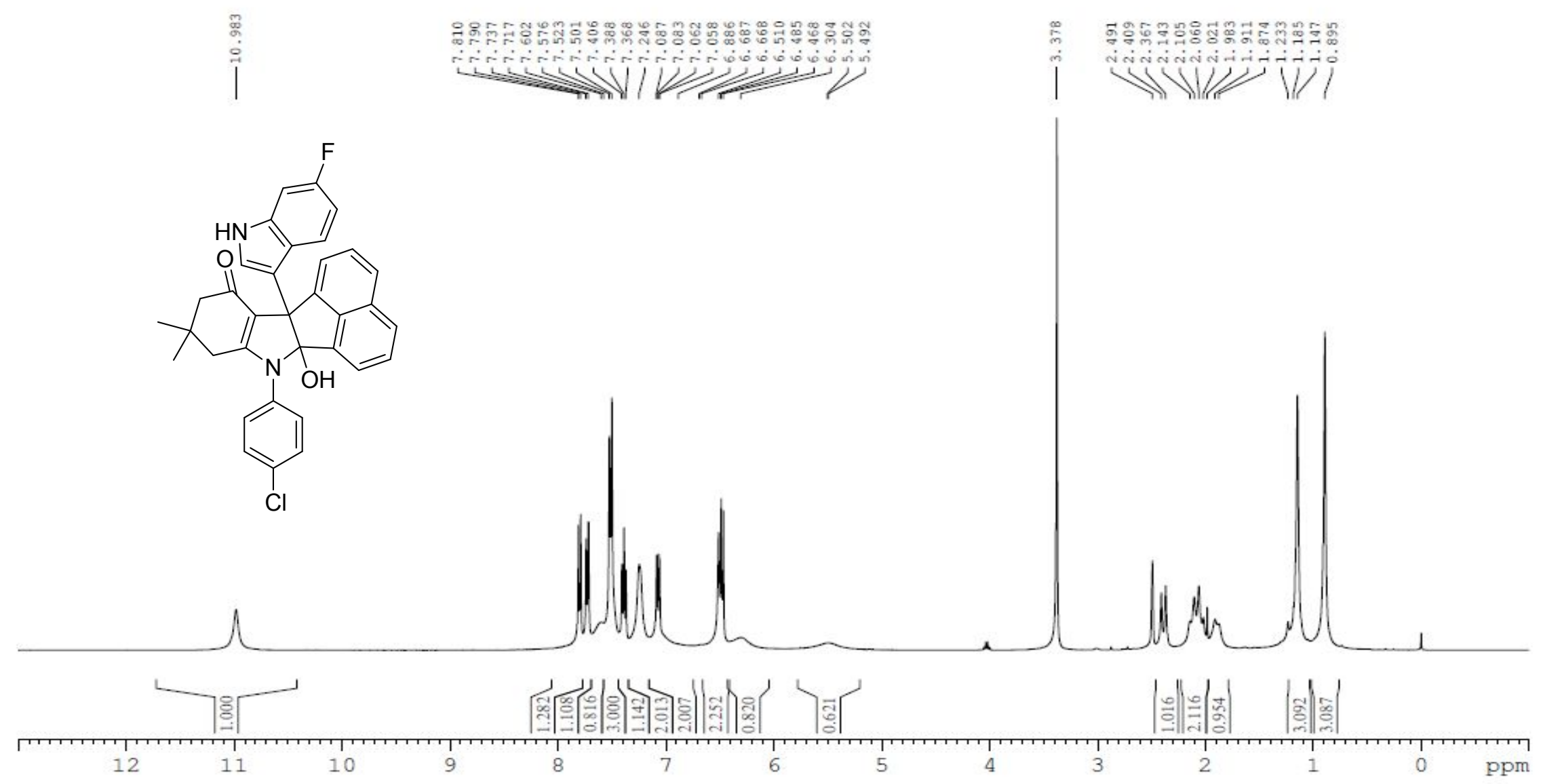

Figure S27. ${ }^{1} \mathrm{H}$ NMR $\left(400 \mathrm{MHz}, \mathrm{DMSO}-d_{6}\right)$ spectra of compound $4 \mathbf{n}$ 

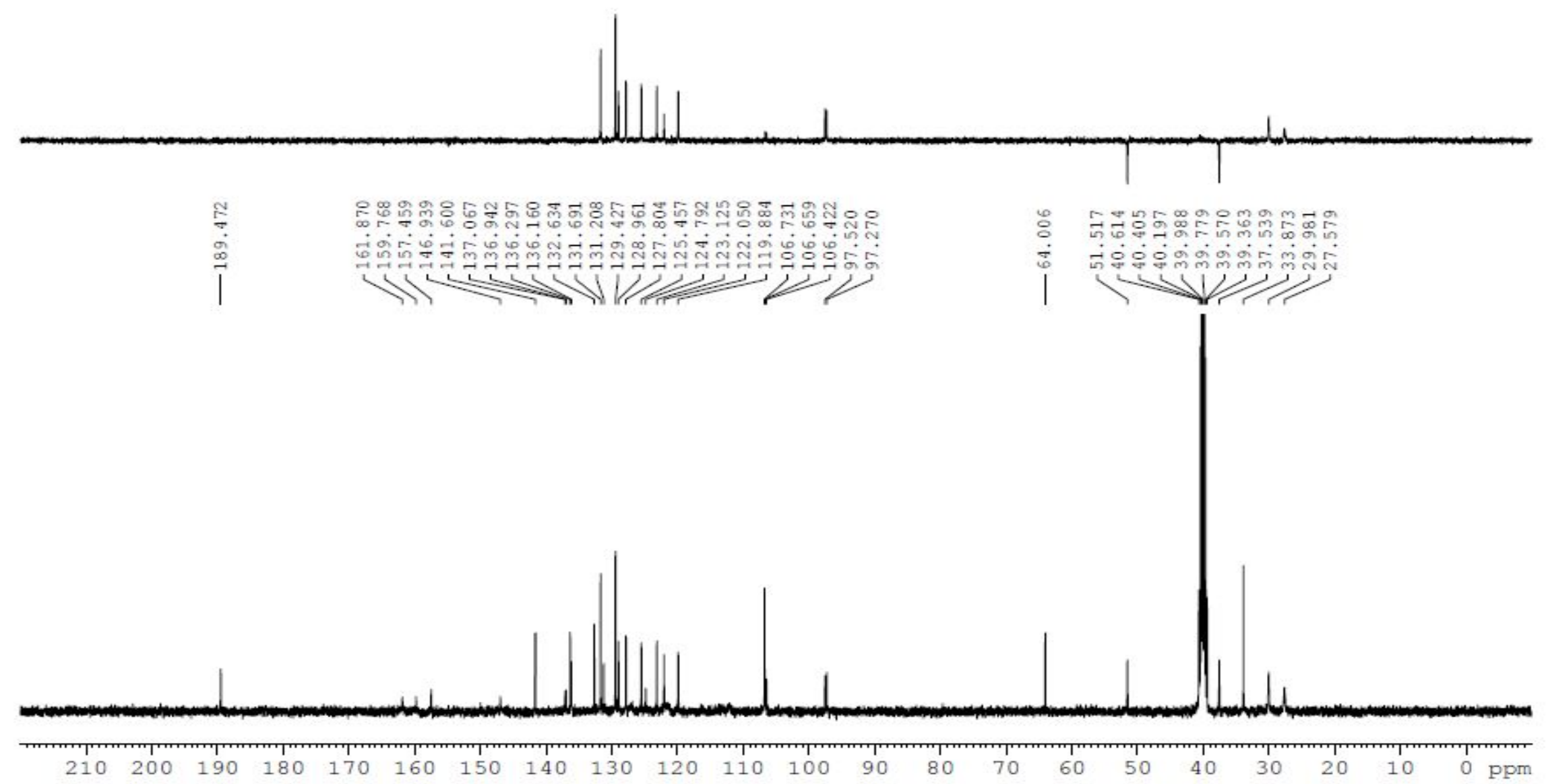

Figure S28. ${ }^{13} \mathrm{C}$ NMR $\left(100 \mathrm{MHz}, \mathrm{DMSO}-d_{6}\right)$ spectra of compound $4 \mathrm{n}$ 


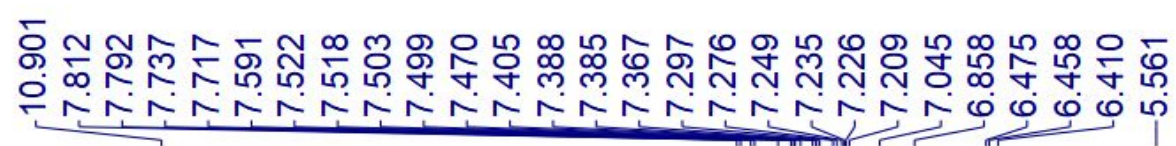

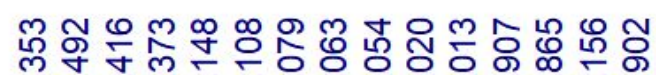
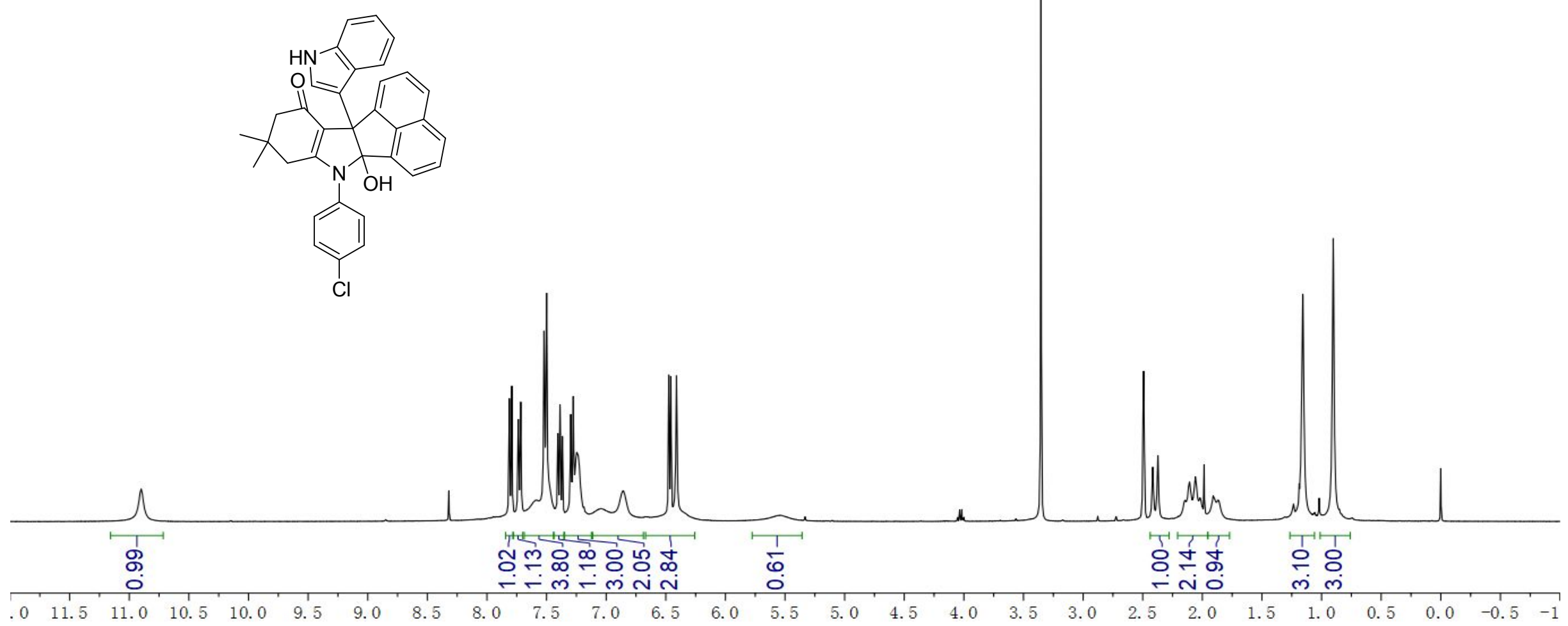

Figure S29. ${ }^{1} \mathrm{H}$ NMR (400 MHz, DMSO- $\left.d_{6}\right)$ spectra of compound 40 

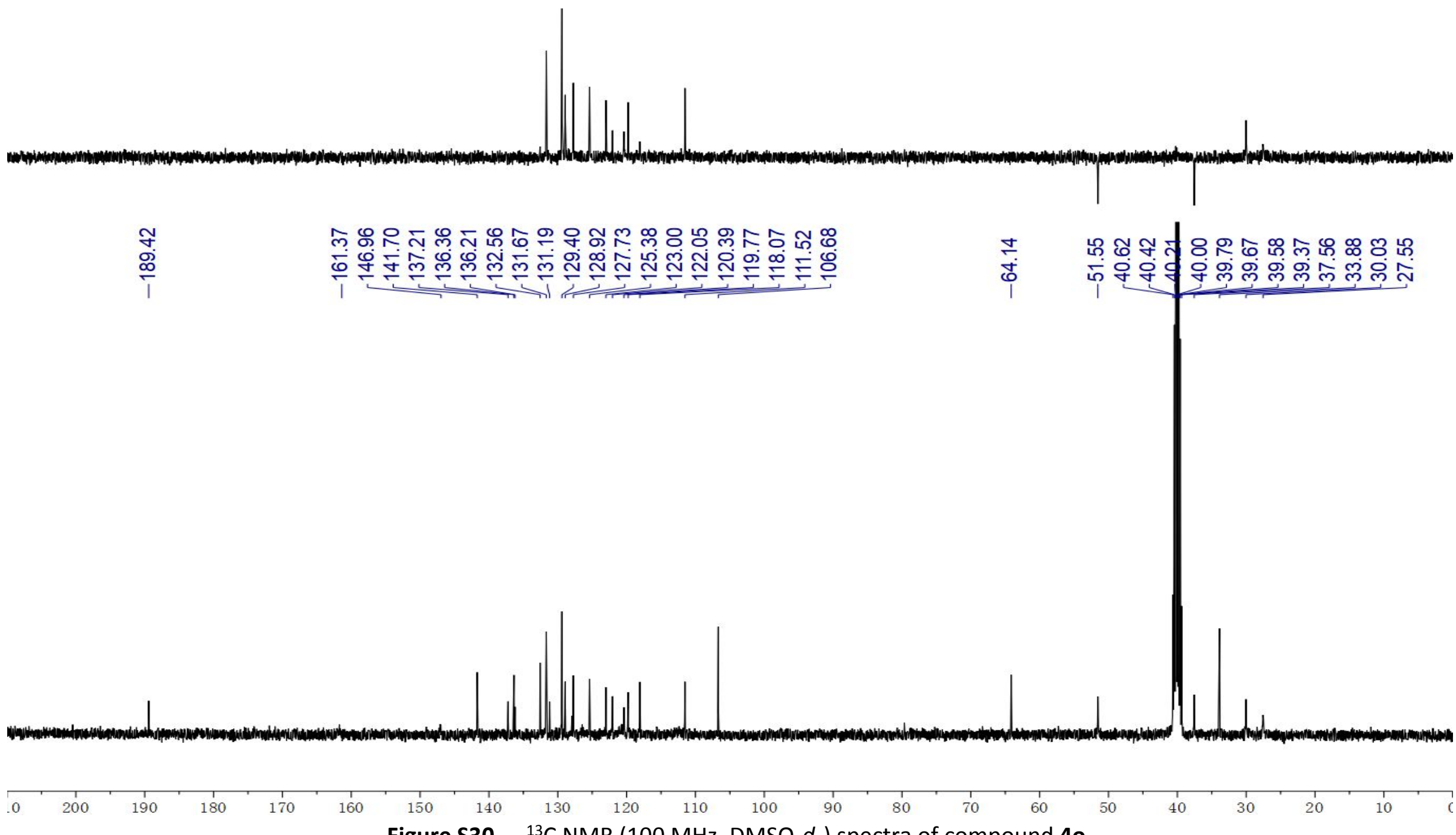

Figure S30. ${ }^{13} \mathrm{C}$ NMR (100 MHz, DMSO- $\left.d_{6}\right)$ spectra of compound 40 


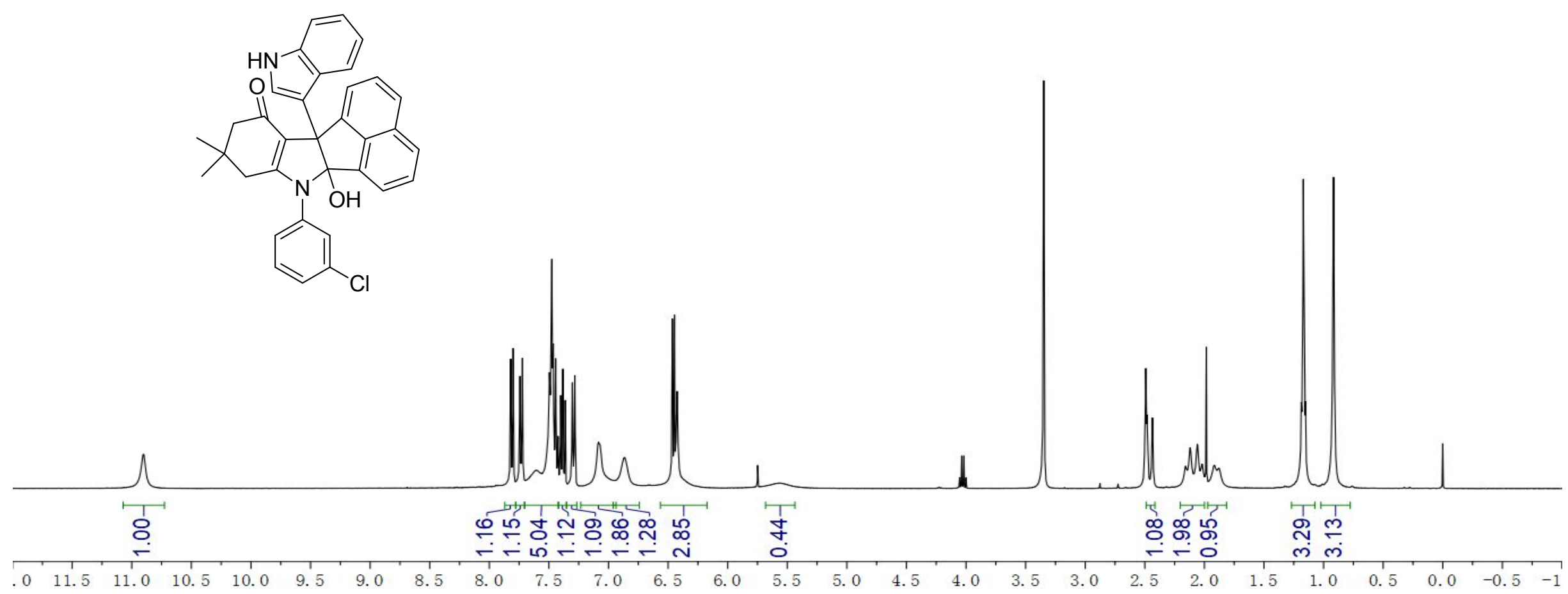

Figure S31. ${ }^{1} \mathrm{H}$ NMR (400 MHz, DMSO- $\left.d_{6}\right)$ spectra of compound $4 p$ 

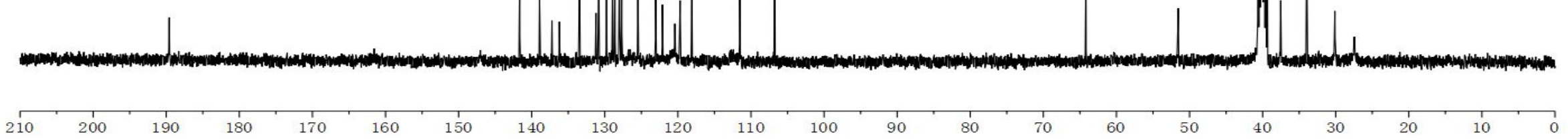

Figure S32. ${ }^{13} \mathrm{C}$ NMR (100 MHz, DMSO- $\left.d_{6}\right)$ spectra of compound $4 p$ 


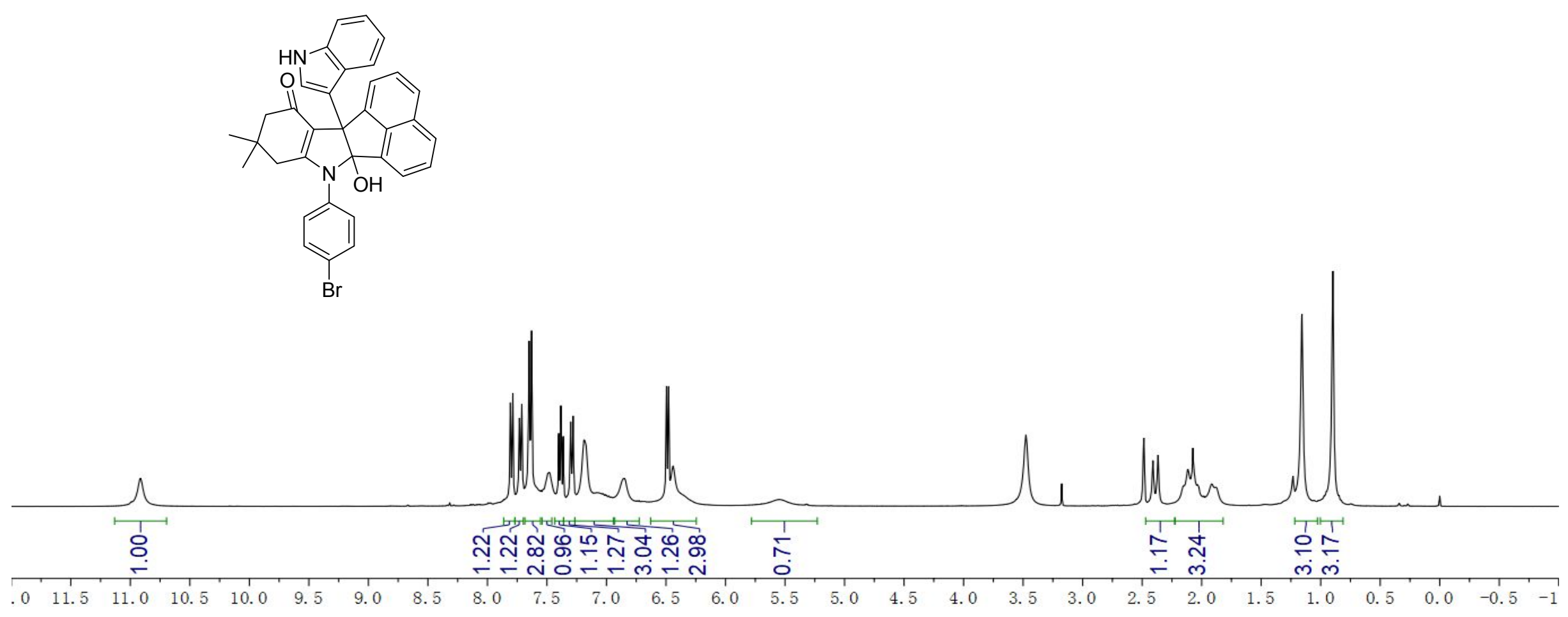

Figure S33. ${ }^{1} \mathrm{H}$ NMR (400 MHz, DMSO- $\left.d_{6}\right)$ spectra of compound $\mathbf{4 q}$ 


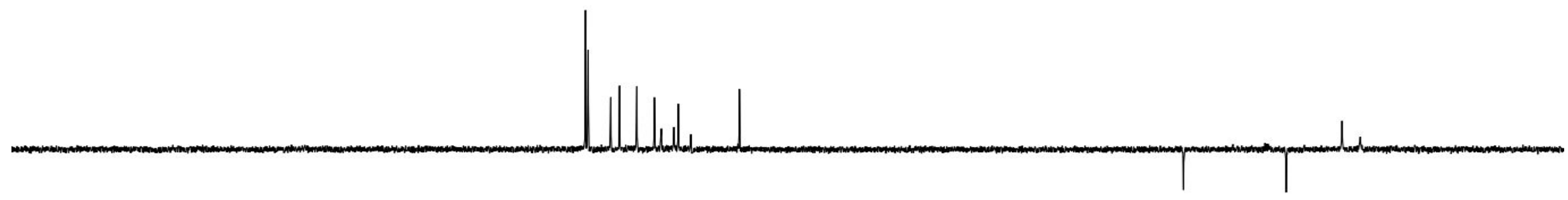

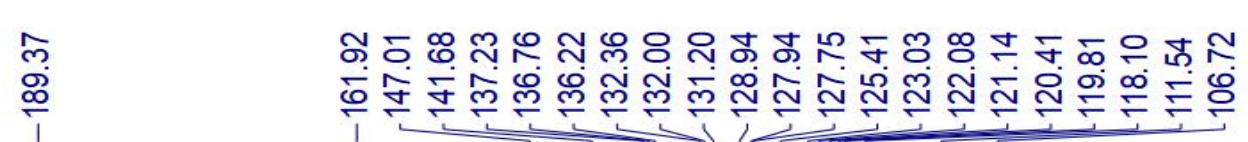

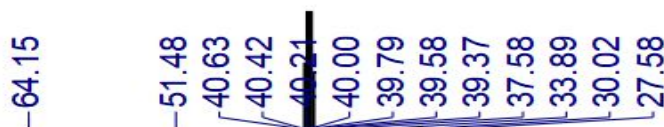

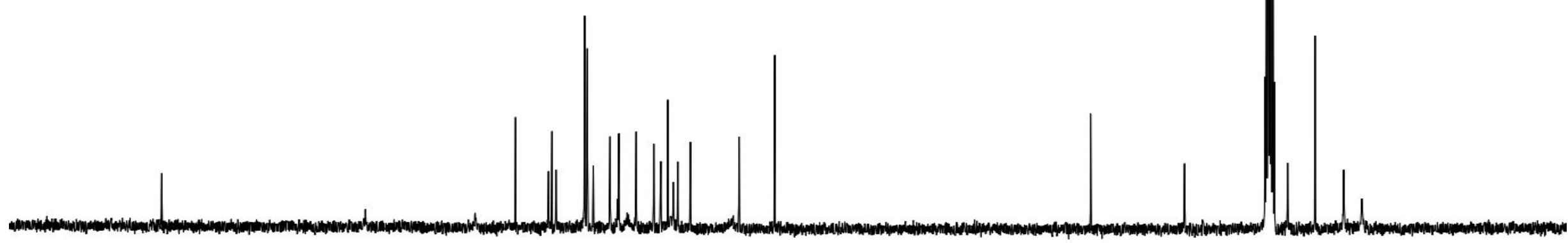

Figure S34. ${ }^{13} \mathrm{C}$ NMR (100 MHz, DMSO- $\left.d_{6}\right)$ spectra of compound $4 q$ 


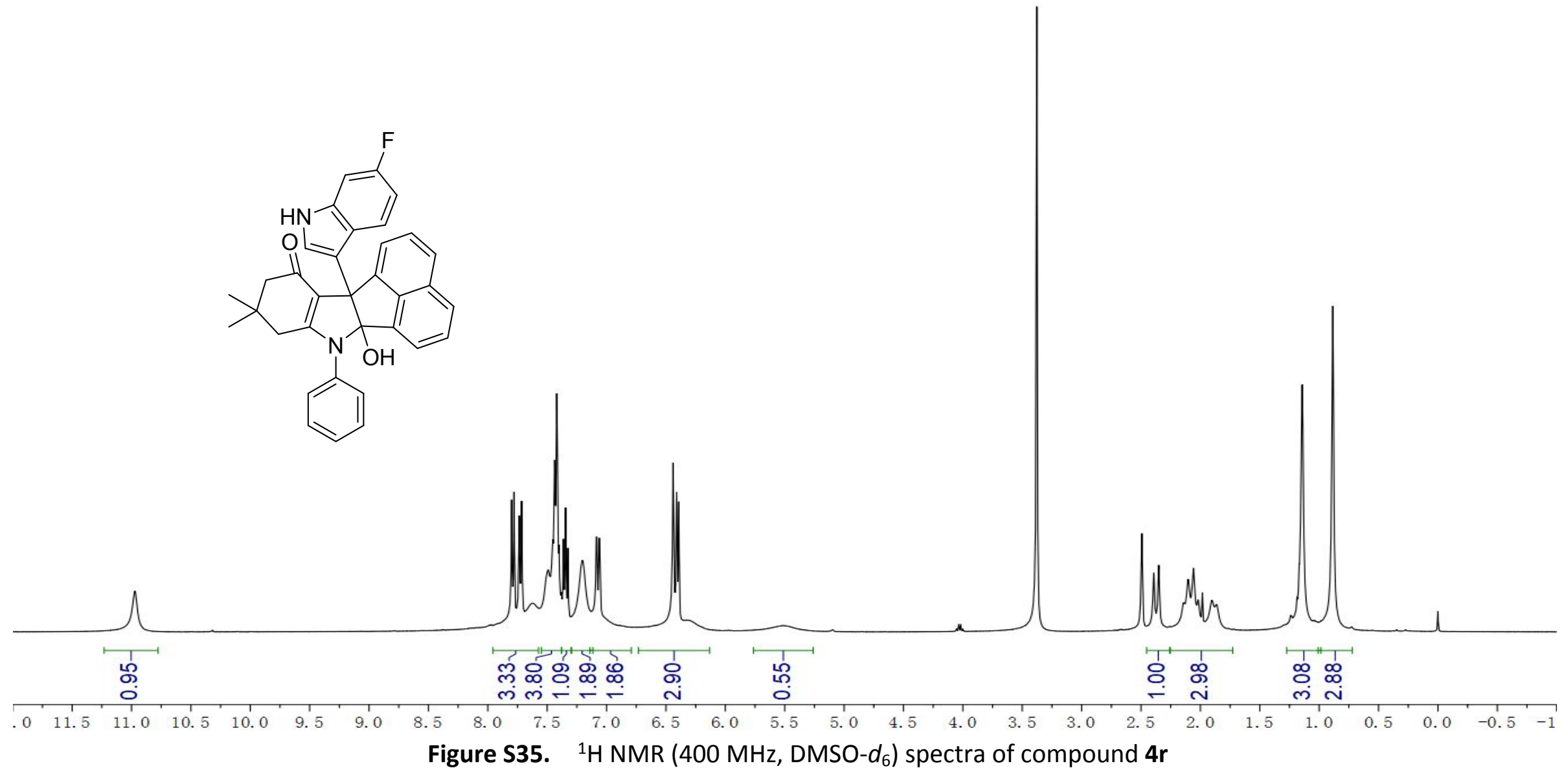




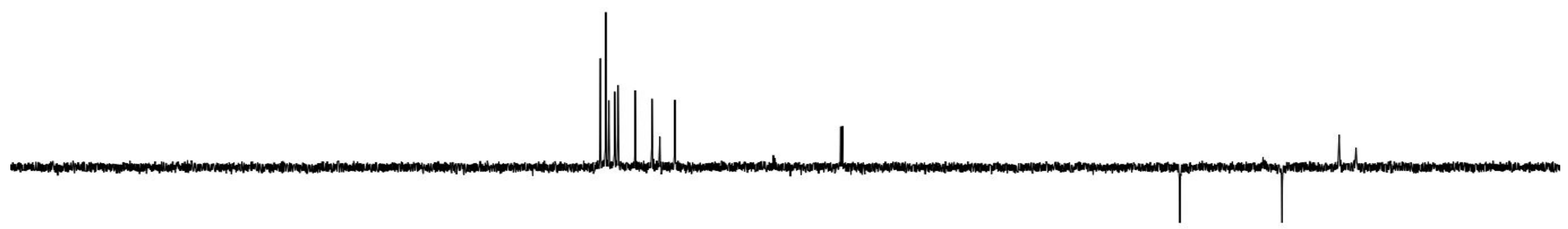

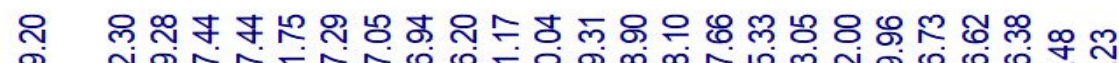

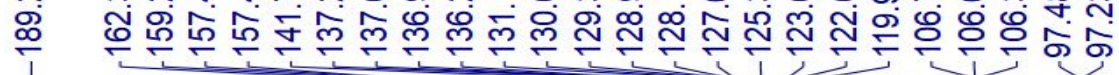

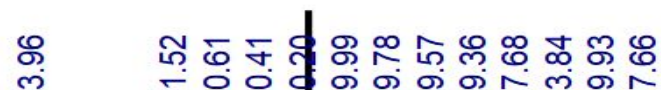

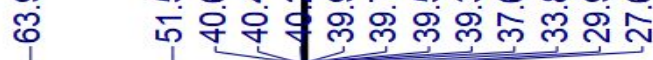

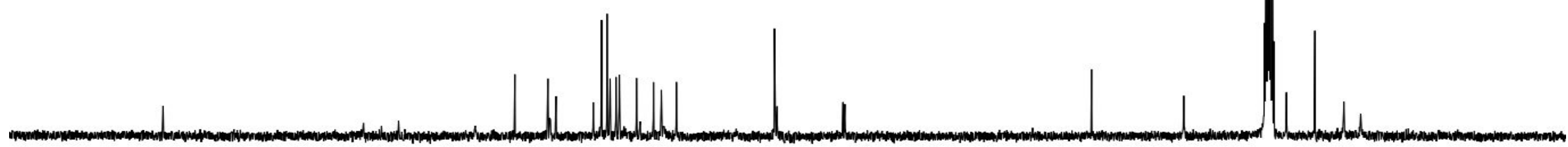

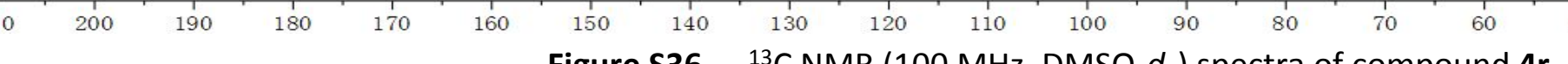

Figure S36. ${ }^{13} \mathrm{C}$ NMR $\left(100 \mathrm{MHz}\right.$, DMSO- $\left.d_{6}\right)$ spectra of compound $\mathbf{4 r}$ 

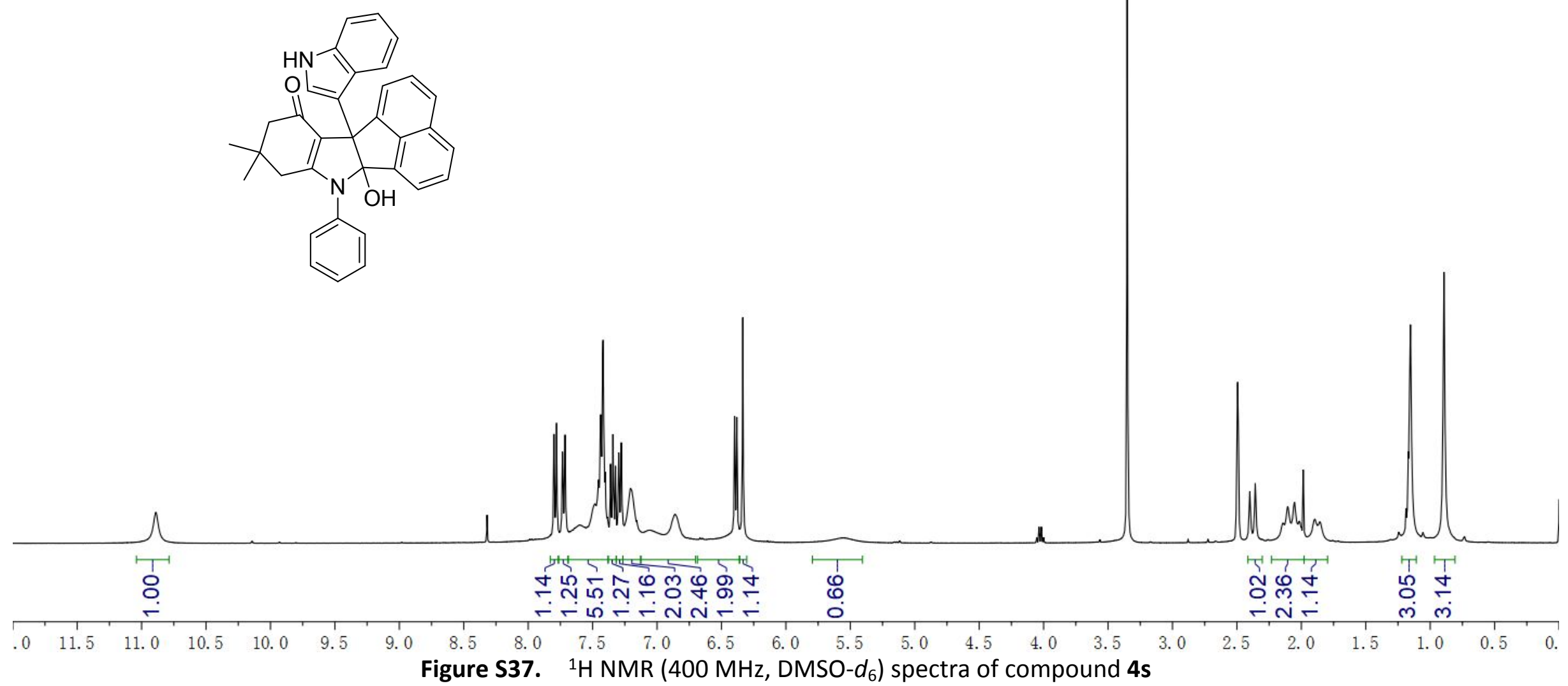

Figure S37. ${ }^{1} \mathrm{H}$ NMR $\left(400 \mathrm{MHz}, \mathrm{DMSO}-d_{6}\right)$ spectra of compound $4 \mathrm{~s}$ 


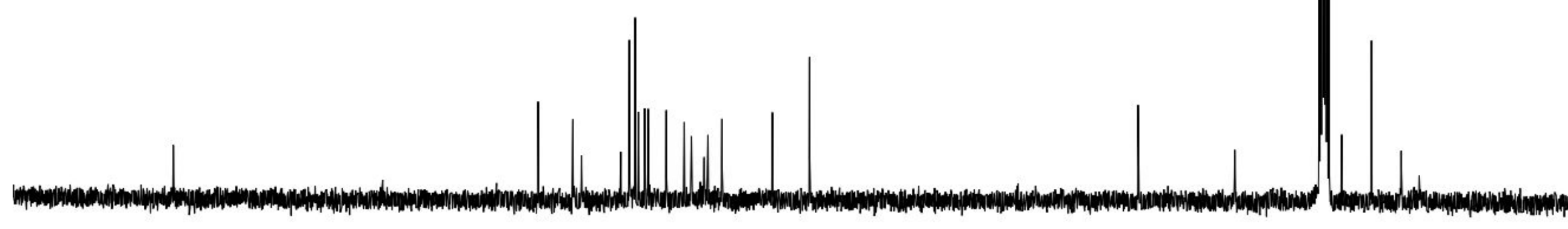




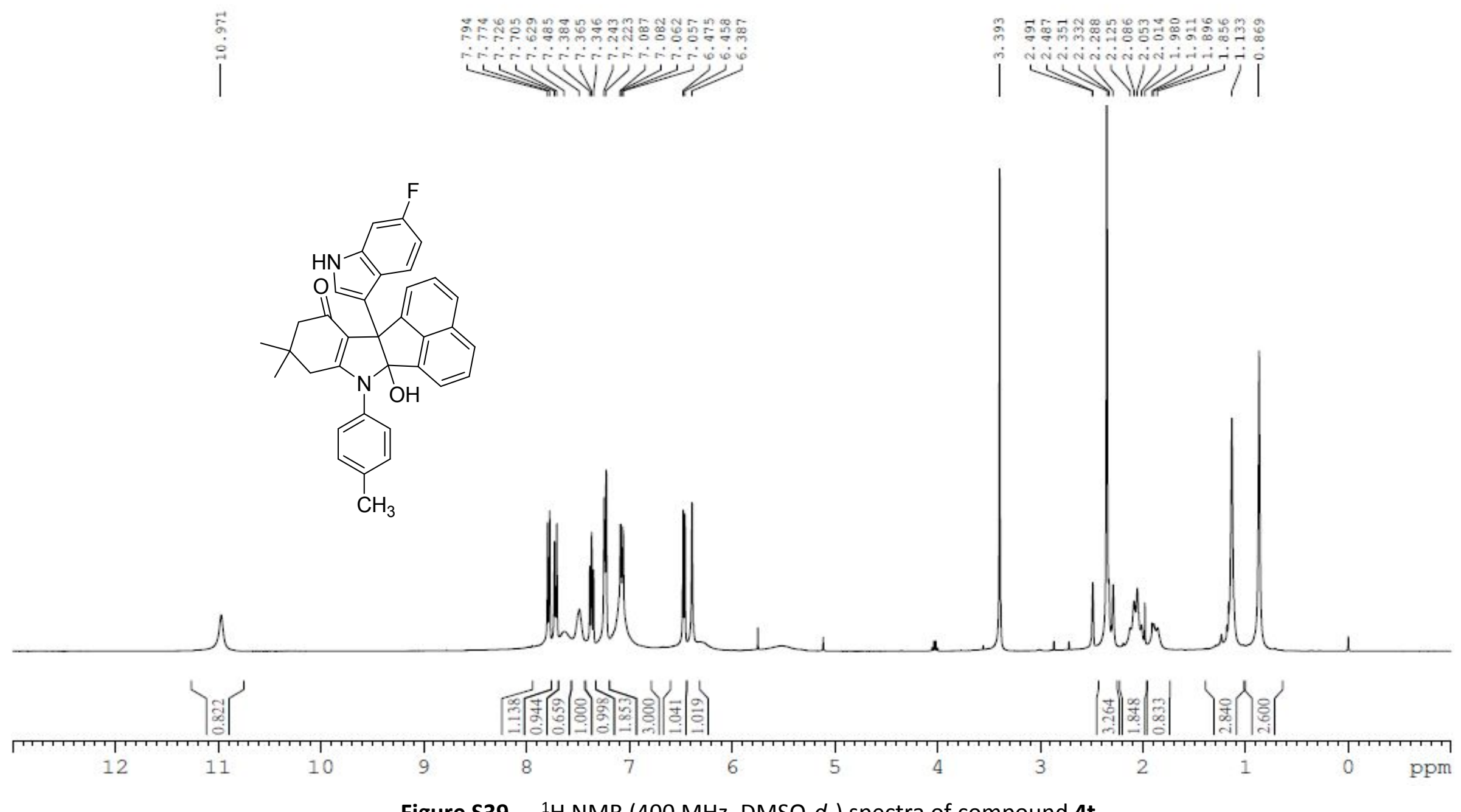

Figure S39. ${ }^{1} \mathrm{H}$ NMR (400 MHz, DMSO- $\left.d_{6}\right)$ spectra of compound $\mathbf{4 t}$ 


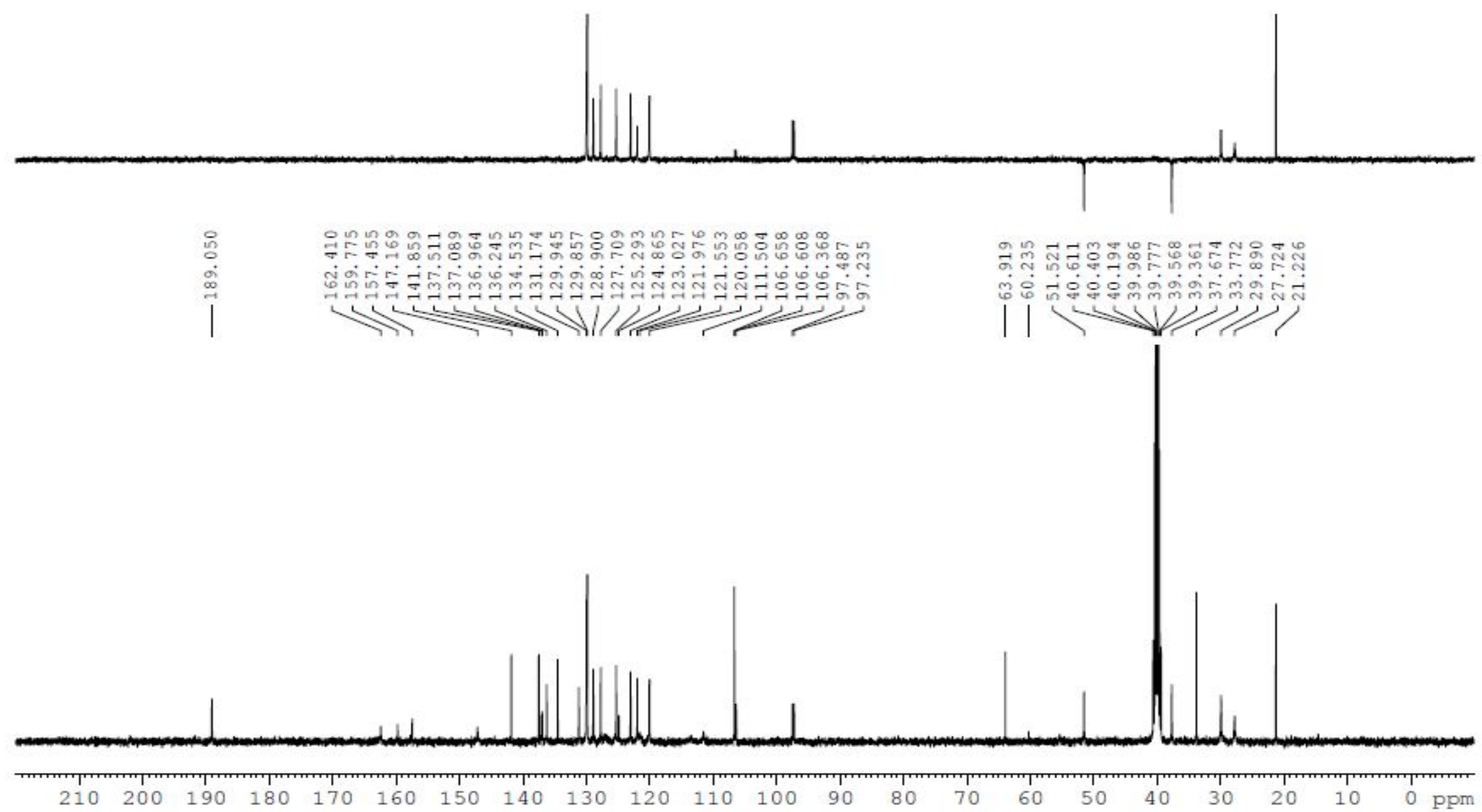

Figure S40. ${ }^{13} \mathrm{C}$ NMR (100 MHz, DMSO- $\left.d_{6}\right)$ spectra of compound 4t 


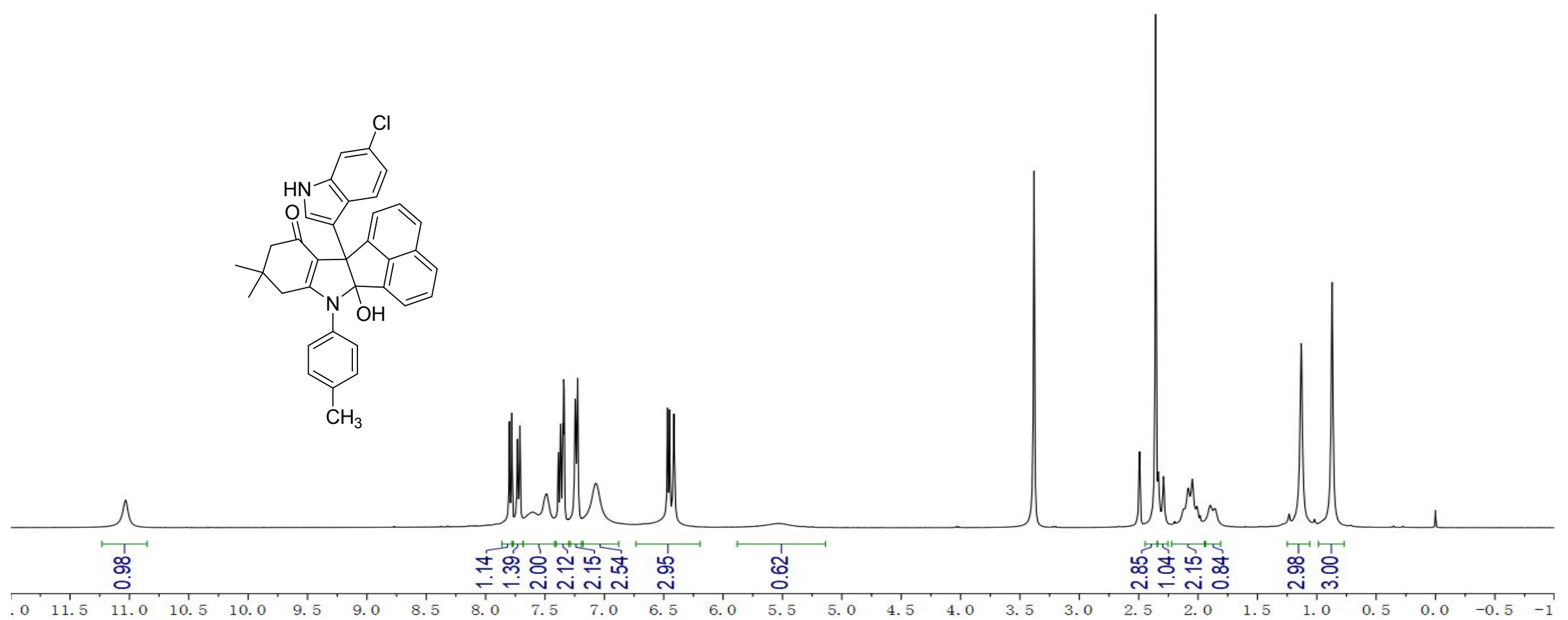

Figure S41. ${ }^{1} \mathrm{H}$ NMR (400 MHz, DMSO- $\left.d_{6}\right)$ spectra of compound $4 \mathbf{u}$ 


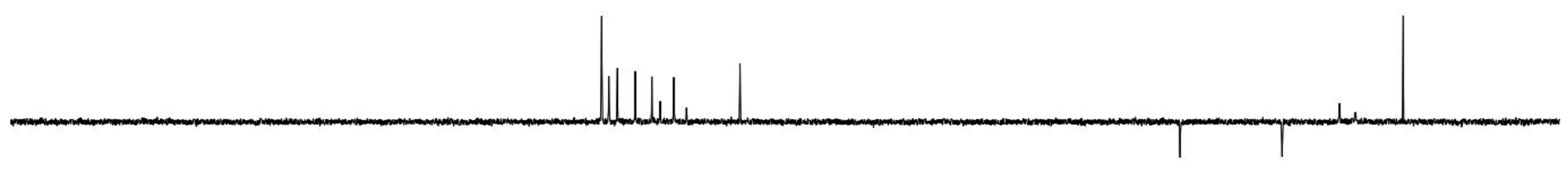

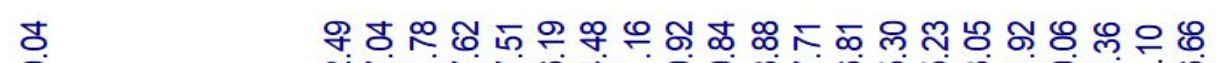

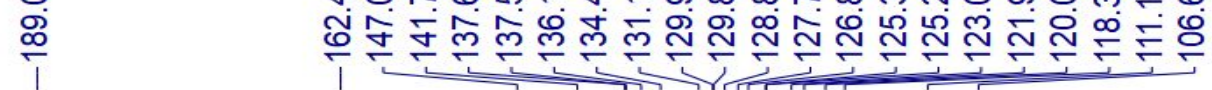

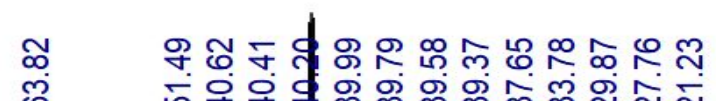

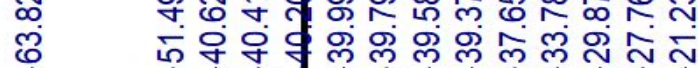

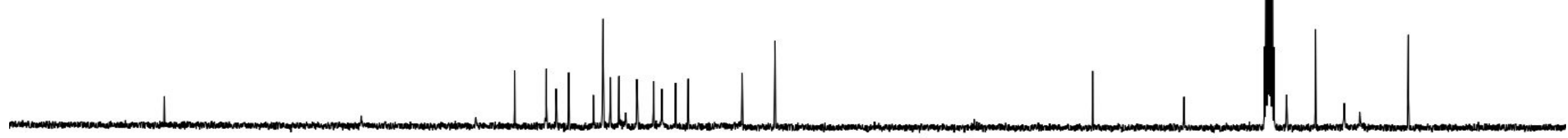

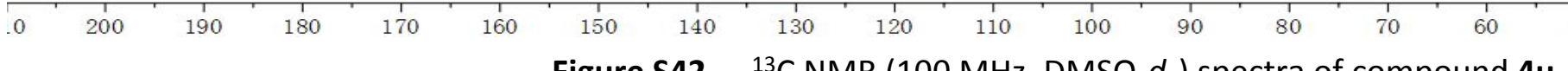

Figure S42. ${ }^{13} \mathrm{C}$ NMR $\left(100 \mathrm{MHz}, \mathrm{DMSO}-d_{6}\right)$ spectra of compound $4 \mathrm{u}$ 


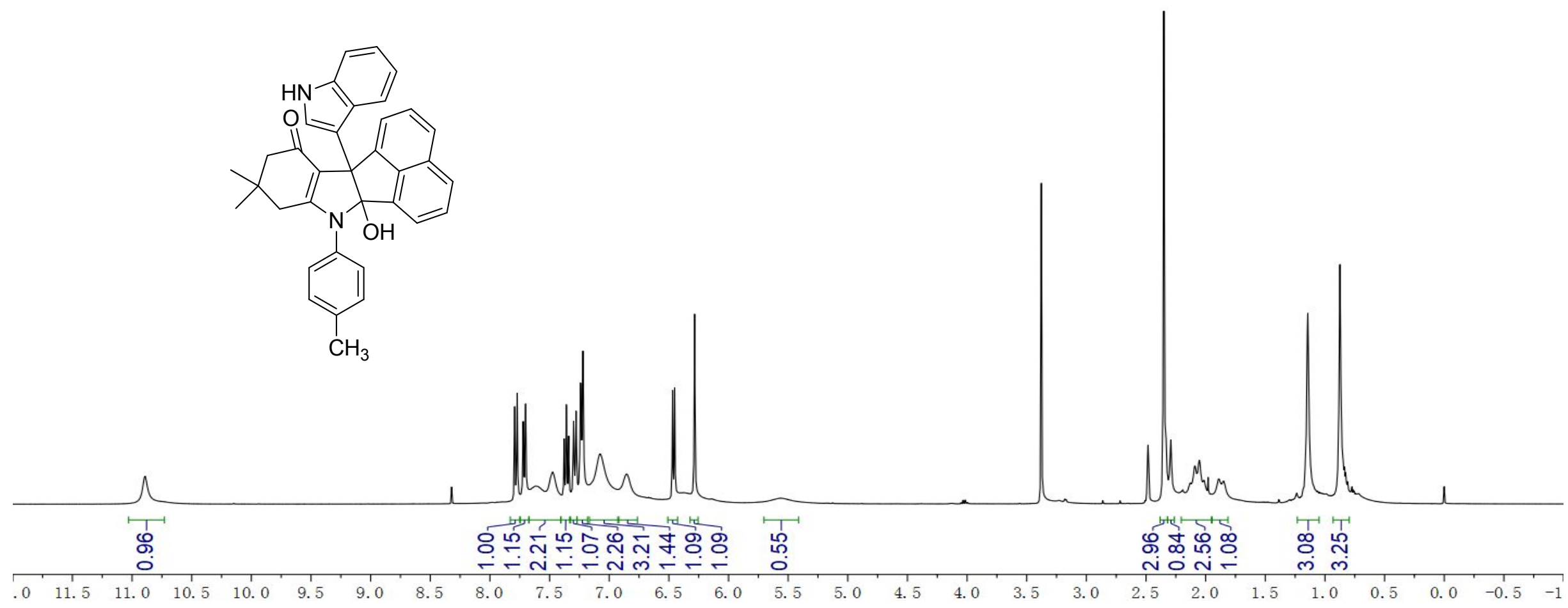

Figure S43. ${ }^{1} \mathrm{H}$ NMR $\left(400 \mathrm{MHz}, \mathrm{DMSO}-d_{6}\right)$ spectra of compound $4 \mathbf{v}$ 


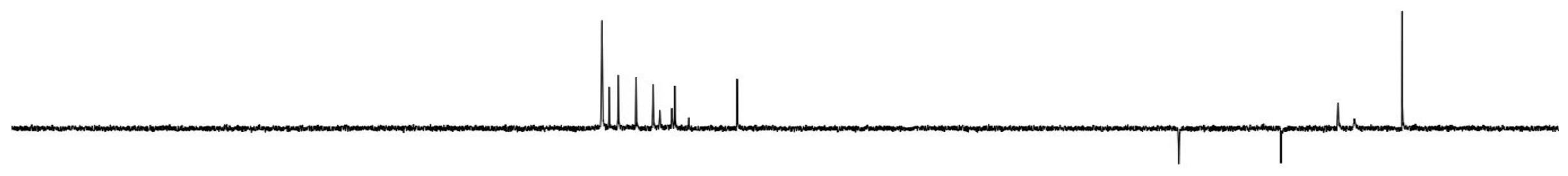

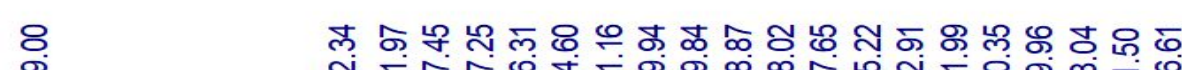

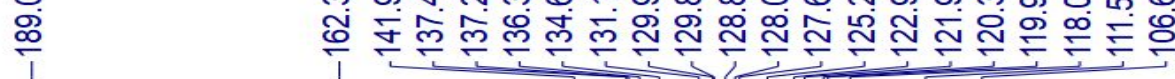

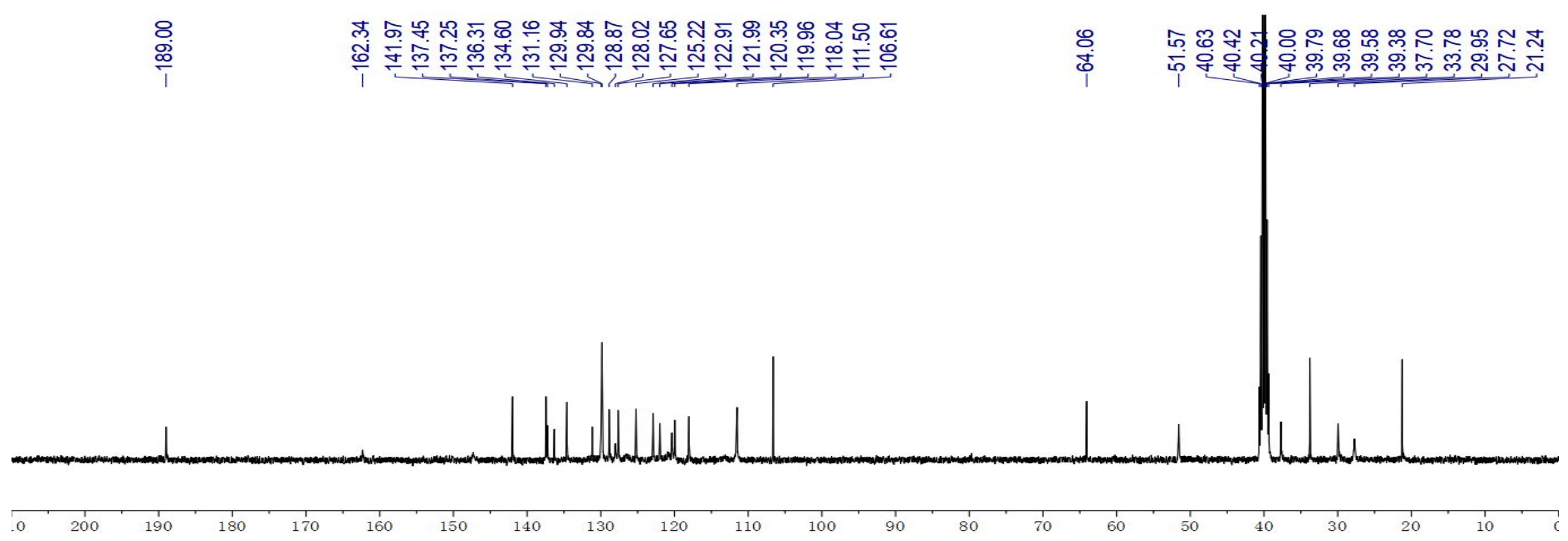

Figure S44. ${ }^{13} \mathrm{C}$ NMR (100 MHz, DMSO- $\left.d_{6}\right)$ spectra of compound $4 \mathbf{v}$ 


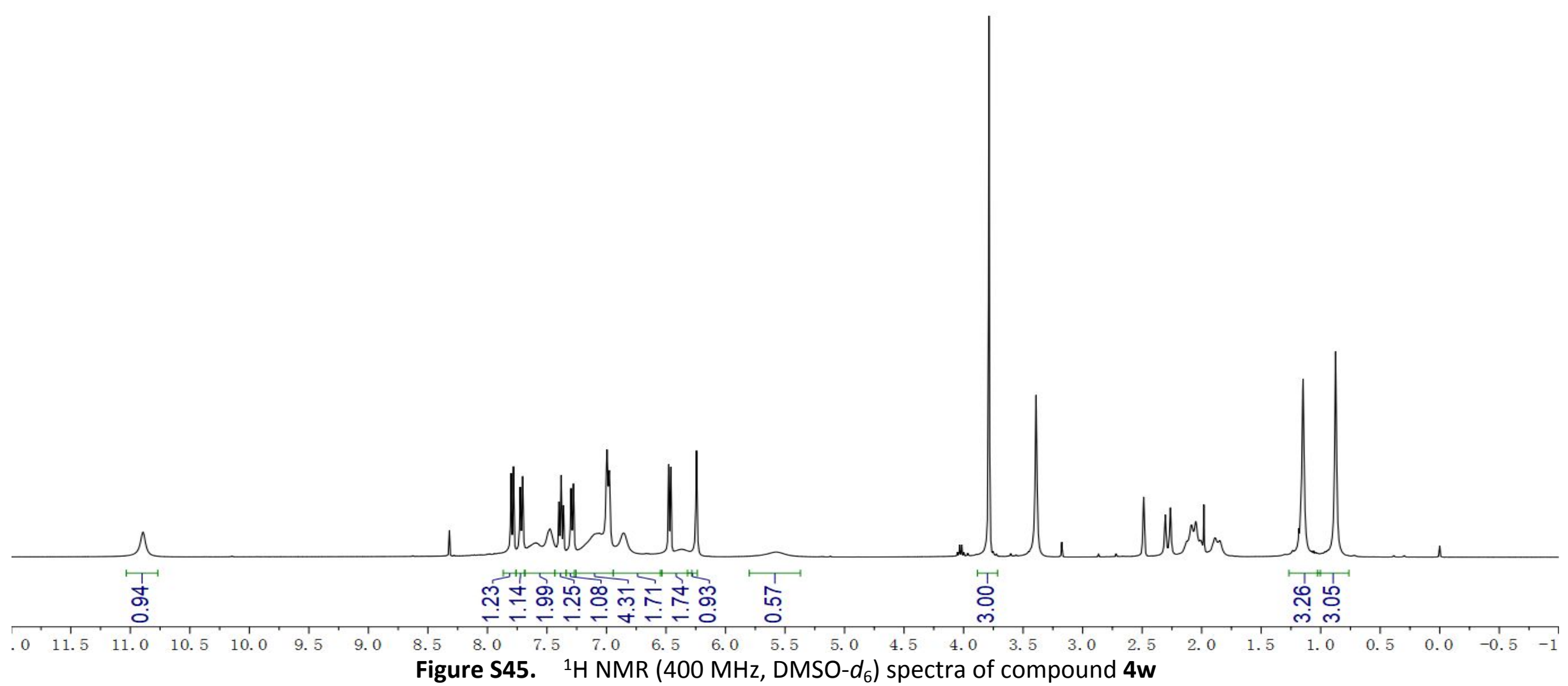



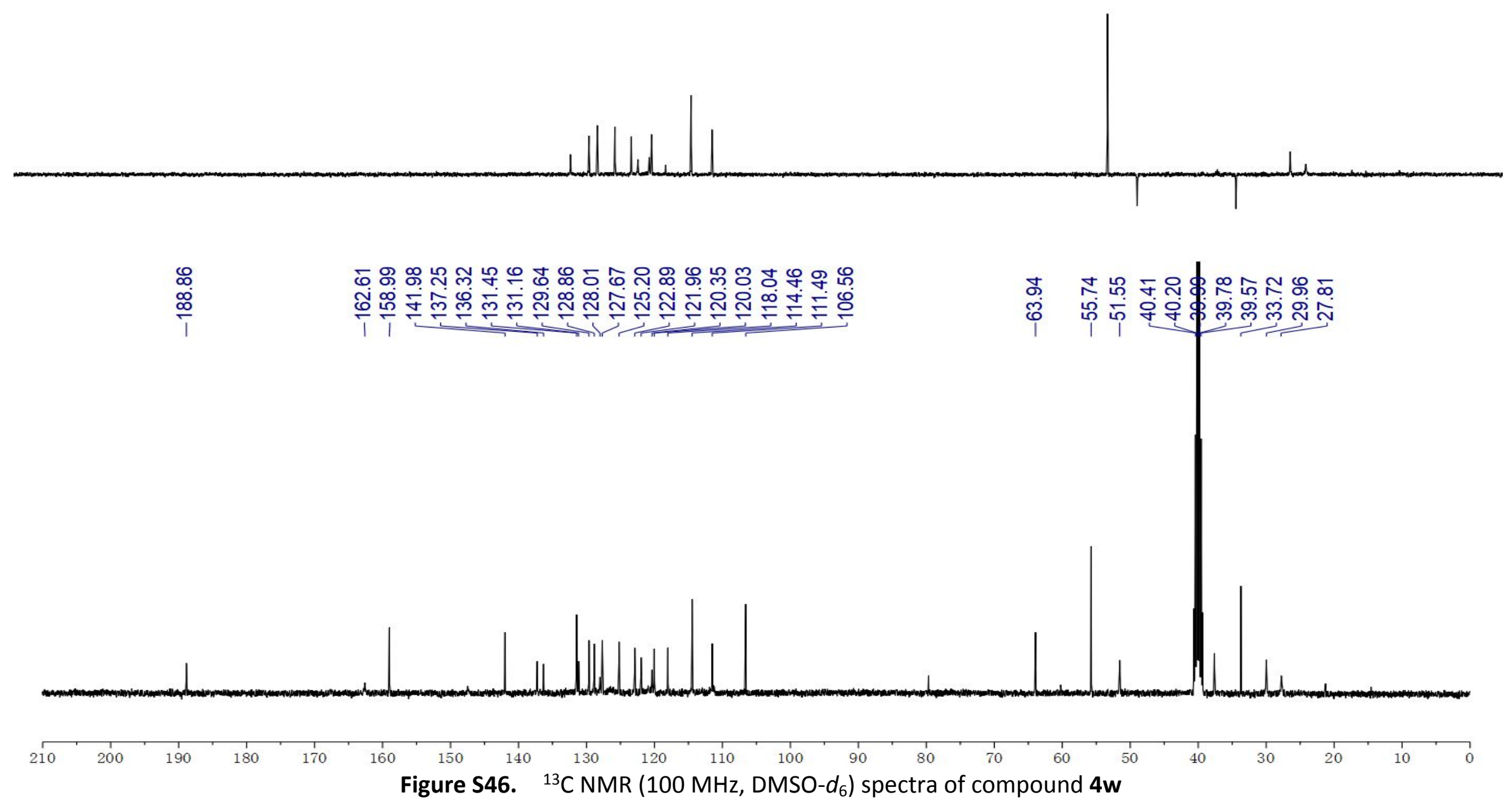


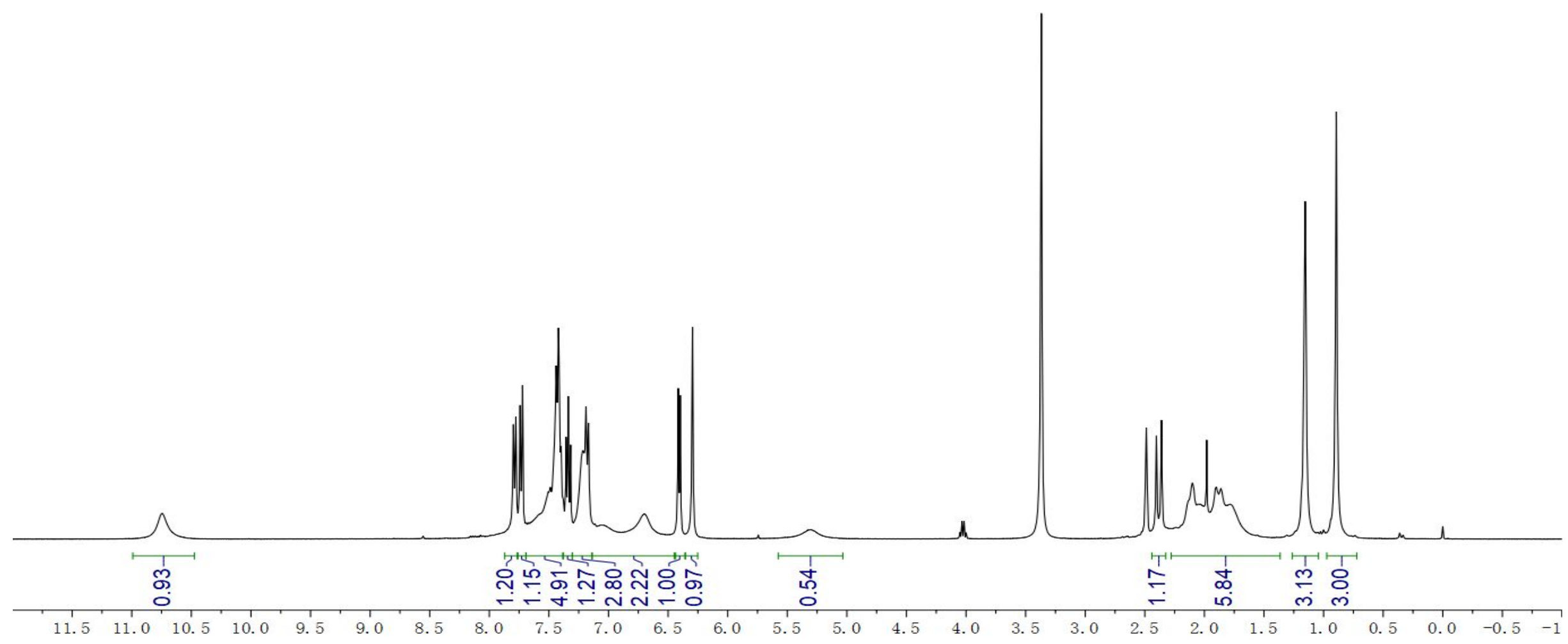

Figure S47. $\left.{ }^{1} \mathrm{H} \mathrm{NMR} \mathrm{(400} \mathrm{MHz,} \mathrm{DMSO-} d_{6}\right)$ spectra of compound $\mathbf{4 x}$ 


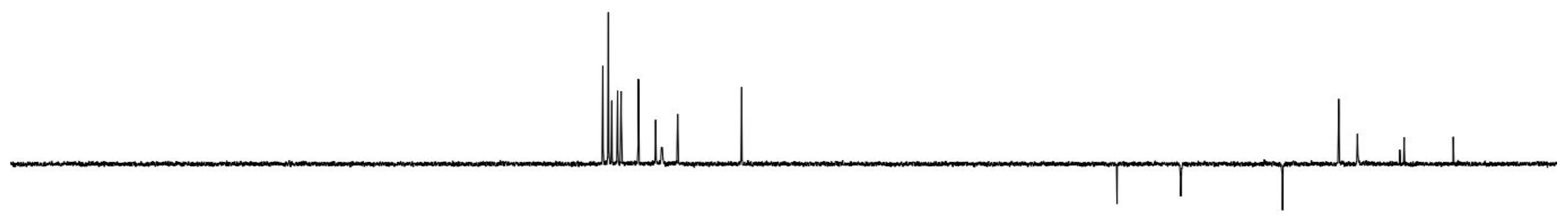

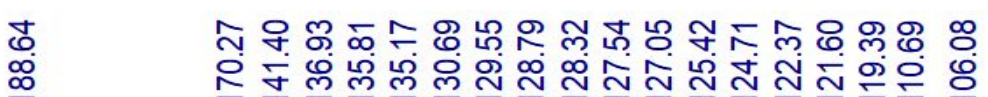

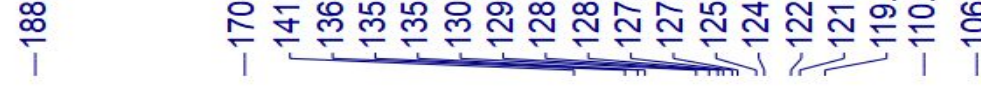

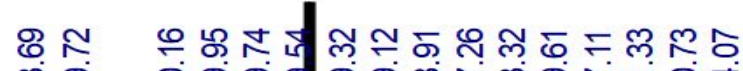

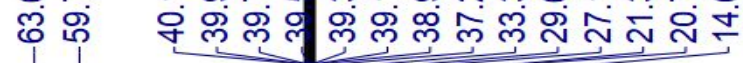

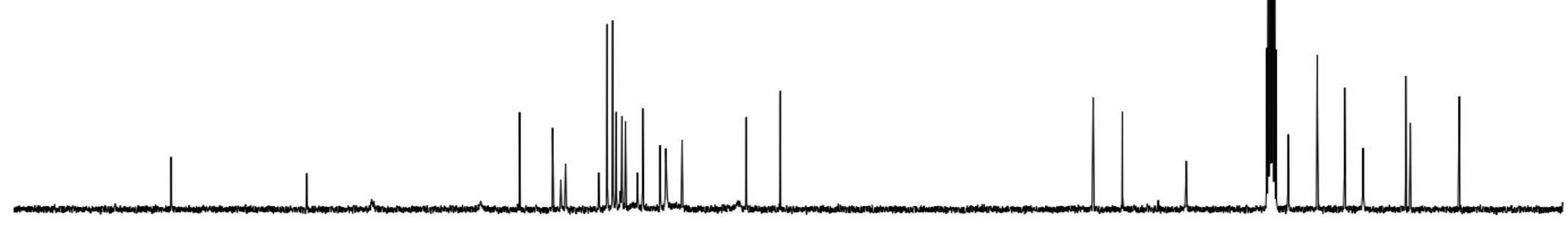

Figure S48. ${ }^{13} \mathrm{C}$ NMR $\left(100 \mathrm{MHz}, \mathrm{DMSO}-d_{6}\right)$ spectra of compound $\mathbf{4 x}$ 


\section{$\underline{{ }^{1} \mathrm{H} \text { NMR and }{ }^{13} \mathrm{C} \text { NMR spectra for intermediate } 5 \text { and } 6}$}

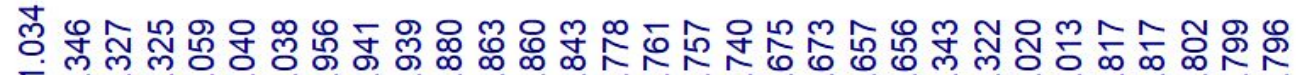

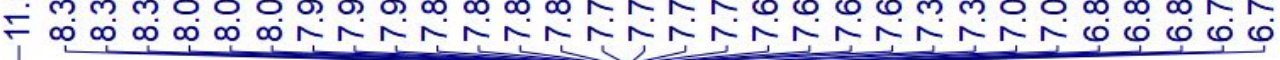

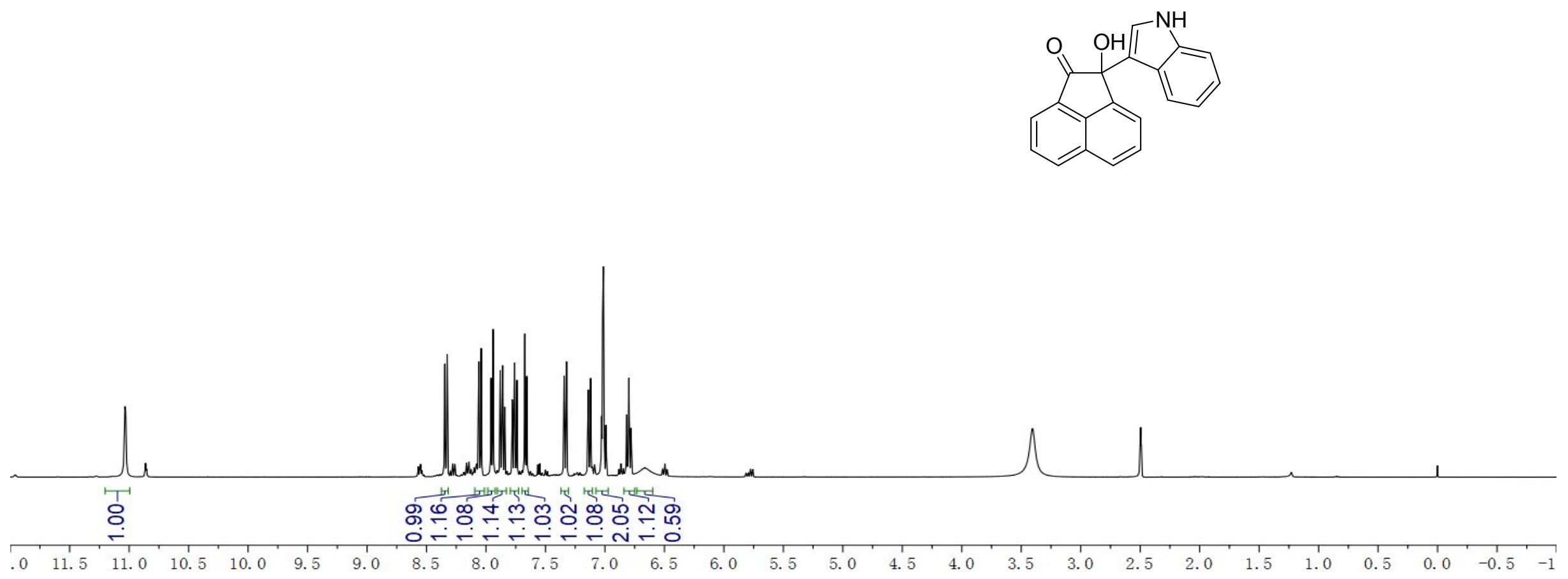

Figure S49. ${ }^{1} \mathrm{H}$ NMR (400 MHz, DMSO- $\left.d_{6}\right)$ spectra of compound $\mathbf{5}$ 


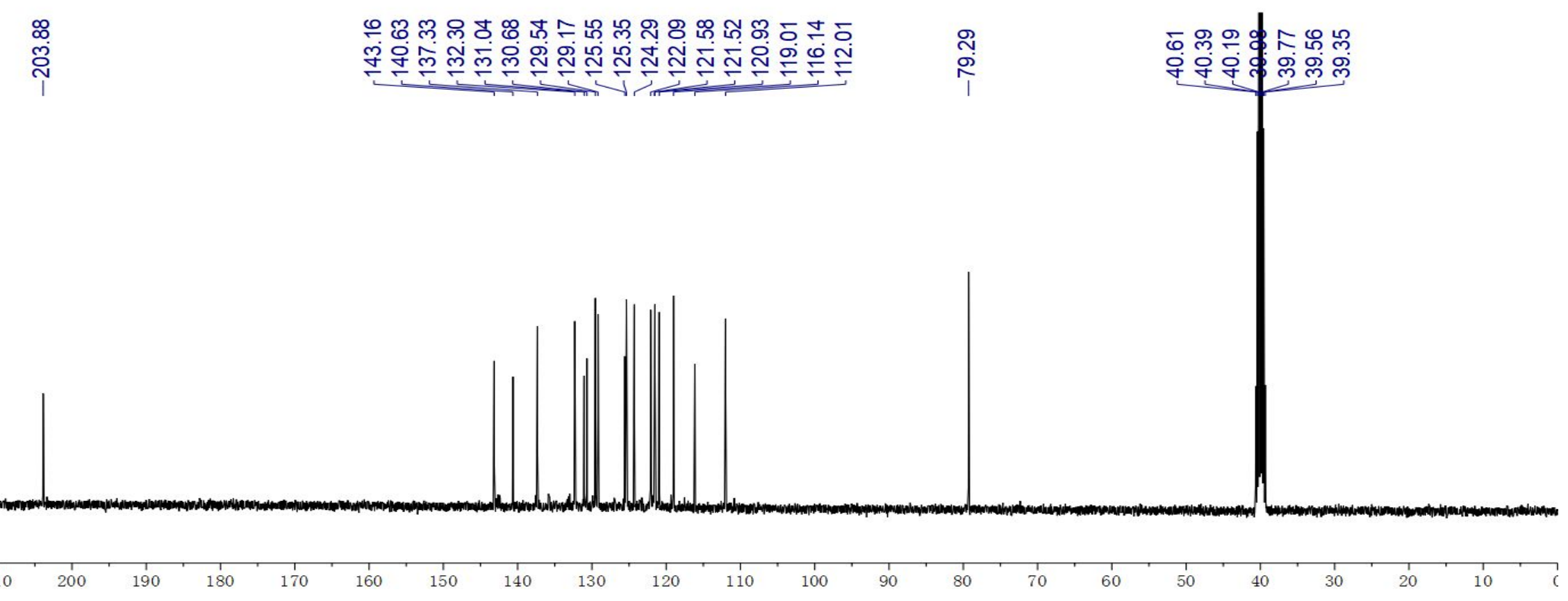

Figure S50. ${ }^{13} \mathrm{C}$ NMR $\left(100 \mathrm{MHz}, \mathrm{DMSO}-d_{6}\right)$ spectra of compound 5 


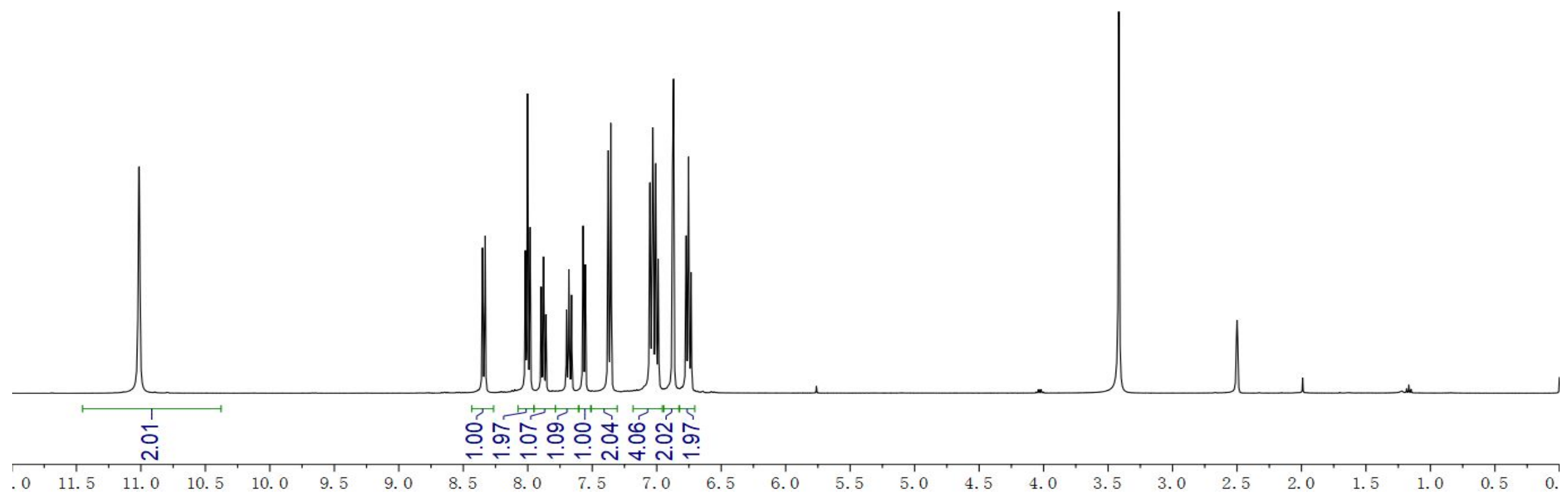

Figure S51. ${ }^{1} \mathrm{H}$ NMR $\left(400 \mathrm{MHz}, \mathrm{DMSO}-d_{6}\right)$ spectra of compound 6 


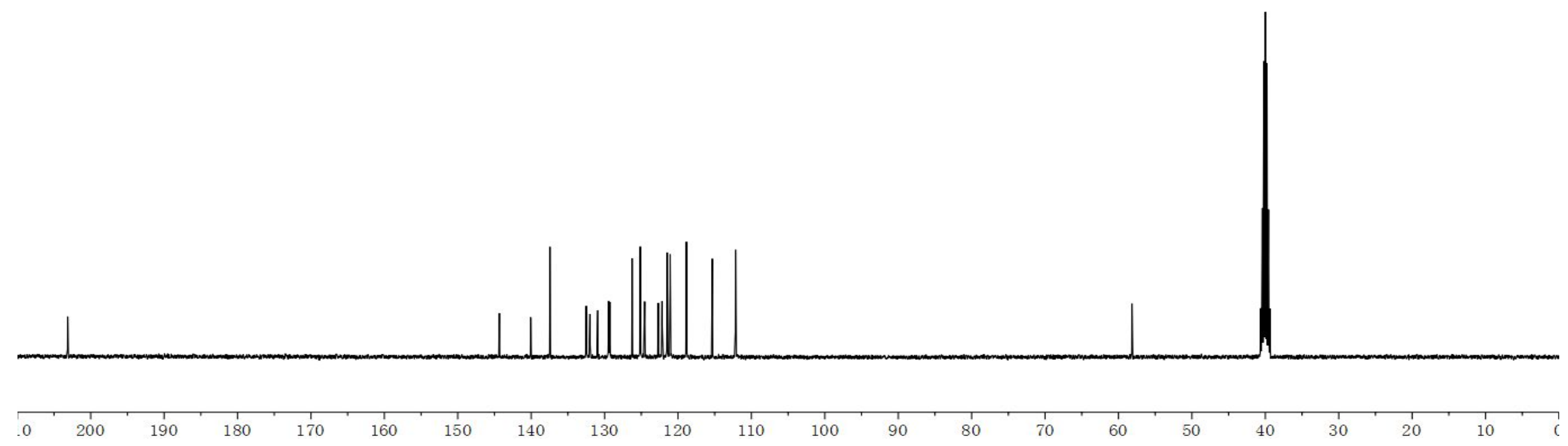

Figure S52. ${ }^{13} \mathrm{C}$ NMR $\left(100 \mathrm{MHz}, \mathrm{DMSO}-d_{6}\right)$ spectra of compound 6 


\section{HMRS spectra for representative compound 4}

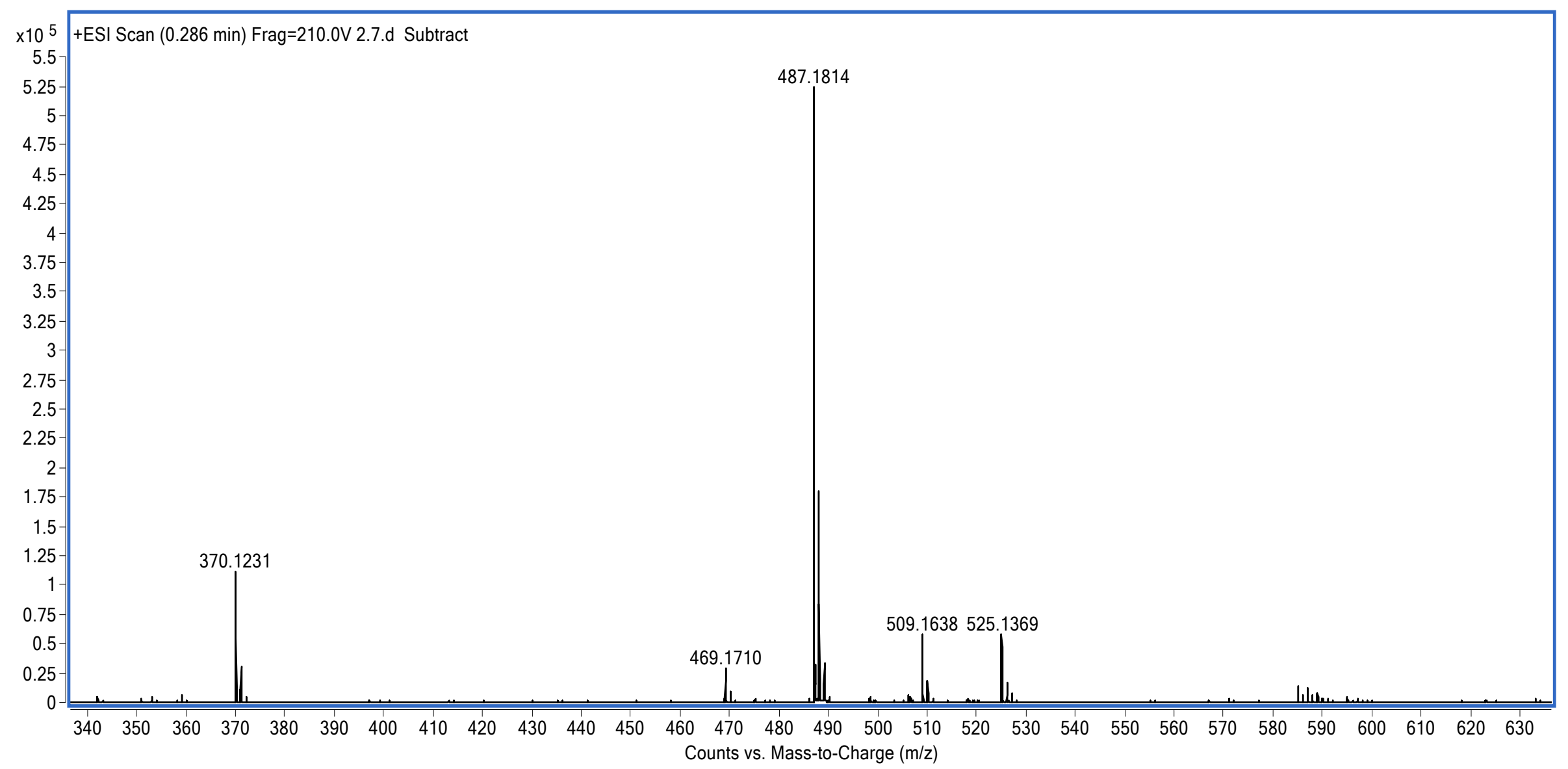

Figure S53. HRMS spectra of compound 4a 


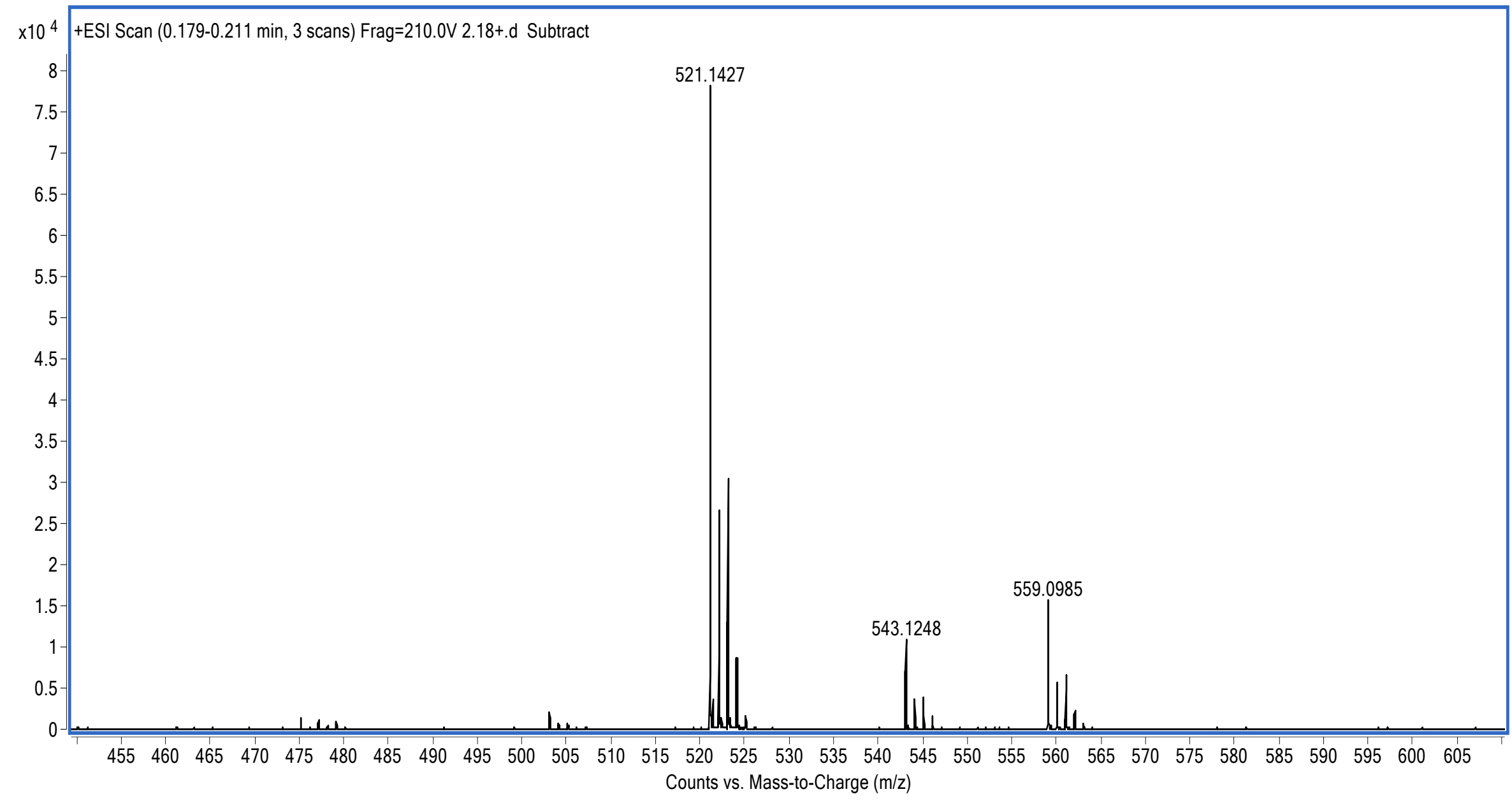

Figure S54. HRMS spectra of compound $\mathbf{4 b}$ 


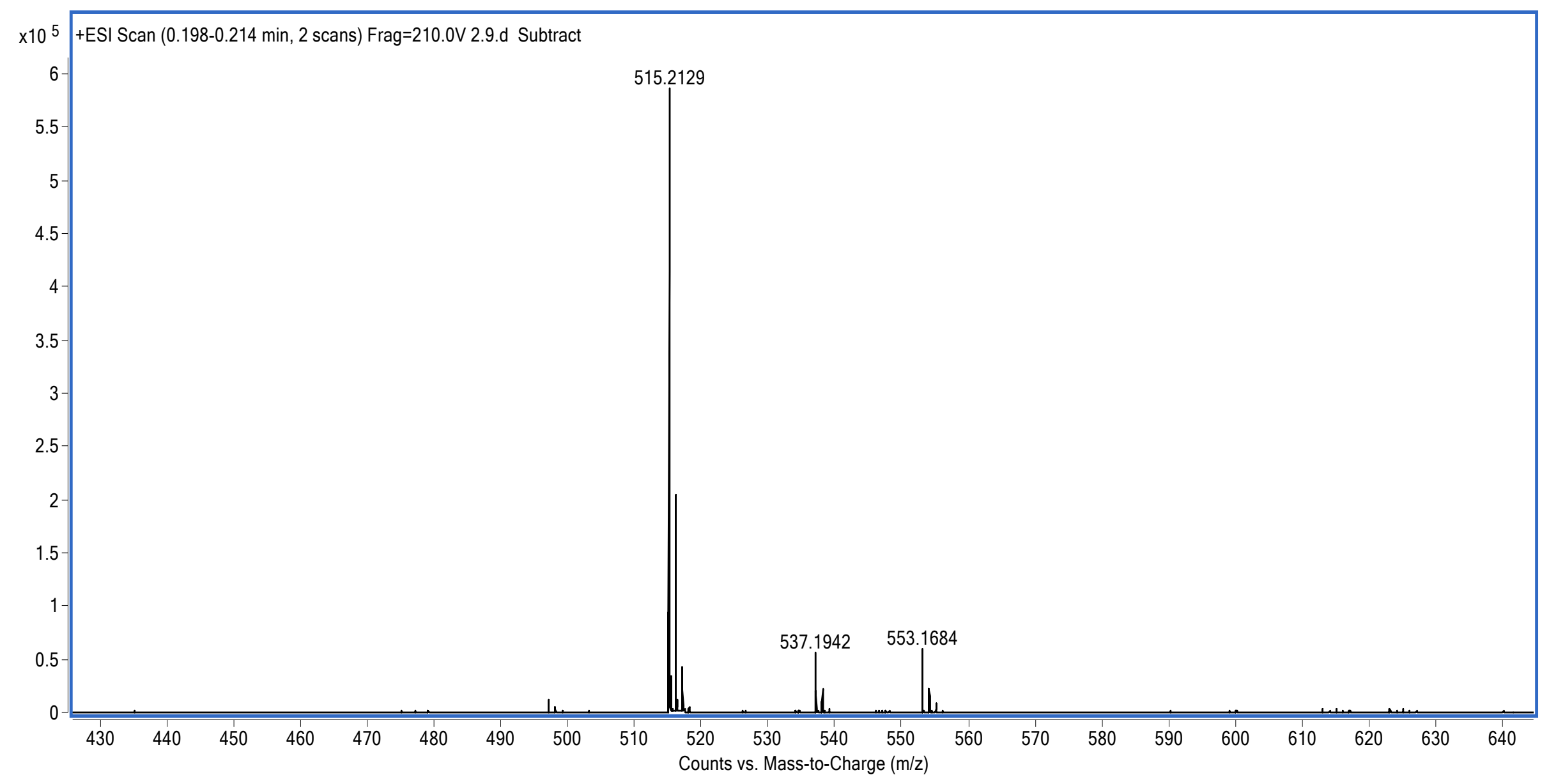

Figure S55. HRMS spectra of compound $4 \mathrm{~m}$ 


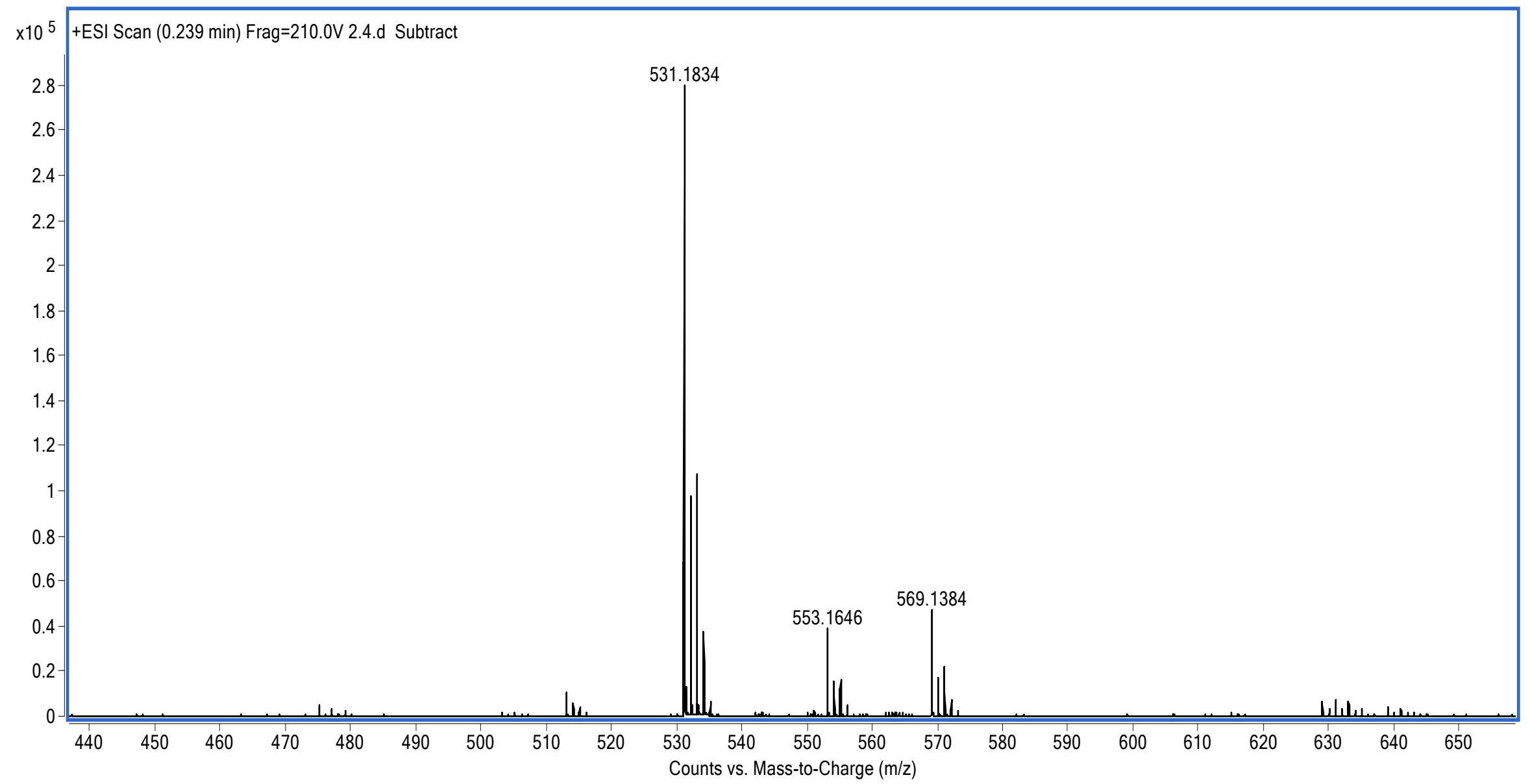

Figure S56. HRMS spectra of compound $4 p$ 


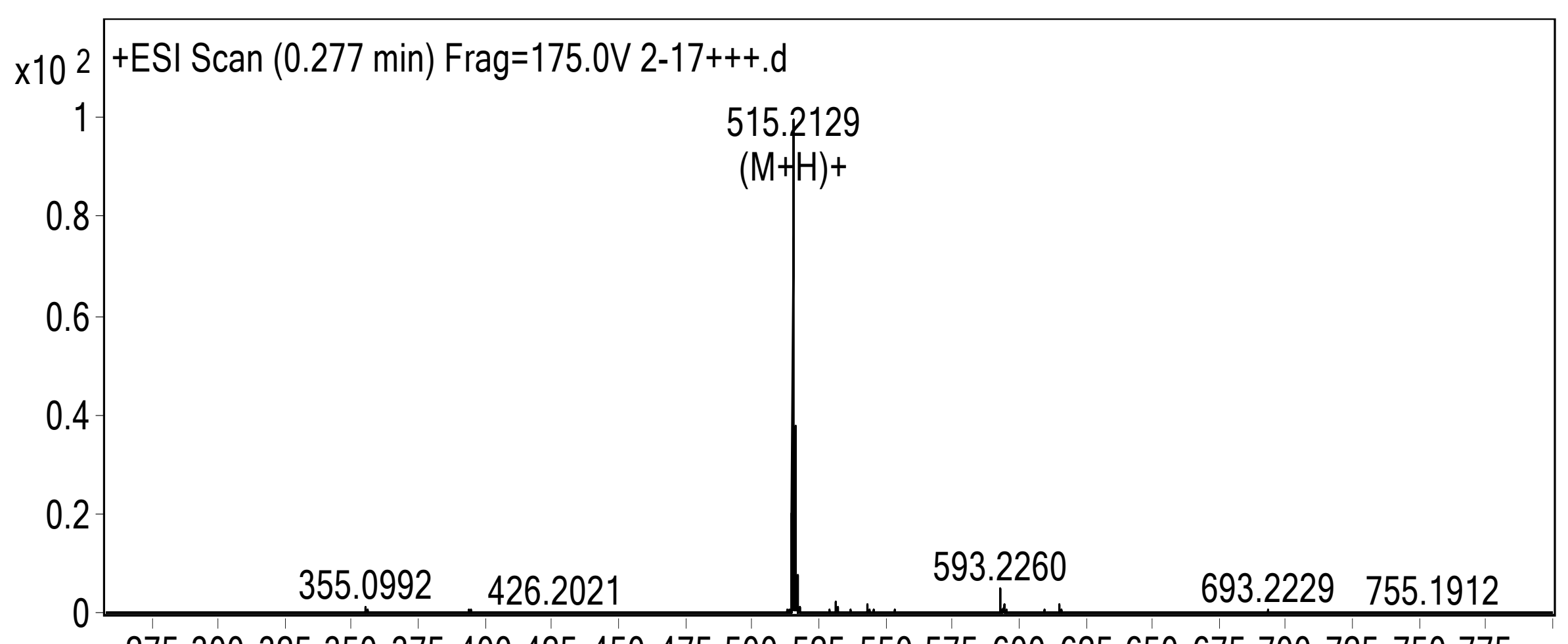

275300325350375400425450475500525550575600625650675700725750775 Counts (\%) vs. Mass-to-Charge $(\mathrm{m} / \mathrm{z})$

Figure S57. HRMS spectra of compound $\mathbf{4 r}$ 


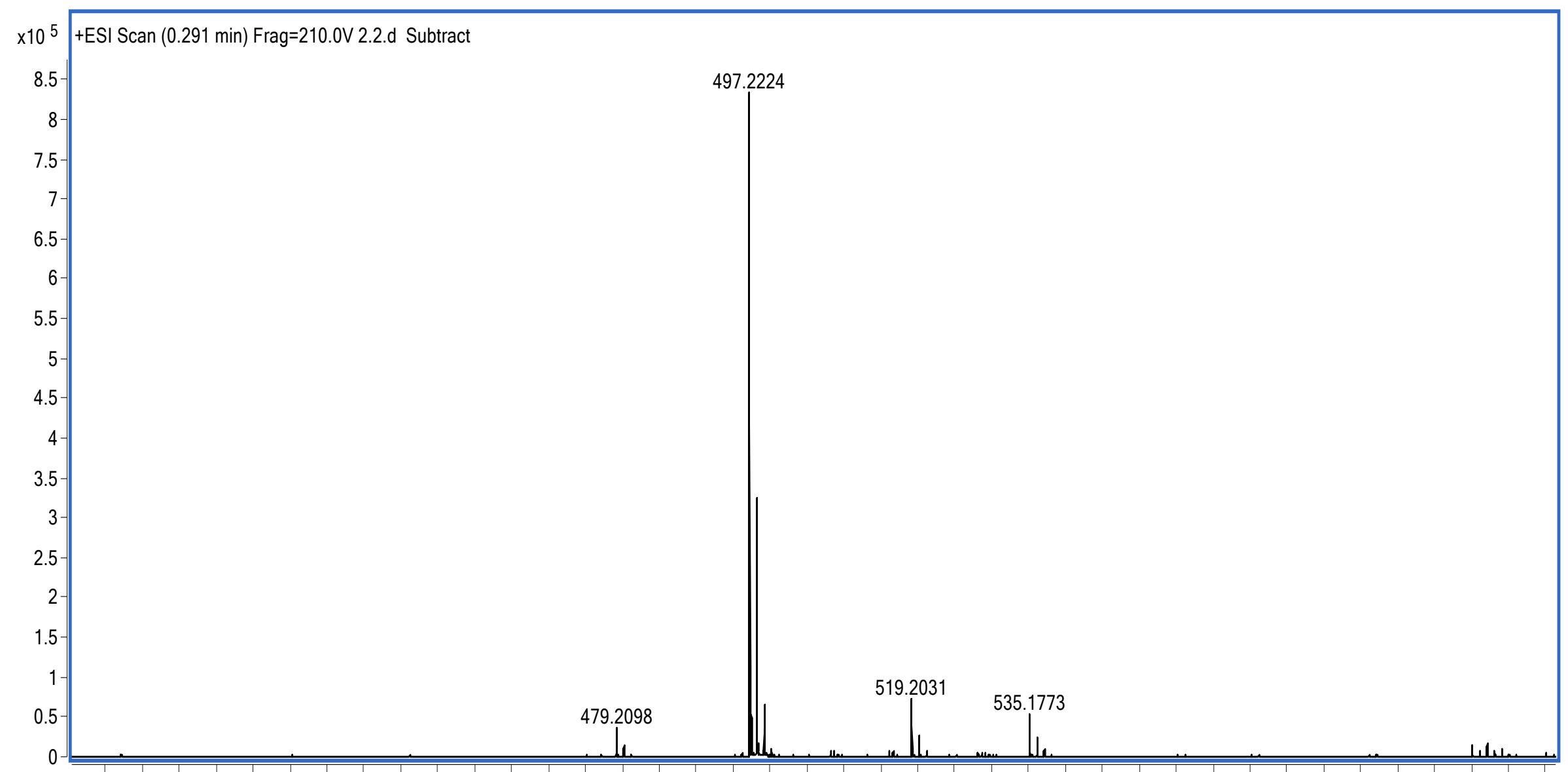

410415420425430435440445450455460465470475480485490495500505510515520525530535540545550555560565570575580585590595600 Counts vs. Mass-to-Charge $(\mathrm{m} / \mathrm{z})$

Figure S58. HRMS spectra of compound 4s 


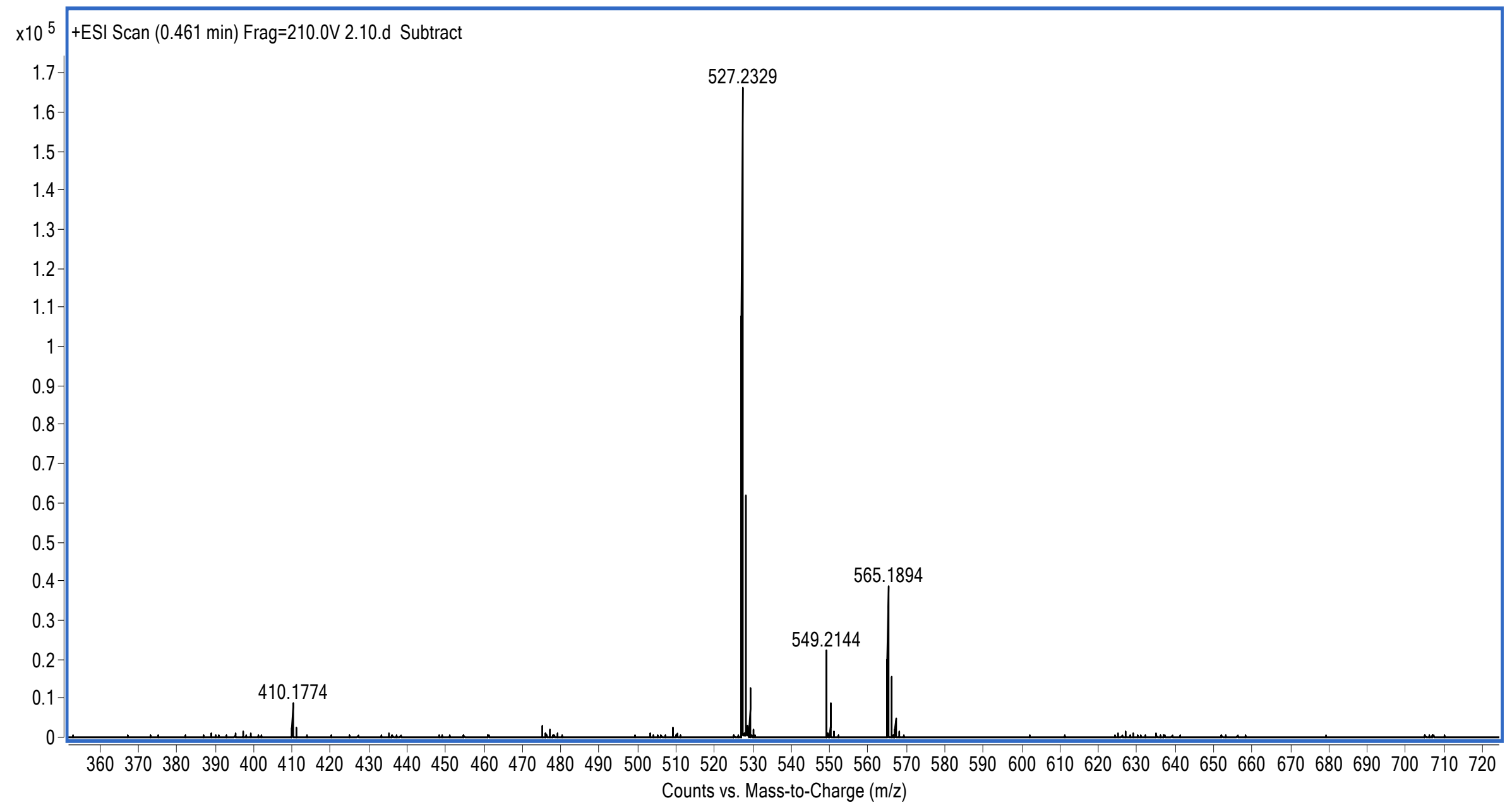

Figure S59. HRMS spectra of compound $\mathbf{4 w}$ 